\title{
Deep Aesthetic Assessment of Breast Cancer Surgery Outcomes
}

Tiago Filipe Sousa Gonçalves

MASTER's THESIS

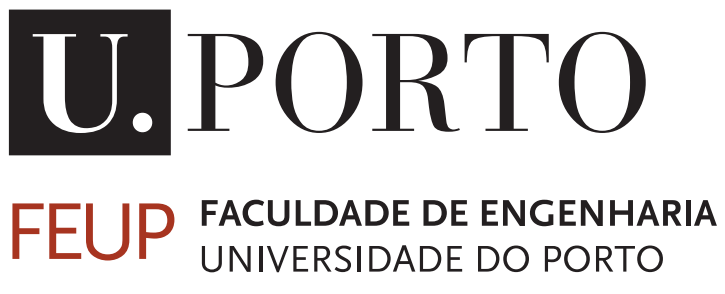

Integrated Master in Bioengineering

Supervisor: Jaime dos Santos Cardoso, $\mathrm{PhD}$

Second Supervisor: Wilson José dos Santos Silva, MSc

July 21, 2019 
(C) Tiago Filipe Sousa Gonçalves, 2019 


\title{
Deep Aesthetic Assessment of Breast Cancer Surgery Outcomes
}

\author{
Tiago Filipe Sousa Gonçalves
}

Integrated Master in Bioengineering

July 21, 2019 



\section{Resumo}

O cancro da mama é uma doença altamente mutável e de rápida evolução, com grande incidência mundial. Ainda assim, estima-se que aproximadamente $90 \%$ dos casos são tratáveis e curáveis, desde que detetados em estádio precoce e se receberem o melhor tratamento. Atualmente, com a existência de hábitos de rotina no que toca ao rastreio do cancro da mama, melhores planos de tratamento clínico e uma gestão adequada da doença, é possível tratar a maioria dos cancros, recorrendo a abordagens conservativas, também conhecidas como tratamentos conservativos do cancro da mama (BCCT). Com esta metodologia de tratamento, é possível focar nos resultados estéticos da cirurgia e na qualidade de vida do paciente, o que pode influenciar as consequências do BCCT. Antigamente, esta avaliação seria feita através de métodos subjetivos, em que um painel de especialistas seria necessário para realizar a avaliação; entretanto, com o desenvolvimento de técnicas de visão computacional, métodos objetivos, como o BAT ${ }^{\circledR}$ e o BCCT.core, que realizam a avaliação tendo em conta medições relacionadas com a assimetria, têm sido utilizados. Por outro lado, eles ainda exigem informações fornecidas pelo utilizador e nenhum deles foi considerado o método de eleição para esta tarefa. Recentemente, com o advento das técnicas de deep learning, algoritmos capazes de melhorar o desempenho de métodos tradicionais na deteção de pontos fiduciais (necessários para as medições de assimetria) foram propostos e mostraram resultados promissores. Há, no entanto, uma grande margem de investigação sobre como integrar estes algoritmos numa aplicação completa, capaz de realizar uma classificação end-to-end dos resultados do BCCT. Tendo isto em conta, esta tese mostra um estudo comparativo entre deep convolutional networks para segmentação de imagens e duas arquiteturas diferentes de deteção de pontos fiduciais baseadas no conceito de qualidade para a deteção do contorno das mamas. Uma que usa um modelo de deep learning que aprendeu a prever a qualidade (dada pelo erro quadrático médio) de um vetor de pontos fiduciais e, com base nessa qualidade, aplica o algoritmo de backpropagation, através de gradient descent, para melhorá-los; outra que utiliza um modelo de deep learning que foi treinado com a qualidade como um método de regularização e que utiliza o conceito de refinamento iterativo, em cada etapa do treino, para melhorar a qualidade dos pontos fiduciais que foram introduzidos na rede. Embora nenhum dos métodos ultrapasse o método do estado da arte, eles apresentam resultados promissores para a criação de metodologias alternativas para a resolução de outros problemas de regressão, em que a aprendizagem da métrica de qualidade poderá ser mais fácil. Acompanhando a tendência atual na área do desenvolvimento web e com o objetivo de transferir o BCCT.core para um formato online, foi desenvolvido um protótipo de uma aplicação web para a deteção automática de pontos fiduciais, que é apresentado neste documento. Atualmente, o utilizador pode enviar uma imagem, detetar automaticamente os seus pontos fiduciais e calcular o valor do Breast Retraction Assessment (BRA). Este protótipo é completamente escalável e pode ser atualizado com novas funcionalidades de acordo com as necessidades do utilizador. 


\section{Abstract}

Breast cancer is a highly mutable and rapidly evolving disease, with a large worldwide incidence. Even though, it is estimated that approximately $90 \%$ of the cases are treatable and curable if detected on early staging and given the best treatment. Nowadays, with the existence of breast cancer routine screening habits, better clinical treatment plans and proper management of the disease, it is possible to treat most cancers with conservative approaches, also known as breast cancer conservative treatments (BCCT). With such a treatment methodology, it is possible to focus on the aesthetic results of the surgery and the patient's Quality of Life, which may influence BCCT outcomes. In the past, this assessment would be done through subjective methods, where a panel of experts would be needed to perform the assessment; however, with the development of computer vision techniques, objective methods, such as BAT ${ }^{\complement}$ and BCCT.core, which perform the assessment based on asymmetry measurements, have been used. On the other hand, they still require information given by the user and none of them has been considered the gold standard for this task. Recently, with the advent of deep learning techniques, algorithms capable of improving the performance of traditional methods on the detection of breast fiducial points (required for asymmetry measurements) have been proposed and showed promising results. There is still, however, a large margin for investigation on how to integrate such algorithms in a complete application, capable of performing an end-to-end classification of the BCCT outcomes. Taking this into account, this thesis shows a comparative study between deep convolutional networks for image segmentation and two different quality-driven keypoint detection architectures for the detection of the breast contour. One that uses a deep learning model that has learned to predict the quality (given by the mean squared error) of an array of keypoints, and, based on this quality, applies the backpropagation algorithm, with gradient descent, to improve them; another which uses a deep learning model which was trained with the quality as a regularization method and that used iterative refinement, in each training step, to improve the quality of the keypoints that were fed into the network. Although none of the methods surpasses the current state of the art, they present promising results for the creation of alternative methodologies to address other regression problems in which the learning of the quality metric may be easier. Following the current trend in the field of web development and with the objective of transferring BCCT.core to an online format, a prototype of a web application for the automatic keypoint detection was developed and is presented in this document. Currently, the user may upload an image, automatically detect and/or manipulate its keypoints and compute the Breast Retraction Assessment (BRA) value. This prototype is completely scalable and can be upgraded with new functionalities according to the user's needs. 


\section{Acknowledgements}

This work would not be possible without the help and presence of the outstanding people that contributed to all the history that made my Academic life in the past six years. I promise that all the efforts were made in order to remember all of you.

First of all, I would like to thank Professor Jaime Cardoso for all the proposed challenges, support and good ideas. In this past year, I really developed my skills and my passion for this research topic grew even wider.

To Wilson Silva, who was always there from the very beginning to guide me through this journey. Thank you for all the brainstorming, all the motivating words and all the time that you invested in this thesis.

To my family, especially my mother, father and sister, for being my best friends, for never giving up on me and for supporting me, even in the darkest times; thank you. I would not be the man that I am today if you did not make efforts on providing me with the best educational and personal opportunities and experiences that I could have. I hope to have the time to return you everything, one day.

To all the Engineering students that I had the pleasure to meet: thank you for the friendship and the funny moments. Allow me, however, to nominate some special people that had a big impact on the person that I am today: Paulo Maia, Vitor Minhoto, Gabriel Lopes, Marina Castro, Diogo Martins, Cristiana Carpinteiro, Mariana Dias, Margarida Fernandes, Francisca Morgado, Joana Rodrigues, Carolina Avelar, Helena Ferreira, Mariana Coelho, Francisco Raio, Luís Costa, Filipa Adonias, Catarina Dias and Catarina Lourenço; thank you for all the geniality and friendship. I wish you the best!

To ShARE-UP, which I had the pleasure and the challenge to lead during this year: thank you for everything you gave me as a student, consultant and human being. To Rui Oliveira, André Morim, João Matos, Duarte Ferreira, Tiago Leal, Armando Ferreira, Alexandra Leite, João Costa, Francisco Mendes, Luiz Vasconcelos and Diogo Cunha, for being the best management team ever. To Álvaro Samagaio, which was at my side in the graduation ceremony, even though he had come directly from the United Kingdom; thank you for being there. To the younger ones, please keep improving the quality of our projects. You all deserve the best. Count on me for everything.

To NEB FEUP/ICBAS, which allowed me to participate in all the good initiatives that Bioengineering students develop for the Academic community, thank you for the work you do to help your peers. I hope to be present in the next Symposium on Bioengineering and to see that everything is going well. 
To Associação Nacional de Estudantes de Engenharia Biomédica, which I had the pleasure to co-found and to manage: the work has only begun, we have to potential to be something bigger in the next years, and I hope to see that as an alumnus. To Abel Nicolau, Ana Freire, Luís Venâncio, Rui Garcia, Dani Silva, Carlos Mendes and Juliana Guerra, for all the adventures and friendship. To all our members, thank you for the invested time in this project.

To ESSA Project, which showed me the importance of social responsibility, and how we can apply that in every detail of our lives, thank you for all the trips, reports, adventures and friends that I have made.

To Sociedade de Debates da Universidade do Porto, which trusted in me to be part of the management team in this following year, I promise to do my best!

To KIZUNA, my second family, that have accompanied me from a very young age and who, through the martial arts, taught me important values, thank you for these years of training, challenges and stories.

Last, but not least, to a very special group of people, entitled Boémios com Classe, which has been walking this path with me since high school, which has always time and patience to hear all my silly ideas and, I am sure, will change the World: thank you for almost a decade of endless nights, endless games and endless debates. To Tiago Almeida, Francisco Lopes, Diogo Silva, João Ribeiro, Nelson Carvalho, Sofia Santos, Ana Marques, Marta Almeida, Patrícia Oliveira, Ricardo Magalhães, Mónica Torres, Raquel Pinto and Sílvia Sousa, this is it, the end of a cycle.

Tiago Filipe Sousa Gonçalves 
"Any new technology, if it's used by evil people, bad things can happen. But that's more a question of the politics of the technology."

Geoffrey Hinton 


\section{Contents}

1 Introduction 1

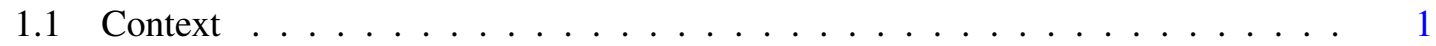

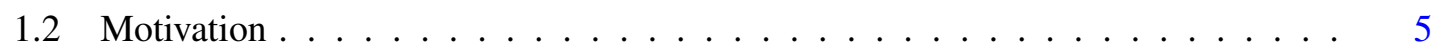

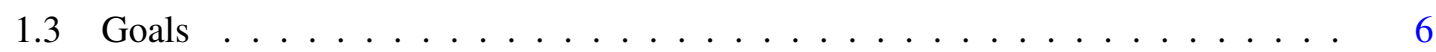

1.4 Dissertation Structure . . . . . . . . . . . . . . . . . . . 6

2 Literature Review 9

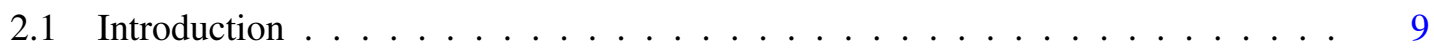

2.2 BCCT Assessment . . . . . . . . . . . . . . . . . . 11

2.3 Computer-Aided Aesthetic Classification of BCCT Outcomes . . . . . . . . . . 15

2.4 Towards a Deep Learning Approach to Keypoint Detection . . . . . . . . . . . . 17

2.5 Conclusions . . . . . . . . . . . . . . . . . . . . . 21

3 Image Segmentation for Breast Contour Detection 23

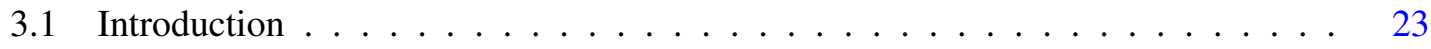

3.2 Traditional Approaches . . . . . . . . . . . . . . . . . . . . . . . 23

3.3 Deep Learning Approaches . . . . . . . . . . . . . . . . . . . . . . . . . . 24

3.4 Segmentation for Breast Contour Detection . . . . . . . . . . . . . . . . . . 29

3.5 Implementation and Results . . . . . . . . . . . . . . . . . . . . . . 29

3.6 Conclusions . . . . . . . . . . . . . . . . . . . . . 31

4 Quality-Driven Keypoint Detection 33

4.1 Introduction . . . . . . . . . . . . . . . . . . 33

4.2 State of the Art on Deep Quality-Driven Architectures . . . . . . . . . . . . 33

4.3 Quality-Driven Architectures for Keypoint Detection . . . . . . . . . . . . . . 37

4.4 Implementation and Results . . . . . . . . . . . . . . . . . . . . 40

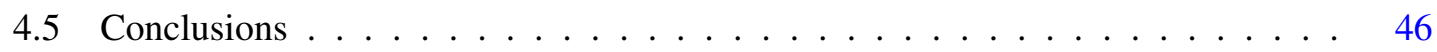

5 A Web Application for Automatic Keypoint Detection 49

5.1 Introduction . . . . . . . . . . . . . . . . . . . . . . . 49

5.2 Use-Cases and Proposed Architecture . . . . . . . . . . . . . . . . . 50

5.3 Implementation and Prototype . . . . . . . . . . . . . . . . 51

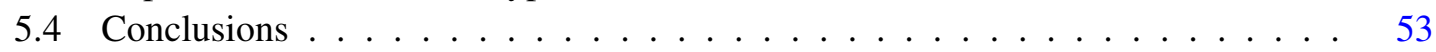

6 Conclusions and Future Work $\quad 55$

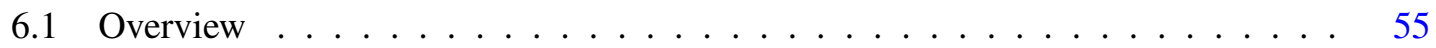

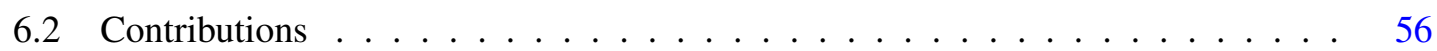

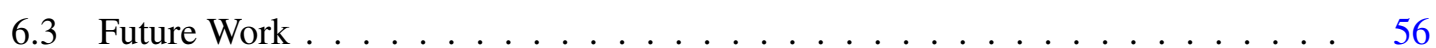




\section{List of Figures}

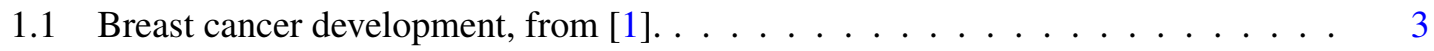

1.2 Differences between lumpectomy and mastectomy, respectively, from [2;3]. . . . 5

2.1 BAT $^{\circledR}$ interface, from $[4] . \ldots \ldots \ldots \ldots \ldots$

2.2 BCCT.core interface, from [5]. . . . . . . . . . . . . . . . . 15

2.3 Localization of the keypoints in a digital woman photograph (after BCCT). . . . 17

2.4 Proposed architecture for deep keypoint detection, from [6]. . . . . . . . . . . . 19

2.5 Example images from PORTO Dataset. . . . . . . . . . . . . . . . . 20

2.6 Example images from VIENNA Dataset. . . . . . . . . . . . . . . . . . . 20

2.7 Example images from TSIO Dataset. . . . . . . . . . . . . . . . . . . . . 20

3.1 The concept of FCN, from [7]. . . . . . . . . . . . . . . . . . . . . . . 24

3.2 U-Net architecture, from [8] . . . . . . . . . . . . . . . . . . . 26

$3.3 \mathrm{GCN}$ architecture, from [9]. . . . . . . . . . . . . . . . . . . . . . 27

3.4 DeepLabv3+ architecture, from [10]. . . . . . . . . . . . . . . . . . 28

4.1 Gossip Network architecture, from [11]. Note that 1-Mask is the complement of the Mask. . . . . . . . . . . . . . . . . . . . . . . . . . . 34

4.2 Stream module of the Gossip Network architecture, from [11]. . . . . . . . . . . 35

4.3 Gossip block of the Gossip Network architecture, from [11]. . . . . . . . . . . . 35

4.4 Iterative segmentation refinement network architecture (with skin lesion image as example), from [12]. . . . . . . . . . . . . . . . . . . . 36

4.5 U-Net [8] with quality prediction module, from [12]. . . . . . . . . . . . . 37

4.6 Keypoint's quality inference network architecture. . . . . . . . . . . . . . . 38

4.7 CNN Module's architecture. . . . . . . . . . . . . . . . . . . . . 38

4.8 MLP's architecture. . . . . . . . . . . . . . . . . . . . . . . . . . . . . 39

4.9 Proposed architecture for the iterative keypoint refinement network. . . . . . . . 40

4.10 Differences between the application of 1000 and 5000 backpropagation steps to the candidate keypoints which were the result of prediction of the model described in [6] in images of the test set. . . . . . . . . . . . . . . . . .

4.11 Differences between the application of 1000 and 5000 backpropagation steps to the candidate keypoints which were the result of random generated values. . . . .

4.12 Differences between the application of 1000 and 5000 backpropagation steps to the candidate keypoints which were coordinates with value $0 \ldots \ldots \ldots$

4.13 Evolution of candidate keypoint coordinates (predictions from [6]), from the initial to the adapted states, against the ground-truth. . . . . . . . . . . . . . .

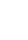
14 15 9 0 
4.15 Evolution of candidate keypoint coordinates (coordinates with value 0), from the initial to the adapted states, against the ground-truth . . . . . . . . . . . 46

5.1 Proposed Entity-Relationship model of the application's database. . . . . . . . . 50

5.2 Web application's main interface. . . . . . . . . . . . . . . . 51

5.3 Web application's images' functions interface. . . . . . . . . . . . . . . . 52

5.4 Web application's plot keypoints interface. . . . . . . . . . . . . . . 52

5.5 Proposed work-flow for the application. . . . . . . . . . . . . . 54 


\section{List of Tables}

2.1 Patient, tumour and treatment-related factors, from [13] . . . . . . . . . .

2.2 Average error distance for endpoints, breast contours and nipples measured in pixels, from [6]. Best results are highlighted in bold. . . . . . . . . . . . .

3.1 Examples of masks obtained with GCN and U-Net. Results are divided in good or bad, in comparison with the ground-truth masks. . . . . . . . . . . . . . . .

3.2 Average Dice Coefficient results on test set for U-Net and GCN models. Best result is highlighted in bold. . . . . . . . . . . . . 31

4.1 CNN Module layer's hyperparameters (VGG16 not included). . . . . . . . . . . 41

4.2 MLP layer's hyperparameters. . . . . . . . . . . . . . . . . . . . . 41

4.3 Results for the backpropagation applied to the candidate keypoints which were the result of the prediction obtained with the model described in [6] in images of the test set. Best results are highlighted in bold. . . . . . . . . . . . . . . .

4.4 Results for the backpropagation applied to the candidate keypoints which were the result of random generated values. Best results are highlighted in bold. . . . . . . .

4.5 Results for the backpropagation applied to the candidate keypoints which were coordinates with value 0 . Best results are highlighted in bold. . . . . . . . . . 42

4.6 Intermediate MLP layer's hyperparameters. . . . . . . . . . . . . . . . . . . . . . . . 44

4.7 Quality MLP layer's hyperparameters. . . . . . . . . . . . . . . . . . . . . . . . . 44

4.8 Keypoints MLP layer's hyperparameters. . . . . . . . . . . . . . . . . . . 44

4.9 Results for the iterative refinement model applied to the candidate keypoints which were the result of the prediction obtained with the model described in [6] in images of the test set. . . . . . . . . . . . . . . . .

4.10 Results for the iterative refinement model applied to the candidate keypoints which were the result of random generated values. . . . . . . . . . . . . .

4.11 Results for the iterative refinement model applied to the candidate keypoints which were coordinates with value $0 . \ldots \ldots \ldots \ldots \ldots$ 


\title{
Abbreviations and Symbols
}

\author{
ASPP Atrous Spatial Pyramid Pooling \\ BAD Breast Area Difference \\ BAT Breast Analysing Tool \\ BCD Breast Contour Difference \\ BCE Breast Compliance Evaluation \\ BCS Breast Cancer Conservative Surgery \\ BCCT Breast Cancer Conservative Treatment \\ BMI Body Mass Index \\ BOD Breast Overlap Difference \\ BRA Breast Retraction Assessment \\ BSE Breast self-exam \\ BSI Breast Symmetry Index \\ CAD Computer-Aided Diagnosis \\ CBE Clinical breast examination \\ CNN Convolutional Neural Network \\ DC Dice Coefficient \\ DCIS Ductal carcinoma in situ \\ DCL Dice Coefficient Loss \\ DNN Deep Neural Network \\ FCN Fully Convolutional Network \\ GCN Global Convolutional Network \\ GNN Graph Neural Network \\ IBC Inflammatory breast cancer \\ IDC Invasive (infiltrating) ductal carcinoma \\ IMF Infra-Mammary Fold \\ ILC Invasive (infiltrating) lobular carcinoma \\ IQT Inner Quadrant Transition \\ LBC Lower Breast Contour \\ LCIS Lobular carcinoma in situ \\ LIQ Lower Inner Quadrant \\ LMS Least Mean Squares \\ LOQ Lower Outer Quadrant \\ LQT Lower Quadrant Transition \\ MAE Mean Absolute Error \\ MLP Multilayer Perceptron \\ MSE Mean Squared Error \\ NAC Nipple-Areola Complex \\ OQT Outer Quadrant Transition
}


PSP Pyramid Scene Parsing

QOL Quality of Life

ROI Region of Interest

RNN Recurrent Neural Network

SQT Superior Quadrant Transition

SVM Support-Vector Machine

UIQ Upper Inner Quadrant

UNR Upward Nipple Retraction

UOQ Upper Outer Quadrant 


\section{Chapter 1}

\section{Introduction}

Contents

1.1 Context $\ldots \ldots \ldots \ldots \ldots \ldots \ldots \ldots \ldots \ldots \ldots \ldots$

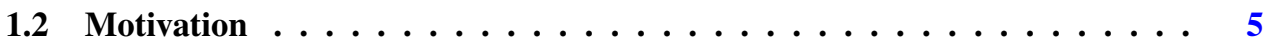

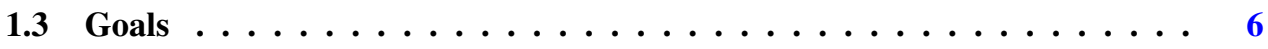

1.4 Dissertation Structure $\ldots \ldots \ldots \ldots \ldots \ldots \ldots$

\subsection{Context}

\subsubsection{An Overview of Worldwide Cancer}

Cancer, a group of diseases characterized by the uncontrolled growth and spread of abnormal cells [14], is a major public health problem worldwide [15], being the leading cause of death in economically developed countries and the second leading cause of death in developing countries [16;17], with approximately 14 million new cases and 8 million cancer-related deaths in 2012 [18]. Incidence is defined as the number of new cases that occur in a specific period and geographic area, conveyed either as an absolute number of cases per annum or as the rate per 100,000 persons per year [19]. It is also important to mention that rates are used to approximate the average risk of developing cancer in a given year and allow comparisons between countries and world regions [19]. The increase in the incidence of cancer in both economically developed and developing countries can be explained by the significant changes in reproductive behaviour, the use of exogenous hormones, differences in weight, exercise, diet, alcohol and tobacco consumption, as well as the result of population ageing and growth [16;20]. Gender is also an important factor to bear in mind when dealing with cancer. Among men, the five most common (sites of) diagnosed cancers were the lung, prostate, colorectum, stomach and liver, while, among women, the five most common were the breast, colorectum, lung, cervix and stomach. On the other hand, incidence also sees significant changes when the analysis is made taking into account gender. Among men, lung and prostate cancer have the highest values, while, among women, it is breast cancer 
that leads this ranking, being immediately followed up by colorectal cancer [18]. It will be on breast cancer that this work will be centred.

\subsubsection{Breast Cancer}

Cancer occurs when body cells begin to grow wildly, dividing in an aberrant fashion crowding normal cells. These cells, which are designated by cancer cells, also have the ability to break off the primary tumour (see Figure 1.1), enabling, thus, a spread of the disease through direct organ invasion, the lymphatic and/or the circulatory systems, in a process that is named metastasis (which can occur at the time of diagnosis or after patient has been disease-free after receiving treatment) [21]. Breast cancer is a malignant (or cancerous) tumour which starts from the cells of the breast tissue. As stated earlier, this type of cancer primarily affects women, but men may also be diagnosed, although it is uncommon [22]. On the other hand, it is possible that some women could develop breast lumps which are benign or non-cancerous abnormal growths that are not lifethreatening if they do not spread outside of the breast. These situations, however, augment the risk for a given person to develop breast cancer [23].

\subsubsection{Breast Cancer Types}

There are several types of breast cancer [23; 24]:

- Ductal carcinoma in situ (DCIS): the most common type of non-invasive breast cancer confined to the ducts. Generally, every woman with cancer at this stage can be cured.

- Lobular carcinoma in situ (LCIS): starts in the milk-producing glands but does not go through the wall of lobules. Despite not being considered true cancer, having LCIS contributes to a woman's risk of developing cancer at a certain point in time.

- Invasive (infiltrating) ductal carcinoma (IDC): the most common type of breast cancer. Begins in a milk passage or duct, breaks through the wall of the duct and invades the tissue of the breast, from where it can spread to other body parts.

- Invasive (infiltrating) lobular carcinoma (ILC): which starts in the milk-producing glands or lobules, from where it can spread to other body parts.

- Inflammatory breast cancer (IBC): an infrequent and aggressive type of breast cancer, recognized for presenting a reddened swollen breast. Pathologically complex, this type of cancer not only has a ductal or lobular histology but also has cancer cells evident in the lymphatics of the skin, causing the inflamed appearance. The prognosis is, usually, very poor. 


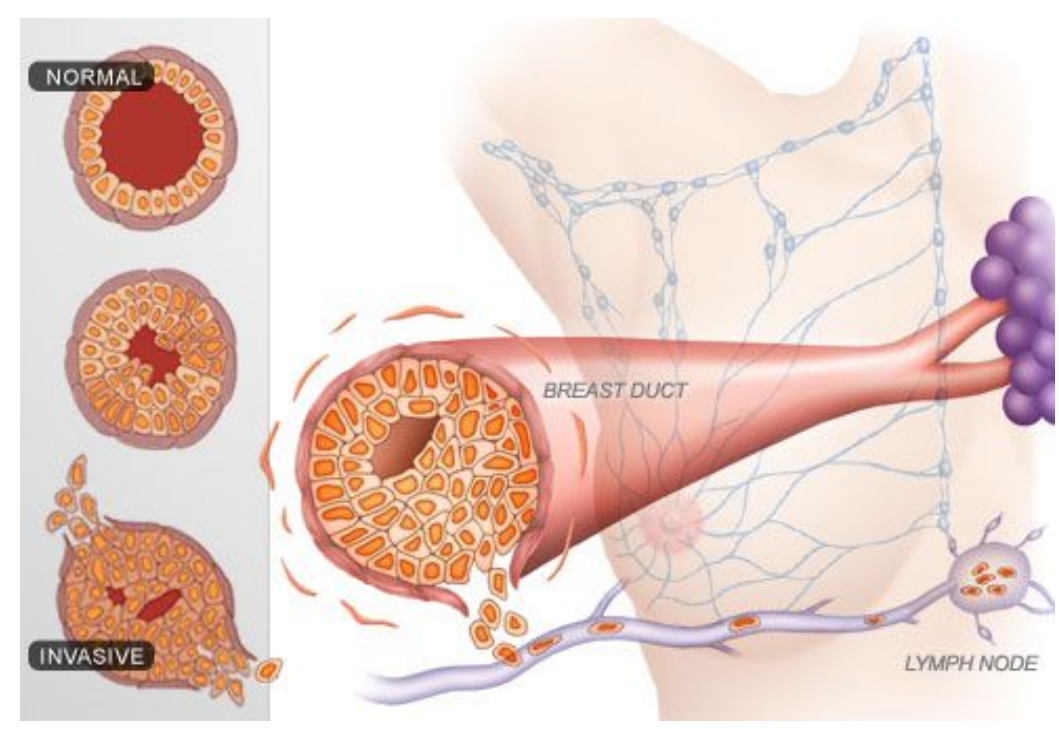

Figure 1.1: Breast cancer development, from [1].

\subsubsection{Breast Cancer Diagnosis}

The principal mission of cancer diagnosis and treatment is, indeed, detecting the malignancy prior to clinical appearance [23]. Actually, breast cancer is usually detected during a screen examination (before symptomatic manifestations) or after a woman notices a lump [25]. There are several diagnostic and screening measures specific to breast cancer that could vary, according to the person's age. These measures are normally published as official guidelines, that are updated from time to time $[25 ; 26]$. According to literature, these are the most relevant screening and/or diagnosis methods for breast cancer:

- Breast self-awareness: which is based on the assumption that every woman should be familiar with both the appearance and feel of their breasts and report any changes to their physician. Some studies show that this method has been at least as effective for detecting breast cancer as a structured breast self-exam (BSE) [27; 28; 29].

- Clinical breast examination (CBE): that consists on breast palpation by a clinician [30]. Although some authors state that this technique, along with mammography, could benefit the diagnosis of breast cancer [31;32], others state that this technique could also increase the number of false positives [33;25].

- Mammography: which is a low dose x-ray procedure that allows visualization of the internal structure of the breast. This method is usually essential for early breast cancer detection. This method is usually for women under the age of 50 and/or those with dense breast tissue $[34 ; 35 ; 36]$.

- Breast ultrasound: a method that utilizes sound waves to determine if an area of concern on a mammogram is cystic or solid. Although not being a specific method to perform 
breast cancer screening, it is useful to evaluate abnormal findings from a mammogram or physical exam. Usually, almost all cystic masses are benign while solid masses may need more evaluation. This technique has shown some promising results, although it has been demonstrated that also increases the likelihood of false-positive results $[25 ; 23 ; 37 ; 38]$.

- Breast Magnetic Resonance Imaging (MRI): that uses magnetic fields to produce detailed cross-sectional images of tissue structures [25; 23]. A contrast agent (usually, is gadolinium) is injected into a vein in order to provide better soft tissue contrast, thus, improving the ability to capture detailed images of the breast tissue [39]. Along with mammography, MRI could help the development of both screening and treatment of breast cancer.

\subsubsection{Breast Cancer Treatments}

In general, breast cancer treatment decisions are made together by the patient and physician regarding some important variables such as stage and biological characteristics of cancer, patient's age, menopausal status, preferences, and the risks and benefits of each possible option. It is also important to acknowledge that all cancer types have different behaviours which lead to the need for a multimodal approach on treatment, such as surgery, radiation and/or chemotherapy. Breast cancer is considered a systemic disease from diagnosis, so, it is extremely important to determine cancer staging (how widespread cancer is at the time of diagnosis), a factor which will influence the treatment decision from that moment $[23 ; 25]$. Surgery is usually the primary approach in terms of treatment. Its main objectives are to remove cancer and to determine the stage. It is possible to specify surgery in the following types (see Figure 1.2):

- Partial (segmental) mastectomy or lumpectomy: which is based on the removal of the cancerous tissue with a rim of healthy tissue (free margin). This approach is also known as Breast Cancer Conservative Surgery (BCS) or Breast Cancer Conservative Treatment (BCCT) [40; 23; 25].

- Simple or total mastectomy: which includes the removal of the entire breast, but not of the lymph nodes under the arm or muscle tissue from beneath the breast [23; $25 ; 41]$.

- Modified radical mastectomy: based on the removal of the entire breast without the chest muscle and removal of the first two stages of lymph nodes under the arm [23;25].

- Radical mastectomy: which consists of the extensive removal of the entire breast, lymph nodes, and chest wall muscles under the breast [23; 25; 42].

After surgery, patients usually undergo radiation or systemic therapies in order to increase the effectiveness of the treatments. Radiation therapy consists in the use of high-energy rays, beams or particles to destroy cancer cells, and is commonly used after surgery to remove cells that may remain in the breast, chest wall, or underarm area [21]. It is important to take into account that the duration of the therapy and the type of radiation that will be used will always depend on the type of performed surgery [43]. System therapy is a type of treatment that uses the bloodstream 

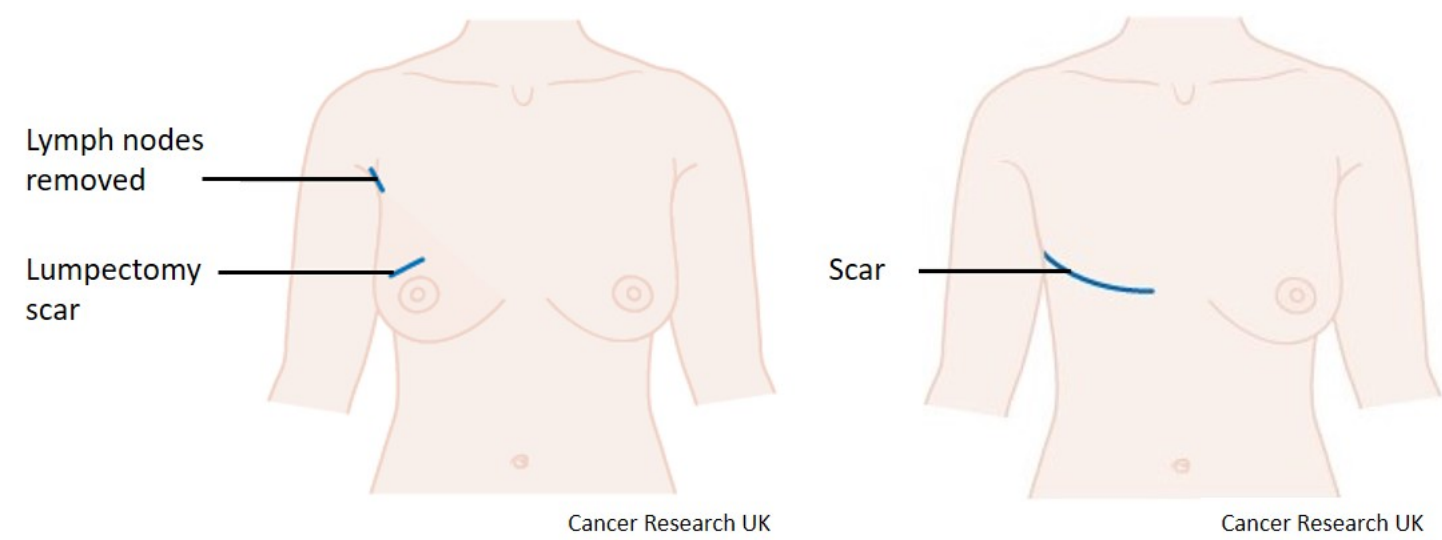

Figure 1.2: Differences between lumpectomy and mastectomy, respectively, from [2;3].

to reach body sites, which means that it will treat some parts and affect others. This is actually a group of different approaches, including chemotherapy, hormone therapy and targeted therapy, which have different mechanisms [25]. Chemotherapy is a treatment that uses medications to stop the growth of cancer cells (these drugs address cells that divide quickly and cancer cells fall within that group), either by killing them or preventing them from dividing [21]; this type of treatment is usually applied to reach known or unknown metastases that may have spread to other distant organs [23]. Hormone therapy, or anti-estrogen therapy, is a method that will lower the production of this hormone or block its effect, regarding the fact that estrogen is also known for promoting the growth of breast cancer cells in women [44]. Targeted therapies are the most recent approach related with breast cancer combat; this type of focused treatment has been evolving with the research on cancer genetics and with the discovery of the correlation with some genes and breast cancer. This type of therapy usually addresses specific proteins, recognized for promoting cell growth and for being in abnormal concentrations in cancer cells [45]. Depending on the patient's situation, these treatments can also be employed before surgery. After treatment, followup visits should take place in order to have constant monitoring of the disease status.

\subsection{Motivation}

Despite being a highly mutable and rapidly evolving disease, it is estimated that most breast cancers (approximately $90 \%$ of the cases) are treatable and curable if detected on early staging and given the best treatment [20]. Nowadays, with implemented breast cancer routine screening habits (that promote early diagnosis with regular medical prevention consultations concerning the presence or absence of risk factors) [46; 47], it is possible to do proper management of the disease, in case it appears, and to develop a better clinical plan to treat it. This means that most breast cancers can now be treated using conservative approaches, i.e., BCCT, contributing, thus, for local control of the disease, with similar survival rates to those obtained with mastectomy, although, with better cosmetic results $[48 ; 41 ; 20]$. This leads to a focused concern on aesthetic results and the patient's 
Quality of Life (QOL) which have become fundamental objectives within this type of treatment. Nonetheless, results depend on a variety of factors (e.g., patient, tumour, type of surgery, factors related to treatment, type of therapies) that will influence the outcomes [49; 50]. This assessment of the cosmetic result has indeed become essential to the institutions that provide breast cancer treatment since it is a mean of evaluation of one of the aspects of treatment quality while helping with the refinement and the improvement of current techniques. Moreover, the definition of an objective evaluation standard for the cosmetic outcome of BCCT (in analogy with the oncological outcome) has become crucial, due to the fact that with the development of new oncoplastic techniques it becomes necessary to have an objective method to compare cosmetic results $[51 ; 20]$. Current objective methods, BAT ${ }^{\complement}$ [52] and BCCT.core [5], perform the aesthetic assessment of BCCT based on asymmetry measurements; however, they require information that must be given by the user and none of them has been considered the gold standard for this task. Therefore, there is a need for an automatic method that is able to perform this assessment by simply receiving data (i.e., images) as input. To accomplish this, one must develop an algorithm that is able to automatically detect breast fiducial points (i.e., keypoints), which are needed to compute asymmetry measurements and to perform the assessment. Moreover, with the current developments on machine learning and deep learning techniques for biomedical image classification and segmentation [53], it is believed that this should be the path to follow, regarding the investigation on this topic. In fact, results obtained by Silva et al. [6] show that there is more work to do in terms of investigating both automatic fiducial points detection and the best deep learning model to do such task. This thesis project will focus precisely on that investigation.

\subsection{Goals}

The main goals of this project are:

1. The investigation of different deep learning approaches for fiducial point detection.

2. The development of a web application with the best-researched technique as an online service, based on HTML5, CSS, JavaScript and PHP.

\subsection{Dissertation Structure}

Besides Introduction, this thesis contains five more Chapters.

Chapter 2 reviews the current methodologies for aesthetic assessment of BCCT and the application of deep learning architectures to this task.

Chapter 3 focuses on a comparative study with different deep learning architectures for image segmentation, exploring their potentialities for being a means to obtain better results on breast contour detection.

Chapter 4 discusses a novel methodology towards the use of deep learning architectures for breast contour keypoint detection. 
Chapter 5 presents a prototype of a web application capable of integrating keypoint detection algorithms as a web service for healthcare professionals.

Chapter 6 shows the main conclusions obtained with the work developed under the scope of this thesis and gives suggestions on posterior future work. 


\section{Chapter 2}

\section{Literature Review}

\section{Contents}

2.1 Introduction $\ldots \ldots \ldots \ldots \ldots \ldots \ldots \ldots \ldots \ldots$

2.2 BCCT Assessment $\ldots \ldots \ldots \ldots \ldots \ldots \ldots \ldots \ldots \ldots \ldots$

2.3 Computer-Aided Aesthetic Classification of BCCT Outcomes . . . . . . 15

2.4 Towards a Deep Learning Approach to Keypoint Detection . . . . . . . . 17

2.5 Conclusions . . . . . . . . . . . . . . . . . 21

\subsection{Introduction}

\subsubsection{The Importance of BCCT}

As stated in Chapter 1, breast cancer is the leading cause of cancer death in women worldwide, while being the most frequently diagnosed type of cancer. However, if detected in an early stage and properly treated, a vast majority (estimated $90 \%$ of the cases) of breast cancers are curable, leaving, thus, space for the study of the impact that aesthetic appearance of the breast has on women [20]. Actually, this is partly due to the execution of good practices, such as routine screening, which promotes early diagnosis with regular medical prevention consultations, always concerning the presence or absence of risk factors. The increasing use of the BCCT approach over the last few years [5] has made local control of the disease possible, with similar survival rates to those obtained with mastectomy, but with better cosmetic results [48; 41]. Also, in this type of treatment, objectives such as patient's QOL and the aesthetic results are fundamental, even though, results are dependent on a variety of factors which will contribute to different outcomes [50;49]. Considerable research on BCCT techniques has been done, but diverse aesthetic results are still common, so, the assessment of the cosmetic result has become crucial for institutions that perform breast cancer treatment, as mean of evaluation of the treatment quality and of improvement of working practices $[50 ; 5 ; 20]$. In addition, the development of novel oncoplastic techniques in breast conservation requires the existence of an objective method to assess the cosmetic results. 
Table 2.1: Patient, tumour and treatment-related factors, from [13].

\begin{tabular}{ll}
\hline Patient dependent factors & Tumor and treatment factors \\
\hline Age & Incision size $(\mathrm{cm})$ \\
Height $(\mathrm{cm})$ & Tumor size (mm) \\
Weight $(\mathrm{kg})$ & Incision type (radial, sulcus, periareolar, curved) \\
Thorax perimeter (cm) & Specimen weight (g) \\
Body Mass Index (BMI) & Side (left, right) \\
& Tumor location: \\
& Upper Outer Quadrant (UOQ) \\
& Upper Inner Quadrant (UIQ) \\
& Lower Outer Quadrant (LOQ) \\
& Lower Inner Quadrant (LIQ) \\
Breast size (radius cm) & Outer Quadrant Transition (OQT) \\
& Superior Quadrant Transition (SQT) \\
& Inner Quadrant Transition (IQT) \\
& Lower Quadrant Transition (LQT) \\
& Type of intervention (tumorectomy or tumorectomy \\
Bra cup size (A, B, C, D) & with sentinel node biopsy or with axillary dissection) \\
Age of menarche & Scar visibility (not, slightly, very visible) \\
Number of pregnancies & Surgeon (general, breast $\rightarrow$ 150 cases/year) \\
Age of first pregnancy & Chemotherapy (yes, no) \\
Oral contraceptives (yes, no) & Hormone therapy (yes, no) \\
Menopause (yes, no) & Follow-up (months)
\end{tabular}

Actually, while the oncological outcome of BCCT procedures can be objectively assessed, the cosmetic outcome does not yet have an evaluation standard [51; 54].

\subsubsection{Important Factors to the Cosmetic Outcome}

It is important to bear in mind that, although BCCT has an identical meaning worldwide, it is not a standardized procedure [49], due to the fact that there are some technical variations related both to surgery and radiotherapy. In order to clarify what is considered a good aesthetic, one must have a notion of the factors that may influence the results [54]. Generally, these are divided into patient, tumour and treatment-related factors, as can be seen in Table 2.1. With the development of new oncoplastic techniques, new factors, especially related to the surgery will arise, so, it will be imperative to have a clear idea of which factors influence the cosmetic result in order to achieve a reproducible way of measuring these outcomes, independently of the used technique. Then, it will be possible to establish a correlation between results with practice and, subsequently, improve results $[55 ; 20]$.

\subsubsection{Cosmetic Assessment Parameters}

Generally, the aesthetic result of BCCT is done by an observer who identifies and evaluates colour, shape, geometry, irregularity and roughness of the treated breast in comparison with the untreated 
breast. This relies on the assumption that better results correspond to more similar breasts; this is considered to be a much more practical approach and a method that fits the evolution and the appearance of new emerging oncoplastic techniques, where, usually, both breasts are submitted to surgery, leading, consequently, to a more demanding comparison [20; 54]. Regarding factors that contribute to the global aesthetic result, asymmetry in size is, perhaps, the most important, that impacts the global cosmetic result and primarily depends on the amount of excised tissues, when surgery is unilateral $[56 ; 57 ; 58 ; 59 ; 20]$. Surgery and radiotherapy derived fibrosis, with upward retraction of the inferior mammary sulcus and the nipple-areola complex (NAC) can also have a consequence in the evaluated symmetry without impairing the size of the breast. Other factors, such as scar visibility and length, hyperpigmentation of the treated breast or the hypopigmentation of the NAC, are also taken into account $[56 ; 58 ; 59 ; 57 ; 60 ; 20]$.

\subsection{BCCT Assessment}

\subsubsection{Patient Evaluation}

To accomplish this task, the direct observation of patients is promoted by the vast majority of the professional community involved in BCCT as the most complete form of cosmetic evaluation, since physicians can have a better global appreciation of the results while checking other factors such as skin atrophy and oedema of breast and arm [50; 61]. However, photographs are more practical because it is possible to save them permanently, visualize them when necessary and sharing them, allowing observations by different physicians [62; 63]. With the advent of technology evolution, the use of digital photography has made the process even easier and less expensive, while maintaining picture quality $[63 ; 52]$.

\subsubsection{Assessment Methodologies}

\subsubsection{Scales}

Historically, it was Jay Harris, in 1979, the one who had introduced a subjective overall cosmetic score, that would become a standard in conservation breast procedures: the Harvard scale [64]. This scale classifies the overall cosmetic result in four classes:

1. Excellent: if the treated breast is nearly identical to the untreated one.

2. Good: if the treated breast has some differences when compared with the untreated one.

3. Fair: if the treated breast is clearly different from the untreated one, but not seriously distorted.

4. Poor: if the treated breast is seriously distorted.

Several other authors have proposed different methods, to obtain a more complete and discriminative classification, including, for instance, other factors such as skin oedema and thickening, 
mammary fibrosis, retraction and telangiectasias [65; 66; 67]. Each factor would be assigned an individual score, and, then, the sum of scores would result in a final score that would allocate the patient into one of the four classes described above. Also, more sophisticated scores to measure the aesthetic result of BCCT have been described, although, none of them was considered to be the best alternative to the Harvard scale $[66 ; 68 ; 20]$.

\subsubsection{Subjective Methods}

One can divide assessment methods into subjective and objective methods. Subjective methods usually include patients' self-evaluation and evaluation by a single observer or by a panel of observers. Regarding patients' self-evaluation, one can state that this is the easiest way to analyse the cosmetic outcome of BCCT (and the one that best translates the psychosocial adaptation of patients to the result [59]), however with low reproducibility, since factors such as age, socio-economic status or the fear to express criticism towards treatment or responsible caregiver will have a direct impact on how women see themselves after the treatment, having direct consequences on the final evaluation. On the other hand, it is always important to have feedback from patients on aesthetic results $[69 ; 50]$, since they are the principal stakeholder on the treatment process. Generally, the subjective assessment is made by one or more observers, who focus directly on patients or on photographic representations of them [70; 71]. The final aesthetic result is then assessed, using the Harvard scale, where results are ranked according to comparisons between the treated breast and the untreated one. Even though there are some problems regarding the interpretation of results from studies which use this type of assessment (e.g., impartiality from professionals involved in the treatment is hard to guarantee), observer evaluation is the most frequently used method. Also, issues regarding the impracticality and the invasion of patients' privacy, deeply connected with this type of evaluation, are not negligible; for instance, if the evaluation is carried out by one or more observers involved in the treatment process and at different monitoring stages, the discomfort caused to patients may be high, even if using digital images and e-mail evaluation, since the process could take months or even years, when performed in a large number of patients with multiple observers [72].

\subsubsection{Objective Methods}

In an attempt to overcome this lack of objectivity and reproducibility, objective assessment methods were introduced. In fact, the introduction of objective methods was done by Richard Pezner, in 1985, with the first objective measure to evaluate asymmetry, one of the aspects of cosmesis: Breast Retraction Assessment (BRA) [58]. The reinforcement of the importance of objective measures was also a significant contribution from this author, with the demonstration that observer consensus of cosmetic outcome is difficult to obtain [61]. This line of thought was followed by other authors, which contributed with new measurements: Van Limbergen et al. proposed two new asymmetry measurements, the Lower Breast Contour (LBC) and the Upward Nipple Retraction (UNR) [56]; Tsouskas and Fentiman described the Breast Compliance Evaluation (BCE), which 
is the difference between the distance from the nipple to the Infra-Mammary Fold (IMF) [73]; Noguchi et al. introduced innovative approaches on objective and subjective evaluations, with the utilisation of Moiré topographic to analyse the differences between the displayed curves in both breasts, as the objective part, the determination of other parameters (such as skin changes and scar) with the evaluation of observers, as the subjective part, and the sum of parts as the final result [74]. This last approach was well accepted in the community and was adopted by other authors as well. The simple sum of individual indices, objective and subjective, failed to acknowledge that different aspects have different contributions to the overall result; the reliance on the subjective assessment of individual aspects made the final decision irreproducible; the manual measurement made them partially subjective, and, because of that, the process was still time-consuming and hard to implement under routine clinic conditions [20]. To overcome such conditions, new generations of methods have been developed: two-dimensional methods and three-dimensional methods. Since this thesis is under the scope of two-dimensional methods, this Chapter will be focused on them. These software-based methods came up with the main idea of predicting the global aesthetic result since they are based on different individual characteristics which are automatically and objectively extracted from patient photographs. These approaches explore the ability that computational methods have to provide an effective, easy, fast, reliable and reproducible tool to evaluate the consequences of breast cancer patient care [20]. For these methods to work, a database of patient images evaluated subjectively by a panel of experts, with classifications that serve as ground-truth is required (and is also a way of developing new methods and compare results). Regarding twodimensional novel methods, one must talk about Breast Analysing Tool - BAT ${ }^{\complement}$ from Fitzal et al. [52] and BCCT.core from Cardoso and Cardoso [5]. Concerning BAT ${ }^{\complement}$, Fitzal et al. proposed the Breast Symmetry Index (BSI) to evaluate the cosmetic outcome of BCCT (see Figure 2.1). With $\mathrm{BAT}^{\complement}{ }^{\complement}$, they were able to measure differences between left and right breast sizes from a patient's digital picture. The sum of all differences will output the BSI score, which has the capability of measuring size differences in between breasts. BSI was reproducible and could be used by both experts and non-experts with the same results, while highly correlating with subjective votes from experts and significantly differentiating between good and fair cosmetic outcomes [52]. On the other hand, BSI could not further differentiate between excellent and good or fair and poor cosmetic outcomes, while there was not a correlation between the BSI index and the patients' selfevaluation [20]; even though, Krois et al. were able to improve the correlation between subjective and objective BSI values, establishing, thus, new standards on breast symmetry evaluation [4]. Concerning BCCT.core (see Figure 2.2), it is important to state that it is a computer-aided medical system that performs automatic feature extraction from patient photographs, in order to capture some of the relevant factors for the overall cosmetic result. To do a proper classification, it is necessary to obtain a concise representation of a BCCT image, based on asymmetry, colour differences and scar visibility features. To describe breast asymmetry, several features were taken into consideration [5]:

- Breast Retraction Assessment (BRA): which quantifies the difference in nipple position between both breasts and reflects the degree of breast retraction. 


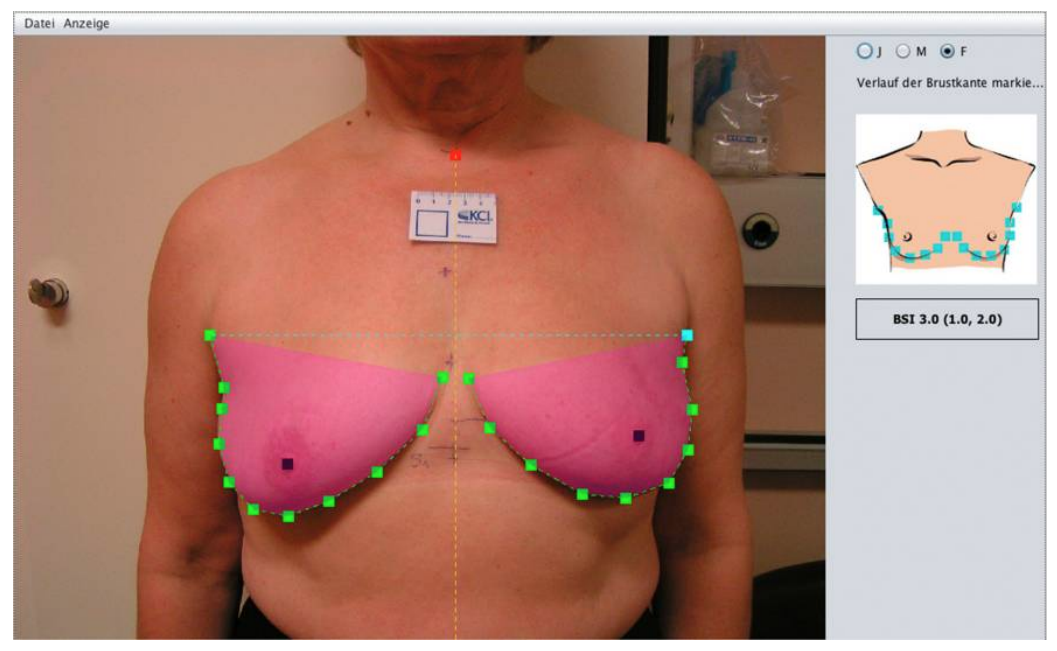

Figure 2.1: BAT $^{\complement}$ interface, from [4].

- Lower Breast Contour (LBC): which is the difference between levels of inferior breast contour.

- Upward Nipple Retraction (UNR): which is the difference between nipple levels.

- Breast Compliance Evaluation (BCE): that is the difference between left and right nipple to IMF distance.

- Breast Contour Difference (BCD): that is the difference between lengths of left and right breast contours.

- Breast Area Difference (BAD): that is the difference between areas of the left and right breasts.

- Breast Overlap Difference (BOD): that is the non-overlapping of the two breasts after flipping one of them along a vertical line and making coincident both points of junction with the thorax.

Dimensionless features derived from the previous ones were also proposed [5]. To extract their asymmetry features, it is necessary to mark some specific keypoints in the image, using BCCT.core software: sternal notch, the scale mark, the nipples and left and right breasts contours, adjusted with an active contour based on splines with control points. Also, each keypoint can be manually annotated and adjusted by the user. 


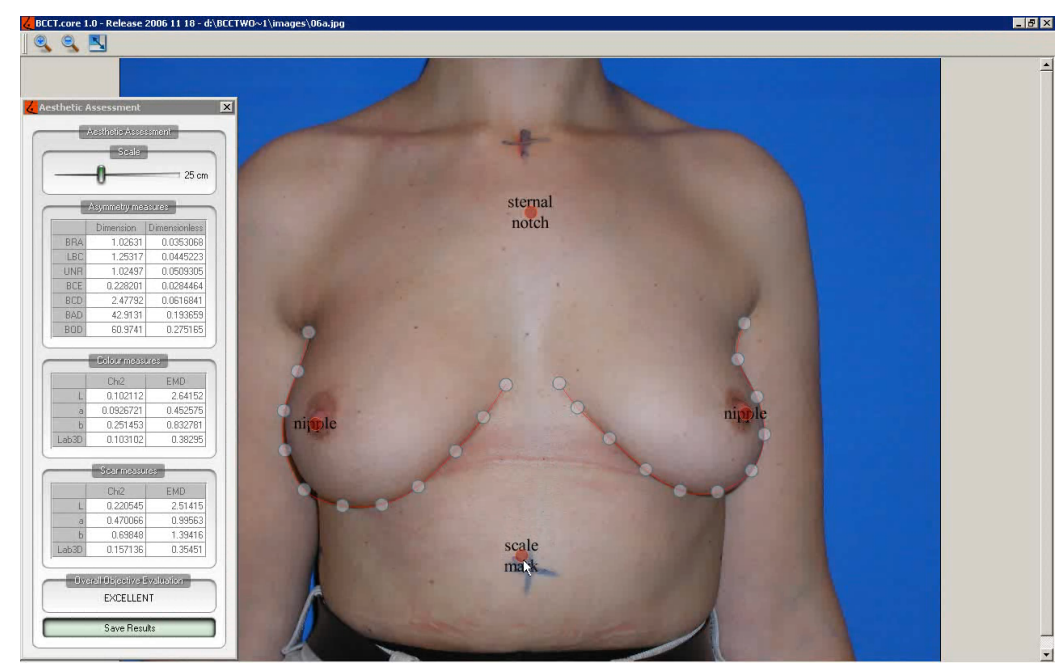

Figure 2.2: BCCT.core interface, from [5].

\subsection{Computer-Aided Aesthetic Classification of BCCT Outcomes}

\subsubsection{Keypoint Detection}

\subsubsection{Breast Contour}

Cardoso and Cardoso [75] were pioneers on the automatic detection of fiducial points in photographs of women after BCCT. These, also known as keypoints, are important points in the photographs that serve as a reference for the computation of the essential features that determine the aesthetic assessment of BCCT. Until their work publication, this task only tackled detection on digital mammograms, on a side view of the breast [76]. In that publication, they discuss three breast contour detection methods: polynomial modelling, active contours and graph-based computation of shortest path. Polynomial modelling and active contours presented unsatisfactory results. In case of polynomial modelling, because of biased regression by the high number of contour pixels not belonging to the breast and polynomial's lack of flexibility to adjust to breast contour; in case of active contours, the computation time was excessive [75]. Based on this preliminary research, a graph-based method was presented. Assuming that the two endpoints of the breast contour are known (given by the user), the problem resides on finding the path between both endpoints that goes through the breast contour. To accomplish this, they first compute the gradient of the image, as a method of emphasizing the pixels of interest, and then, they model the gradient image as a weighted graph, by matching a node to each pixel and connecting two nodes with an arc on the graph, if the corresponding pixels are neighbours (8-connected neighbourhoods) on the image; the weight of each arc is a function of pixels values and pixels relative positions. To find the breast contour, the shortest path on the graph is then found. Important to note that the main assumption in this work is that the inner region of the breast is completely free of edges, so, by favouring the paths through edge pixels, it is reasonable to assume that the shortest path is, indeed, the path that returns the breast contour. Sousa et al. proposed an extension of this work by introducing 
prior knowledge (i.e., shape priors) to the process. The main idea was that this prior knowledge was useful to simplify the process of localizing the breast contour; several types of priors were studied: parametric (parabola, ellipse) and non-parametric (mask, unimodal). It was concluded that models with prior knowledge perform better than the base model and non-parametric models obtain better results than parametric models [77]. Years later, Lee et al proposed a model based on a parametric active contour, which also ends up enforcing a mathematical shape constraint based on the catenary curve [78]. A catenary is a perfectly flexible and inextensible string of uniform density supported by two distinct points [79; 80]; these curves have been used to model objects shaped like hanging strings, such as arches and suspension bridges [81]. The results obtained with this approach were comparable with the ones obtained by Sousa et al.. Still, all these methods require the manual annotation of the breast contour endpoints.

\subsubsection{Endpoints}

Following the work presented by Cardoso and Cardoso [75], Cardoso et al. proposed a method for the automatic detection of the endpoints [82]. Assuming that the photo contains only the torso of the patient, the position of the external endpoint of the breast contour can be assumed to be at the point of the body where the arm contour intersects the trunk contour. In photographs, however, patient's are in the arms-down position, the arm's contour is almost indistinguishable from the trunk's contour, so, in this case, the external endpoint of the breast contour is defined as the highest point of the trunk contour. The images are divided into two regions (bottom half and top half), which are subjected to distinct approaches, after gradient computation. For the bottom half, the computation of the shortest path between each point in the bottom and middle rows, both in direct and reverse directions, is performed; then, to get the position of the two trunk contours, all the paths that were not common in both directions and the ones with a cost superior to half of the maximum possible cost were discarded. For the top half of the image, a selection of the shortest path that does not result in a long sequence of consecutive pixels with low gradient is done, in order to continue the trunk contour from the middle of the image to the endpoints. After these computations, the endpoints are found; the internal endpoint is considered to be the middle point between the two external endpoints [82].

\subsubsection{Nipples}

Another keypoint of utmost importance for the aesthetic assessment is the nipple, concerning its importance on the asymmetry measure corresponding to breast retraction. With this in mind, in 2007, Udpa et al., proposed an automatic method to detect the nipples, which consists on the use of normalized cross-correlation with a template bank of variants of Gaussian and Laplace of Gaussian filters and probability map of likely nipple locations to reduce the number of false positives. After these steps, the nipples are selected as the regions of the left and right half of the images where the pixels have the highest intensity [83]. In 2015, Cardoso et al. came up with an alternative approach to the automatic detection of nipples, supported by two assumptions: at least 
a nipple candidate lies within the true areola (closed region enclosing the nipple) contour and the areola contour represents a closed path over pixels of a strong gradient. Actually, breast surface is generally characterized as featureless shape, so, the nipple should be the most prominent feature on it. On the other hand, many other characteristics can be confused by the nipple when using traditional feature detectors and, concerning the fact that the nipple belongs to the areola complex, this regional information can support nipple detection. The method starts by over-detecting nipple candidates. To achieve this, a corner descriptor is used to detect possible nipple locations and a closed contour method is applied to find areola contours around those points. The next step lies on finding one or more closed contours enclosing each candidate; to accomplish this task, four highlevel features (Harris corner quality factor; the average magnitude of the directional derivative of the contour; shape factor of the contour; equivalent diameter of the contour) are extracted. The best pair candidate/closed contour is selected by fusing the information from the contour and the nipple candidate, i.e., based on the extracted features a support-vector machine (SVM) classifier [84] was trained, achieving comparable results [85]. Figure 2.3 shows the localization of the keypoints in a digital woman photograph (after BCCT).

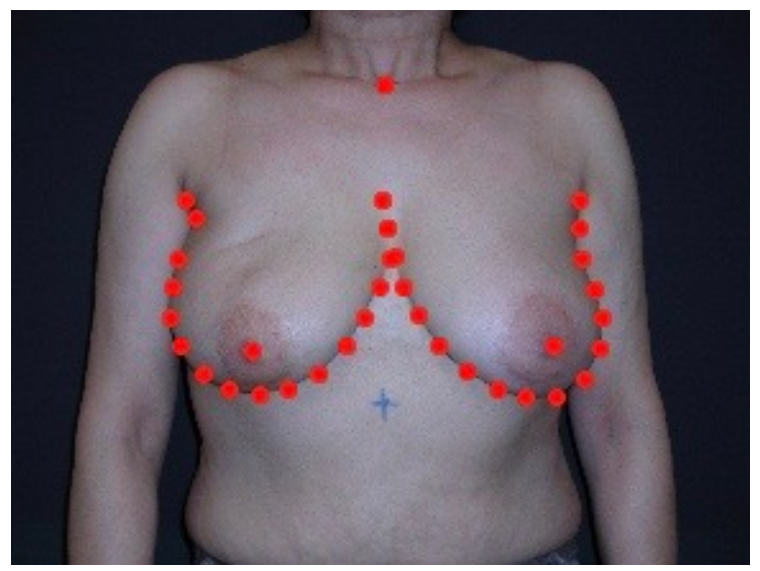

Figure 2.3: Localization of the keypoints in a digital woman photograph (after BCCT).

\subsection{Towards a Deep Learning Approach to Keypoint Detection}

When dealing with the traditional computer vision methods, previously explained, some keypoints (e.g., breast contour endpoints) are incorrectly detected, when the images have insufficient quality. Therefore, methods are not invariant to illumination and/or background variability. Also, there was no method proposed in the literature to detect the supra-sternal notch which is considered important for this problem. Moreover, due to the fact that the detection of different keypoints is being done with separate methods, one may be missing information on the context. Considering these drawbacks, Silva et al. have proposed the use of a deep neural network (DNN) for the keypoints detection task, taking into account that, with deep learning, it is possible to follow an integrated learning approach that uses the context information [6]. This thesis project will be focusing on this specific approach. 


\subsubsection{Proposed Architecture}

Generally, in the biomedical investigation, datasets are small, which might be considered a problem, regarding the fact that the deep learning models easily overfit on the existent data in these conditions, thus, not generalizing well for new breast images. There are available some regularization methods, operations or data augmentation techniques that could prevent this situation; however, there may be more interesting approaches that could be more specific for this regression problem. Actually, regarding keypoint detection with deep learning, there are two relevant works that presented interesting ideas: Cao al. [86], which brought the idea of learning part confidence maps and part confidence fields as a mean to detect keypoints in the end; Belagiannis et al. [87] which also proposed an architecture that first learns how to regress heatmaps (i.e., each keypoint is modelled with a Gaussian distribution) and, after iterative tuning on the training of heatmap regression, it is able to predict keypoint localizations. Taking these ideas into account, Silva et al. have proposed the generation of an intermediate representation (i.e., heatmaps), which consists on a fuzzy localization for the keypoints that are intended to be detected. The heatmaps are obtained using the segmentation model, U-Net [8]. For the keypoint regression, the strategy starts with the multiplication of the image with the refined output of the previous module, as a way of improving the fuzzy localization of keypoints, which will then enhance the exact keypoint detection. Therefore, the regression module predicts the keypoints' coordinates. This module is composed of three blocks:

1. VGG16 [88]: without the fully-connected layers, pre-trained with ImageNet and then finetuned with the available dataset.

2. Four convolutional layers: added to increase image processing and to decrease the size of feature maps before the dense layers.

3. Three dense layers: used to do the regression of the keypoint coordinates, corresponding to the keypoints that are part of the breast contour, the endpoints, nipples and supra-sternal notch.

The entire architecture is presented in Figure 2.4.

\subsubsection{Training Requirements}

This supervised learning process requires the ground-truth for the keypoints and the ground-truth for the heatmaps, which are created considering a Gaussian centered at each keypoint, with a pre-defined standard deviation. Also, with this approach, there are two different terms in the loss function: heatmap regression (a regularization term) and keypoint regression (the main goal). Therefore, the loss function is a linear combination of these two terms (Equation 2.1).

$$
\mathscr{L}=\mathscr{L}_{\text {heatmaps }}+\mathscr{L}_{\text {keypoints }}
$$




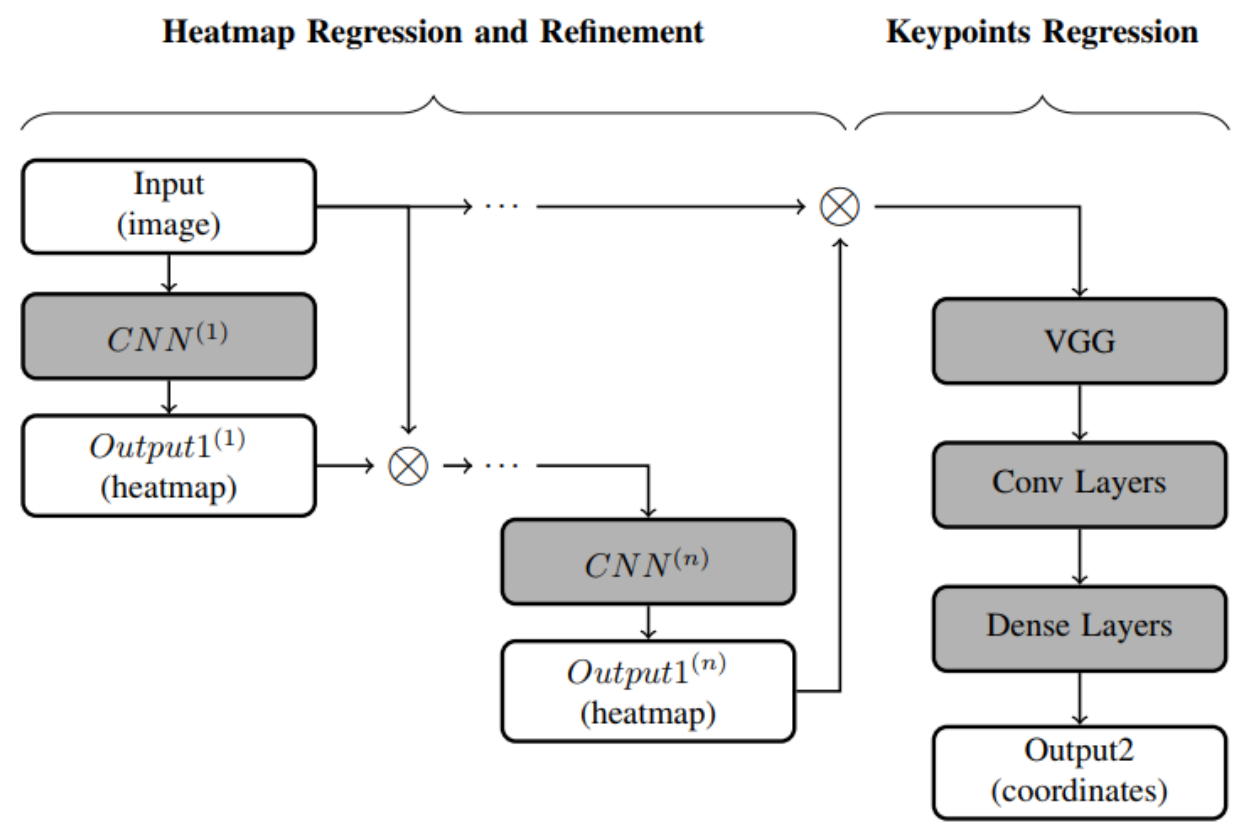

Figure 2.4: Proposed architecture for deep keypoint detection, from [6].

Regarding keypoint regression, the mean squared error, was the selected loss function. $N_{k}$ represents the number of coordinates, $x_{k}^{\text {target }}$ the ground truth for a single coordinate and $\hat{x}_{k}$ the prediction (Equation 2.2).

$$
\mathscr{L}_{\text {keypoints }}=\frac{1}{N_{k}} \sum_{\forall k}\left(x_{k}^{\text {target }}-\hat{x}_{k}\right)^{2}
$$

Mean squared error was also selected as loss function for the heatmaps regression. However, since heatmaps undergo an iterative process of refinement, the complete process is defined by Equation 2.3, where $N_{h}$ represents the number of heatmaps, $j$ represents a step in the refinement process and $\lambda_{j}$ represents the weight given to that step.

$$
\mathscr{L}_{\text {heatmaps }}=\sum_{j=1}^{N_{h}} \lambda_{j} \mathscr{L}_{\text {heatmap }}(j)
$$

The loss function for the heatmap in each step is defined in Equation 2.4, where $N_{p}$ corresponds to the number of pixels in the image, $x_{p}^{\text {target }}$ to the ground-truth and $\hat{x}$ to the prediction for the pixel values.

$$
\mathscr{L}_{\text {heatmap }}(j)=\frac{1}{N_{p}} \sum_{\forall p}\left(x_{p}^{\text {target }}-\hat{x}_{p}\right)^{2}
$$




\subsubsection{Available Datasets}

Currently, in order to perform experiments, there are three datasets: PORTO (Figure 2.5), with 120 images; VIENNA (Figure 2.6), with 71 images; TSIO (Figure 2.7), with 30 images. PORTO and TSIO datasets are composed of photographs obtained in similar conditions, which means that all the images belonging to these datasets have a distinct and consistent background. Also, the lighting conditions are quite good, i.e., photographs do not have many shadows nor artificial variations in colour. On the other hand, the VIENNA dataset was not obtained in ideal conditions,
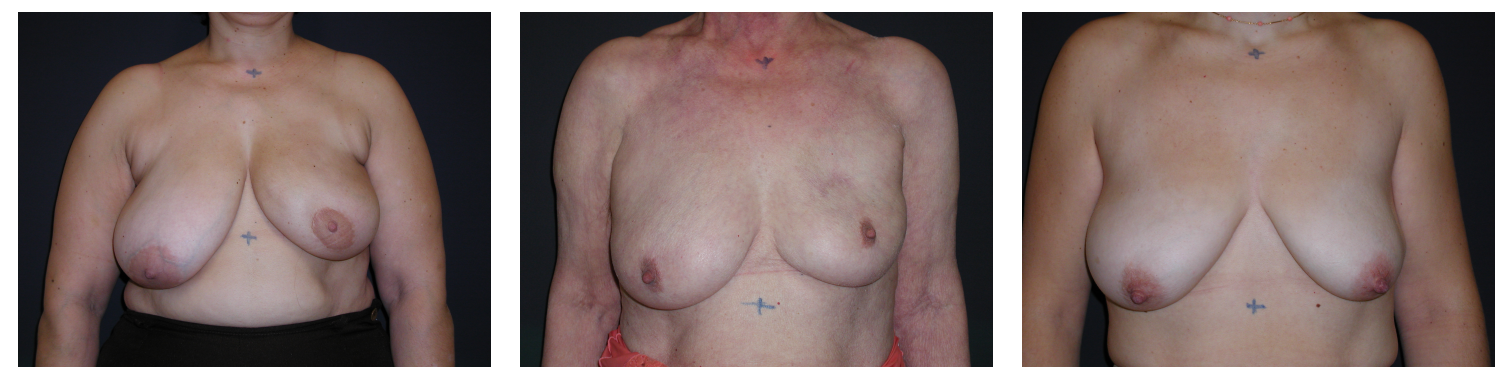

Figure 2.5: Example images from PORTO Dataset.
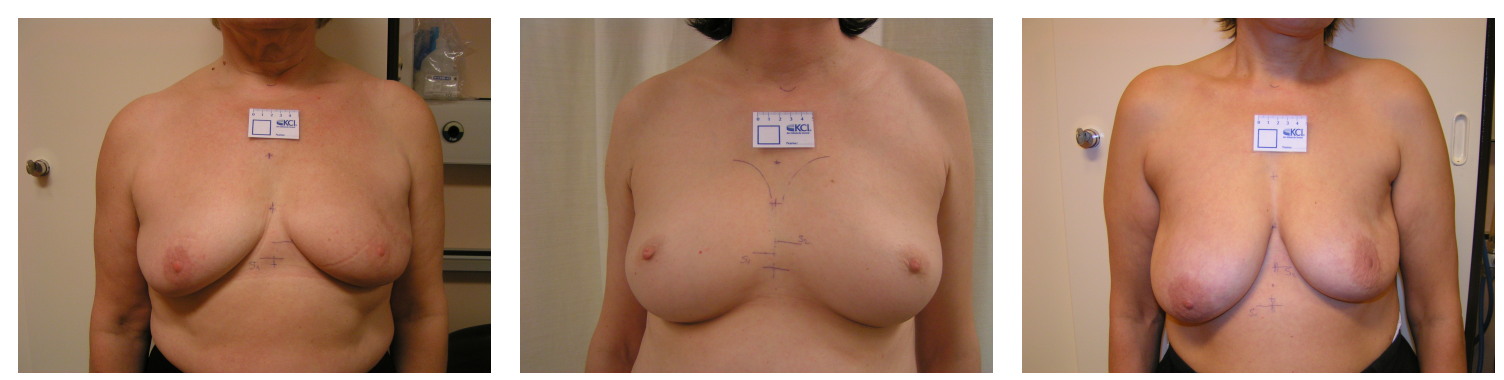

Figure 2.6: Example images from VIENNA Dataset.
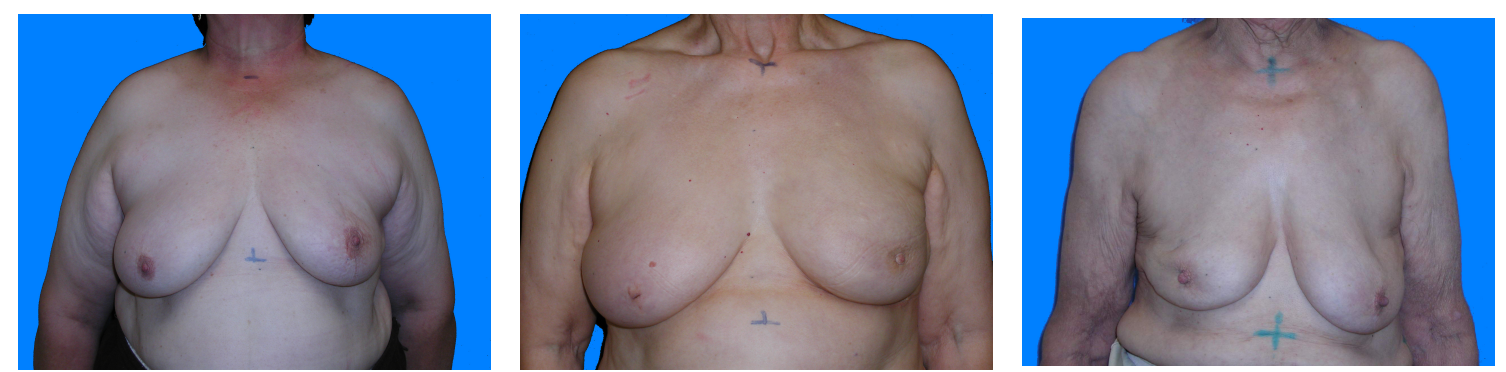

Figure 2.7: Example images from TSIO Dataset.

so, it is constituted of photographs where the background is variable and noisy, and the lighting conditions are poor. For each image, there are 37 ground-truth keypoints ( 4 endpoints, 30 points along the breast contours, 2 nipples and the supra-sternal notch); this means that there are available 74 coordinates, which can be used to train deep models. 


\subsubsection{Results}

Silva et al. [6] did a comparative study with three different models: traditional, DNN and hybrid. The traditional model is based on the methods described by Cardoso and Cardoso [75] and Cardoso et al. [82; 85], being referenced as the baseline method. The DNN model uses the methodology described in the previous Subsections and the hybrid model uses the endpoints detected by the DNN model and applies the shortest path algorithm [89] to detect the breast contour. Table 2.2 shows the results obtained by Silva et al., in [6], with the dataset described in the previous Subsection. As can be seen and as concluded by Silva et al. in the original work, the DNN model surpassed the baseline solution in all tasks but the breast contour, whereas the hybrid approach obtained the best results in terms of breast contour detection.

Table 2.2: Average error distance for endpoints, breast contours and nipples measured in pixels, from [6]. Best results are highlighted in bold.

\begin{tabular}{|c|c|c|c|c|c|c|c|c|c|}
\hline \multirow{2}{*}{ Model } & \multicolumn{3}{|c|}{ Endpoints } & \multicolumn{3}{|c|}{ Breast Contour } & \multicolumn{3}{|c|}{ Nipples } \\
\hline & Mean & Standard Deviation & Max & Mean & Standard Deviation & Max & Mean & Standard Deviation & Max \\
\hline Traditional model & 81 & 97 & 569 & 36 & 74 & 377 & 123 & 183 & 847 \\
\hline DNN model & 38 & 29 & 188 & 18 & 8 & 56 & 57 & 32 & 195 \\
\hline Hybrid model & 38 & 29 & 188 & 12 & 19 & 121 & 57 & 32 & 195 \\
\hline
\end{tabular}

\subsection{Conclusions}

The work described in Subsection 2.4, presented two important outcomes [6]:

1. A novel DNN model capable of improving state of the art algorithms in the detection of keypoints in photographs of women's torso, after being subjected to BCCT.

2. A hybrid model which uses the DNN model to detect endpoints, nipples and supra-sternal notch, and then finds the breast contour using the shortest path algorithm.

There is, however, space for work improvements, particularly regarding breast contour detection. In fact, in the original work, the hybrid model presents better results on the breast contour detection task (as can be seen in Table 2.2, both DNN and hybrid models achieve the same results in the endpoints and nipples detection tasks). This means that, if one aims to achieve a fully end-to-end deep learning method to perform classification of BCCT outcomes, it is mandatory to redefine the DNN model, so it can perform better than the hybrid model in all tasks. This can be achieved by different strategies (e.g., image segmentation or quality-driven approaches) which will be explored within this thesis. 


\section{Chapter 3}

\section{Image Segmentation for Breast Contour Detection}

Contents

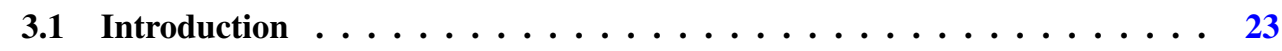

3.2 Traditional Approaches $\ldots \ldots \ldots \ldots \ldots \ldots \ldots \ldots \ldots$

3.3 Deep Learning Approaches $\ldots \ldots \ldots \ldots \ldots \ldots \ldots$

3.4 Segmentation for Breast Contour Detection . . . . . . . . . . . . 29

3.5 Implementation and Results . . . . . . . . . . . . . . . 29 29

3.6 Conclusions . . . . . . . . . . . . . . . . 31

\subsection{Introduction}

Image segmentation is the process of clustering an image into regions of interest (ROI), so that, every pixel belonging to that ROI is similar in terms of several characteristics (e.g. colour, texture, shape or intensity) [90; 91]. Typically, in biomedical applications, the employment of a proper segmentation method is of utmost importance, being one of the most relevant steps in the pipeline [92; 91]. With the advent of novel artificial intelligence techniques, it is possible to divide image segmentation into traditional or deep learning based approaches.

\subsection{Traditional Approaches}

Image segmentation algorithms within this group usually rely on domain knowledge and do not apply neural networks to fulfil this task. This also means that feature engineering techniques play an important role in the process and are relevant for the final result [93]. Although not being the focus of this thesis, it is important to recall that the common techniques under this scope are: thresholding [94; 95], edge based [96; 97], region based [98; 99; 100; 101], deformable models $[102 ; 101 ; 103]$ or graph cuts $[104 ; 105]$. 


\subsection{Deep Learning Approaches}

These type of approaches make use of the capabilities of DNNs to succeed in these tasks. Regarding the fact that one is dealing with images as input data, most of the state of the art architectures are based on convolutional neural networks (CNNs).

\subsubsection{Fully Convolutional Networks for Semantic Segmentation}

Following the high performance of CNNs in image-related tasks at a global (e.g., whole-image classification [106; 88; 107]) or local (e.g., bounding box object detection [108; 109; 110], part and keypoint prediction $[111 ; 112]$ or local correspondence $[111 ; 113])$ level, Long et al. have developed a fully convolutional network (FCN) which is trained end-to-end and/or pixel-to-pixel on semantic segmentation [7]. Generally, semantic segmentation presents two issues: global information, which is focused on the what, and local information, that is focused on the where. In order to resolve these two tasks, they designed an architecture that combines deep, coarse, semantic information and shallow, fine, appearance information. The intuition behind this approach is that it is possible to interpret classification CNNs as fully convolutional and then perform parameter fine-tuning from their learned representations; this can be understood as an extension of deep classification architectures, that uses image classification as a supervised pre-training, then fine-tuning at a fully convolutional level to learn from the whole-image inputs and whole-image ground-truths. Bearing in mind that a FCN operates on an input of any size and produces an output

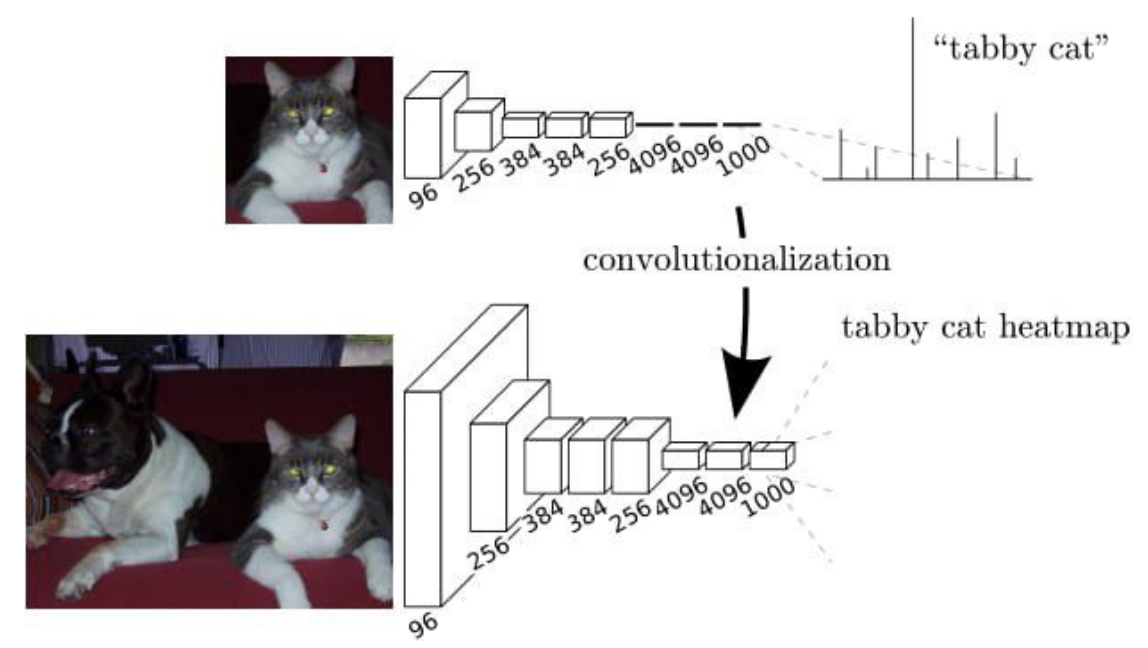

Figure 3.1: The concept of FCN, from [7].

of corresponding (probably resampled) spatial dimensions, it becomes crucial to understand how to turn classification DNNs into FCNs, thus, making them produce rough output maps. Also, since the main goal here is to reach pixelwise prediction, there is a need to connect those outputs back to the pixels. To convert a classification network into a fully convolutional one, it is necessary to 
look at the fully connected layers of the first. Within the original article, the authors use an example standard image recognition networks, such as LeNet [114] and AlexNet [106], which take fixed-sized inputs and produce nonspatial outputs; these networks' fully convolutional layers have fixed dimensions and discard spatial coordinates. On the other hand, it is possible to look at these as convolutions with kernels that cover their entire input regions; this will turn them into FCNs that can take inputs of arbitrary size and output classification maps (also known as heatmaps; see Figure 3.1). Due to the fact that FCNs are obtained from typical classification networks, the output maps usually have smaller dimensions than the inputs (i.e., they are reduced by subsampling), which will lead to a coarse output. To fix this issue, they perform an upsampling operation; in fact, they are connecting these rough outputs to dense pixels via interpolation. These concepts are of utmost relevance for the architectures explained in the following subsections, since they are based on them.

\subsubsection{U-Net}

In 2015, Ronneberger et al. proposed a new DNN for biomedical image segmentation: U-Net [8]. For them, there are two main problems with the typical usage of CNNs for biomedical image processing:

1. Besides a single image class label, the output should include localization (i.e., each pixel should be assigned a class label).

2. For biomedical tasks, a high number of images to train is usually hard to obtain.

Their starting point was the work developed by Ciresan et al. [115], which proposed a network in a sliding-window setup to predict the class label of each pixel by providing a local region (patch) around that pixel as input; their strategy presented two advantages: the network was able to localize and training data in patches is larger than the number of training images. On the other hand, this strategy also presented some problems: it was slow due to the fact that, for each patch, the network had to be run separately, larger patches required more max-pooling layers (which would lead to a decrease on localization accuracy) and small patches would allow the network to see only little context. Based on this, Ronneberger et al. decided to build their ideas upon the FCN [7]. Within their approach, they added successive layers with pooling operators replaced by upsampling operators to the baseline network, in order to increase the resolution of the output. Also, the upsampling part has a large number of feature channels, which allow the network to propagate context information to higher resolution layers. Also, U-Net does not have fully connected layers and only uses the valid part of each convolution (the segmentation map only contains the pixels) for which the full context of the image is available in the input image. Figure 3.2 shows the architecture proposed by Ronneberger et al.

\subsubsection{Global Convolutional Network}

Proposed by Peng et al. in 2017, this work addresses two challenges: 


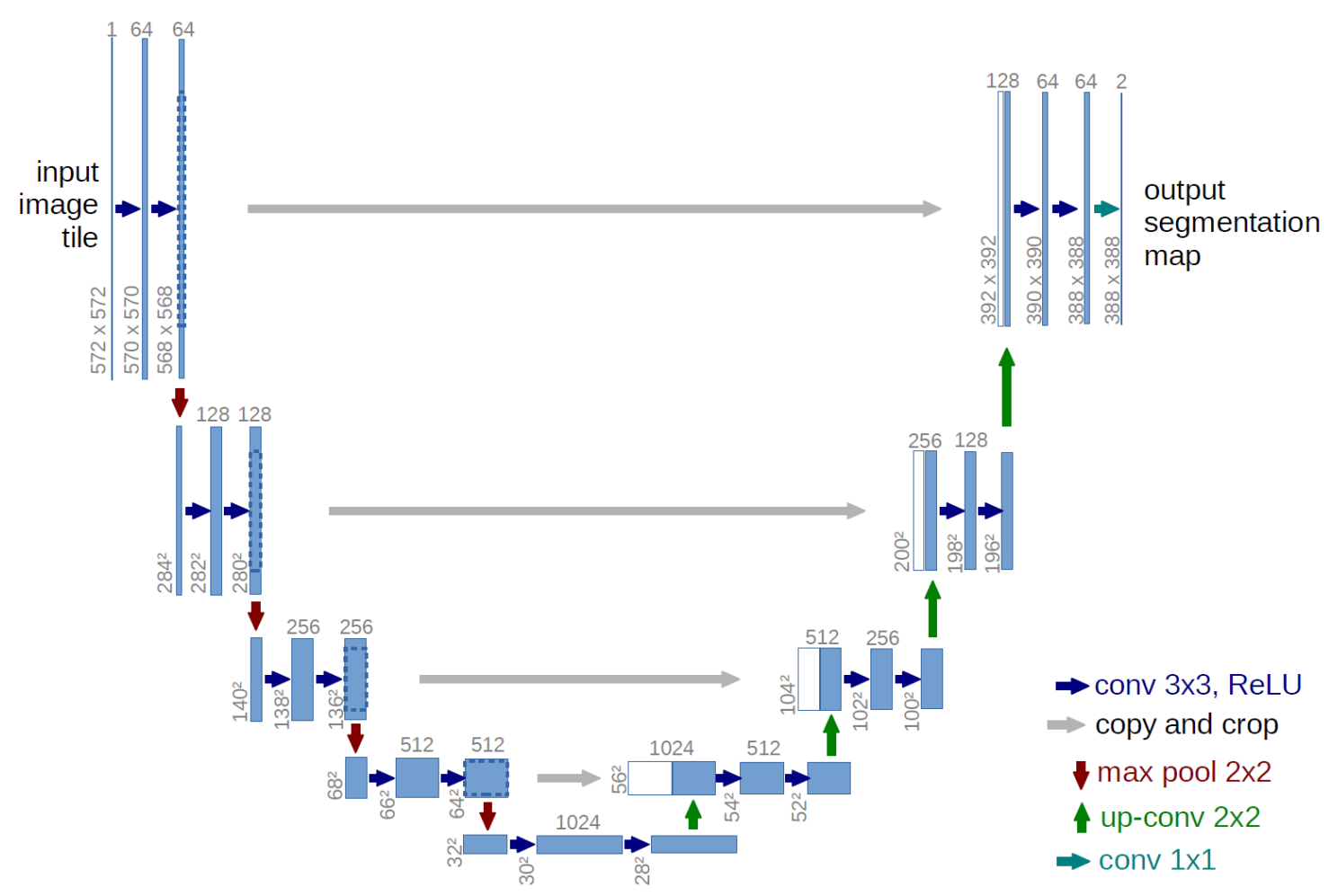

Figure 3.2: U-Net architecture, from [8].

1. An object associated with a specific semantic concept should be labelled correctly (i.e., a classification problem).

2. The classification label for a pixel must be aligned with the appropriate coordinates in the output score map (i.e., a localization problem).

The main difficulty here is that these two challenges are antagonistic. For instance, for a classification problem, it is expected that the model is invariant to several transformations, such as translation and rotation; on the other hand, when dealing with a localization problem, the model should be sensitive to that same transformations, so it can accurately locate every pixel for each semantic category. In fact, most of the semantic segmentation algorithms aim to solve the localization problem, which may jeopardize the performance on the classification task [9]. To overcome these issues, the authors have proposed a novel architecture called Global Convolutional Network (GCN), which is able to simultaneously deal with the two issues. To achieve a fully working model, they use a FCN-like structure as their basic framework and guide their work through two fundamental design principles:

1. To address the localization challenge, the model structure needs to be fully convolutional, so it can retain the localization performance, and no fully-connected or global pooling layers may be used, due to the fact that they will discard localization information. 
2. On behalf of the classification task, it is necessary to adopt a large kernel size in the network architecture in order to enable densely connections between feature maps and per-pixel classifiers, leading, thus, to an enhancement in the ability to handle different transformations.

Based on these guidelines, the authors have proposed a network with two modules: GCN, which is responsible for solving the classification and localization problems at the same time; and a boundary refinement module that aims to improve localization performance near object boundaries. Commonly, deep learning models present significant differences, which are directly related to their principal task. Regarding classification architectures, features are usually extracted from a hidden layer which is rough on spatial dimensions, and classifiers are densely connected to the entire feature map through a fully-connected layer [116; 117] or a global pooling layer [107; 118; 119], generating, thus, features that are invariant to local perturbations and allowing classifiers to handle different types of input transformations. On the other hand, for localization purposes, there is a necessity of large (i.e., with high-resolution) feature maps to encode more spatial information; these are usually generated with specific techniques such as deconvolution [7], unpooling [120; 121] or dilated-convolution $[122 ; 123]$. In this case, classifiers are locally connected to each spatial location on the feature map to generate pixel-wise semantic labels [9]. In order to overcome both
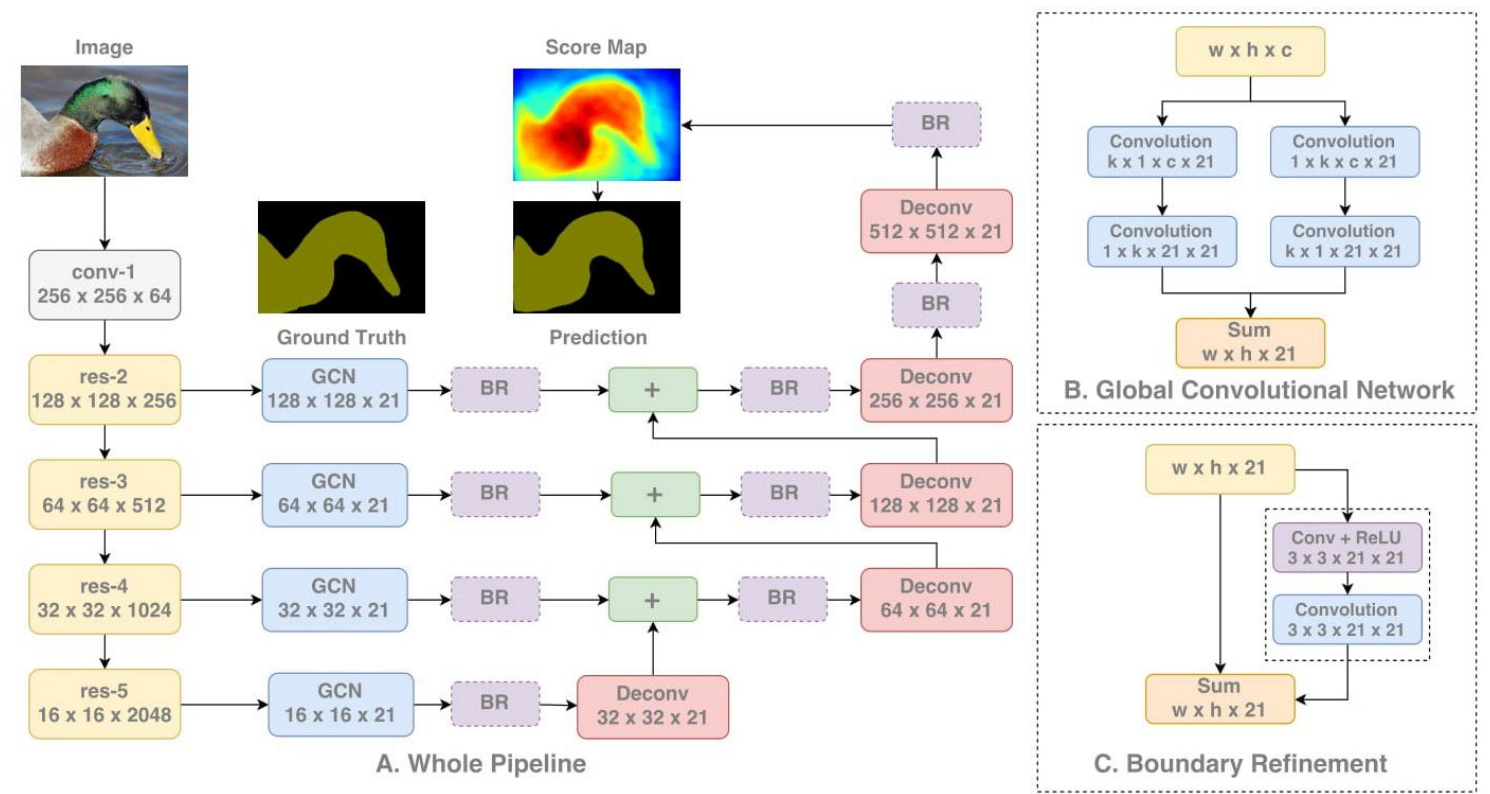

Figure 3.3: GCN architecture, from [9].

problems, a new architecture was designed. Besides GCN and boundary refinement modules, this architecture relies on ResNet [119] for feature extraction and on FCN4 for segmentation [7; 124]. Regarding workflow (see Figure 3.3), multi-scale feature maps are initially extracted with the ResNet backbone and then, GCN blocks generate multi-scale semantic score maps for each class. One may also notice that, in several intermediate steps, the boundary refinement structure is employed in order to improve the localization performance near object boundaries. 


\subsubsection{DeepLabv3+}

Published by Chen et al. in 2018, DeepLabv3+ (see Figure 3.4) is, in fact, an extension of DeepLabv3 [125], with the addition of an encoder-decoder module to refine segmentation results. This work is based on the use of atrous convolution, or dilated convolution, motivated by one issue related to the use of CNNs: the use of consecutive pooling operations, or convolution striding, make CNNs able to learn a large variety of abstract representations, but, on the other hand, such invariance to local image transformation could prevent dense prediction tasks, where detailed spatial information is desired. With atrous convolution, it is possible to manipulate the resolution at which feature responses are computed, without the need of learning extra parameters; this is related with the fact that this technique removes the downsampling operations from the last few layers and performs an upsampling to the corresponding filter kernels, creating, thus, some kind of holes, between filter weights. Important to notice that, since there are other versions of DeepLab [126; 127], it is relevant to retain other fundamental techniques which contribute to this network performance. For instance, the usage of spatial pyramid pooling, employed in DeepLabv2 [127] as atrous spa-

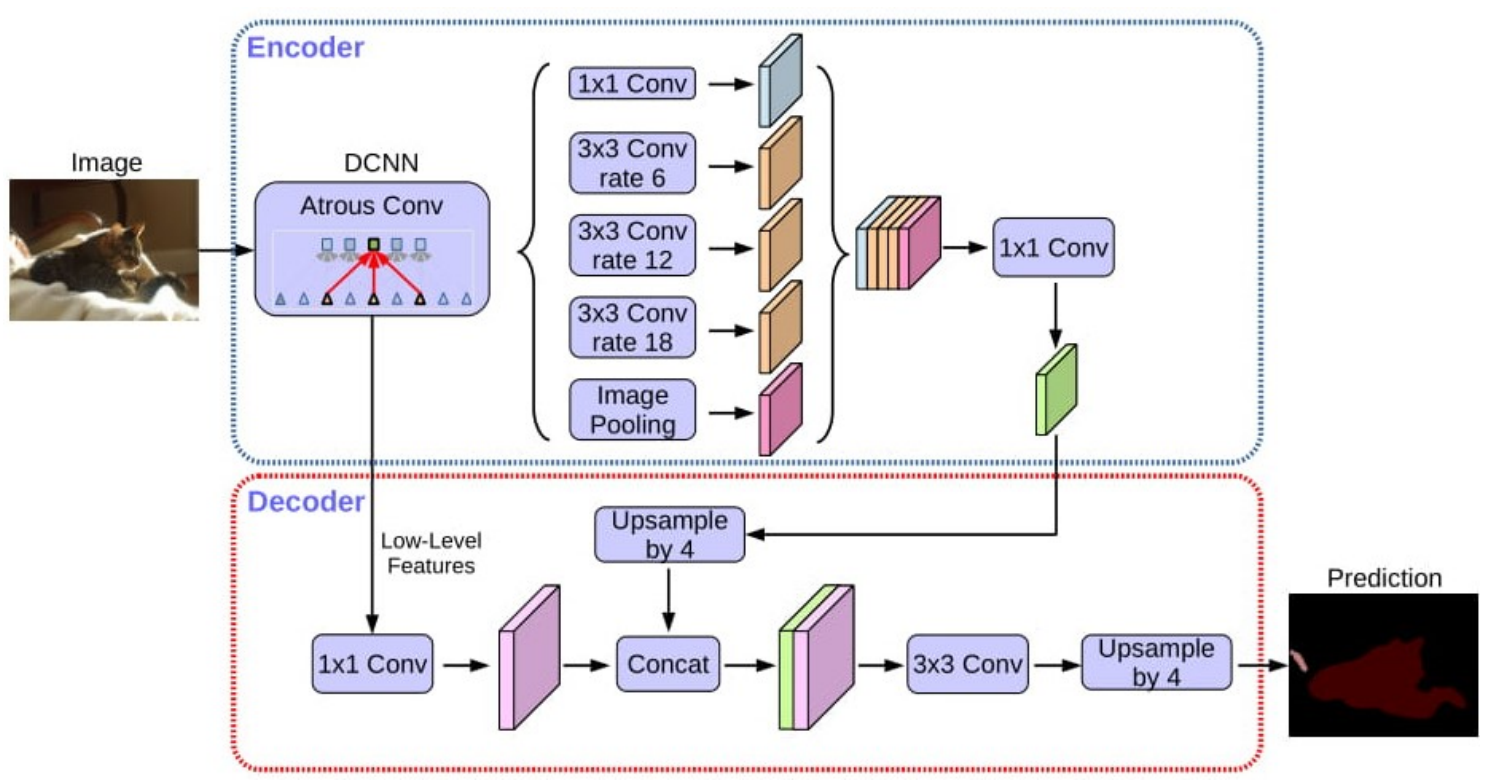

Figure 3.4: DeepLabv3+ architecture, from [10].

tial pyramid pooling (ASPP), to capture multi-scale information; there are similar works on this field that deserve some analysis, such as ParseNet [128] for global context information or Pyramid Scene Parsing (PSP) Net, in which spatial pooling is performed at several grid scales, thus, demonstrating state of the art performance in semantic segmentation [129]. Another important detail is the encoder-decoder structure, also used by benchmark networks on segmentation, such as SegNet [130] or U-Net [131]; within the encoder, spatial dimension of feature maps is gradually reduced leading to a facilitated capture of longer range information in the deeper encoder output, whereas, in the decoder, object details and spatial dimension are gradually recovered. Taking into account these informations, DeepLabv3+ is an improvement of DeepLabv3 where: an 
encoder-decoder with atrous convolution is designed which allows the arbitrary control of the resolution of the extracted encoder features; the Xception [132] model is integrated as backbone for the segmentation task and depthwise separable convolution is applied to both ASPP and decoder modules [10].

\subsection{Segmentation for Breast Contour Detection}

The main hypothesis behind this approach is that it should be easier to detect breast contours if one is able to properly detect the breast first. As can be noticed, this is a challenge of semantic segmentation, where one needs to properly separate both breasts (i.e., foreground) from the other image components (i.e., background). In this case, the segmentation task would work as means to an end; ideally, within this approach, the pipeline would be composed of two main processes: breast segmentation and breast contour detection, where the second is fully-dependent on the first. In this experience, the main goal was to study the capabilities of state of the art segmentation DNNs on the specific task of segmenting both breasts from the images of the dataset (see Chapter 2). It is important to take into account that one can only move to the subtask of breast contour detection if good performance is achieved in the segmentation task. In the next section, methodology and results are presented.

\subsection{Implementation and Results}

For this study, it was decided to use state of the art architectures, such as: U-Net, GCN and DeepLabv3+. The dataset (221 images) was divided into train (107 images), validation (47 images) and test (67 images) sets. All images were first resized to the dimensions of $512 \times 384$. Ground-truth masks were obtained with support of the ground-truth keypoints and images: every pixel inside the keypoints' area was given the 255 value; the rest of the pixels was given the 0 value. Regarding the performance metric, for these experiences, it was used the Dice Coefficient [133] (DC), which is very common in image segmentation tasks; DC values near 1 mean that there is high similarity between both ground-truth and predicted masks. It can be written as

$$
D C=\frac{2 \sum_{i}^{N} p_{i} g_{i}}{\sum_{i}^{N} p_{i}^{2}+\sum_{i}^{N} g_{i}^{2}}
$$

where $N$ represents the total number of pixels, $p_{i}$ is the predicted pixel label, $g_{i}$ is the ground-truth pixel label and $i \in\{0,1\}$, meaning that this is a binary-class problem.

Regarding loss function, the Dice Coefficient Loss (DCL) was chosen and monitored during training. It can be written as

$$
D C L=1-D C
$$


where $D C$ is the Dice Coefficient.

U-Net was implemented in Keras [134] and GCN was implemented in PyTorch [135]. Both were trained during 300 epochs, with Adadelta [136] as optimization function. During training phase, data augmentation techniques such as rotation, horizontal and vertical shifting, shear mapping, zoom in and zoom out or horizontal and vertical flips were employed. The model with the lower loss value on the validation set was chosen during training and saved. It was then used to perform inference and evaluation on the test set. Results on test set are shown in Table 3.2; each DC value on the table is actually the average DC value obtained with each model in test set. Due to the lack of computational resources it was not possible to train the TensorFlow implementation of DeepLabv3+. Table 3.1 shows mask predictions of U-Net and GCN respectively compared against the ground-truth masks.

Table 3.1: Examples of masks obtained with GCN and U-Net. Results are divided in good or bad, in comparison with the ground-truth masks.

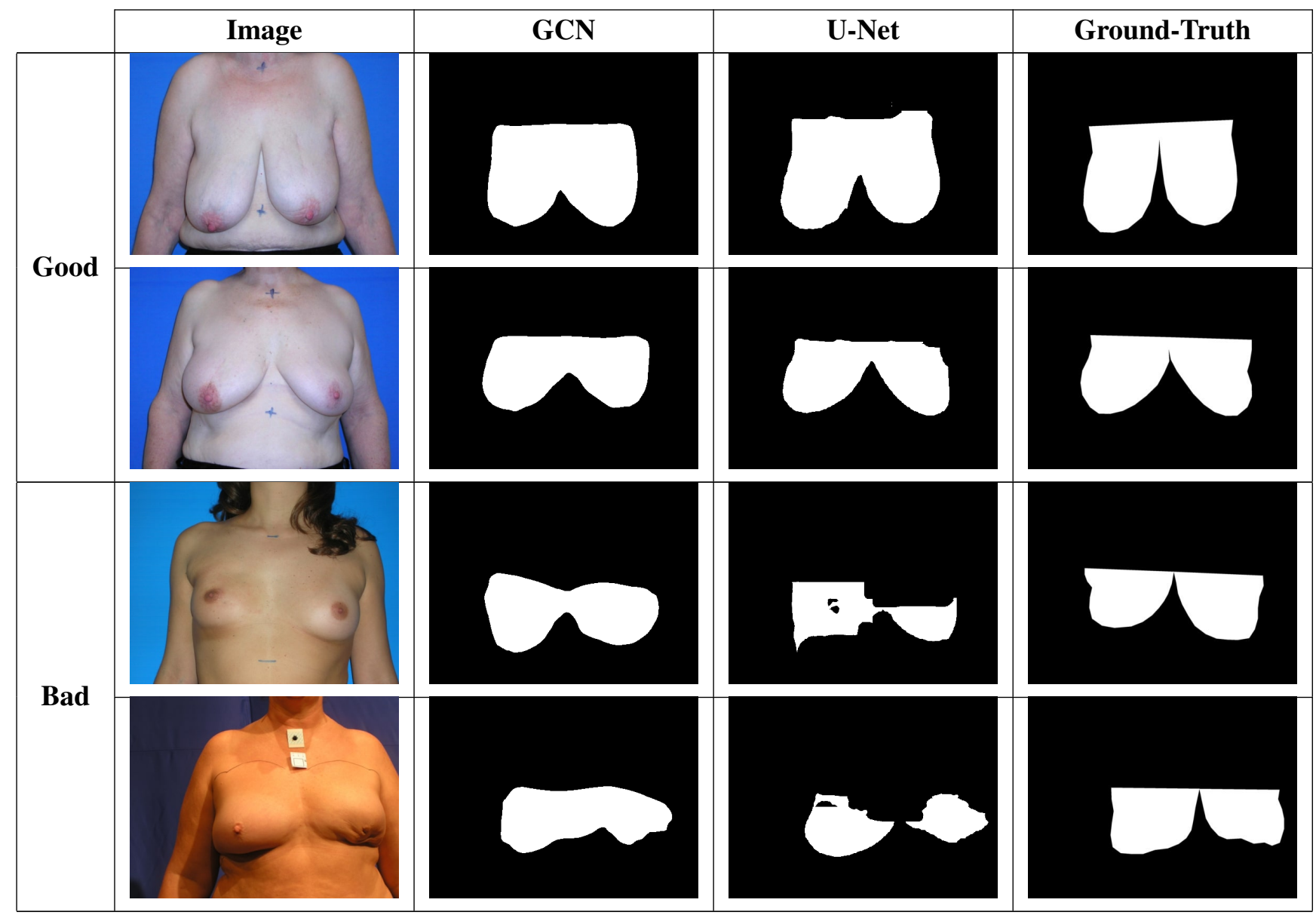


Table 3.2: Average Dice Coefficient results on test set for U-Net and GCN models. Best result is highlighted in bold.

\begin{tabular}{|c|c|}
\cline { 2 - 2 } \multicolumn{1}{c|}{} & Average Dice Coefficient \\
\hline U-Net & 0.8689 \\
\hline GCN & $\mathbf{0 . 8 9 3 7}$ \\
\hline
\end{tabular}

\subsection{Conclusions}

Although it was possible to obtain DC results near 1, for this specific task, they do not contribute as much as it was initially expected. Here, the main goal was to generate breast binary masks from a given image in order to detect breast contours, being a two-class segmentation problem, where breasts are foreground and the rest of the image is the background. Taking this into account, it is possible to explain high results on the DC score: masks are mostly composed by background pixels (imbalanced-class problem), so it is easier for the network to predict background instead of foreground due to the fact that it has naturally seen more background pixels, during training. On the other hand, it can be said that, during inference, the trained models are able to predict the most probable breast localization. The main issue is that this is still a fuzzy localization, which means that it will not help in finding breast contours. Also, when images contain small or undefined breasts, it becomes even harder to obtain reliable breast image masks, which would lead to increased difficulty in finding breast contours. For these reasons, post-processing experiences to detect contours after segmentation were not performed. On the other hand, since breast contour detection fully depends on excellent results on image segmentation, this approach, at the moment, is not a viable option. 


\section{Chapter 4}

\section{Quality-Driven Keypoint Detection}

\section{Contents}

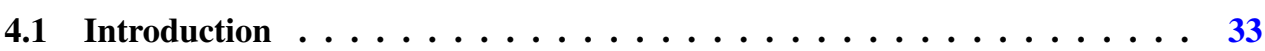

4.2 State of the Art on Deep Quality-Driven Architectures . . . . . . . . . . 33

4.3 Quality-Driven Architectures for Keypoint Detection . . . . . . . . . 37

4.4 Implementation and Results $\ldots \ldots \ldots \ldots \ldots$

4.5 Conclusions . . . . . . . . . . . . . . . . 46

\subsection{Introduction}

As stated in Chapter 2, breast contours can be modelled with an active contour based on splines with control points (i.e., keypoints). State of the art methodology on keypoint detection was established by Silva et al. [6] and is based on the use of a novel DNN model and on a hybrid model. However, they are still open to further improvements on the breast contour detection task. Taking this into account, two deep quality-driven keypoint detection methodologies are proposed and discussed in the following sections.

\subsection{State of the Art on Deep Quality-Driven Architectures}

Generally, deep learning architectures for image analysis take an image as input and return a mask (image segmentation) or a value (image classification) as output; these deep models are then regularized in a supervised manner on the object of interest. On the other hand, the need for great amounts of training data or the necessity of re-training/fine-tuning such models in order to apply them in different contexts may be problematic [11]. Taking this into account, Fernandes et al. have proposed a different methodology, based on two fundamental concepts:

1. In their daily tasks, humans usually have different possible solutions available, instead of relying on a single ground-truth. This choice usually involves some kind of utility function that reflects a degree of satisfaction with a certain solution [137]. 
2. The use of an iterative refinement method to improve the quality of the desired object, based on the backpropagation algorithm, is more robust than traditional inference methods.

\subsubsection{Deep Image Segmentation by Quality Inference}

In their original work, Fernandes et al. explored these concepts on image segmentation [11]. Initially, they started by defining the quality metric for the learning phase; they ended up choosing DC, which is widely used in this type of tasks as a similarity metric. Considering that modelling of utility functions is usually inspired in pairwise preferences [138] and/or cardinal/ordinal functions [139], the authors proposed a model that is capable of learning the quality (i.e., DC), given an image and mask pair; in this case, the utility function is precisely this measure of correspondence between a mask and an image. To achieve such a model, the authors actually discuss two possible straightforward solutions: using a conventional CNN, where the mask is appended to the image as an additional channel, or having two separate streams for the input image and masks, which are then merged in the last dense blocks by concatenating their latent representation; both of them presented obstacles that would difficult the training phase [11], so the authors had to come up with a different proposal. Their deep architecture, Gossip Network, receives an image and mask pair as input and has two streams that try to model the foreground and background, respectively by the input mask. Moreover, as a strategy to increase/decrease the network's confidence in the recognition of their corresponding regions, these streams communicate (i.e., to gossip) between each other. Figure 4.1, Figure 4.2 and Figure 4.3, show the schemes of the Gossip Network architecture, its stream modules and its gossip blocks, respectively.

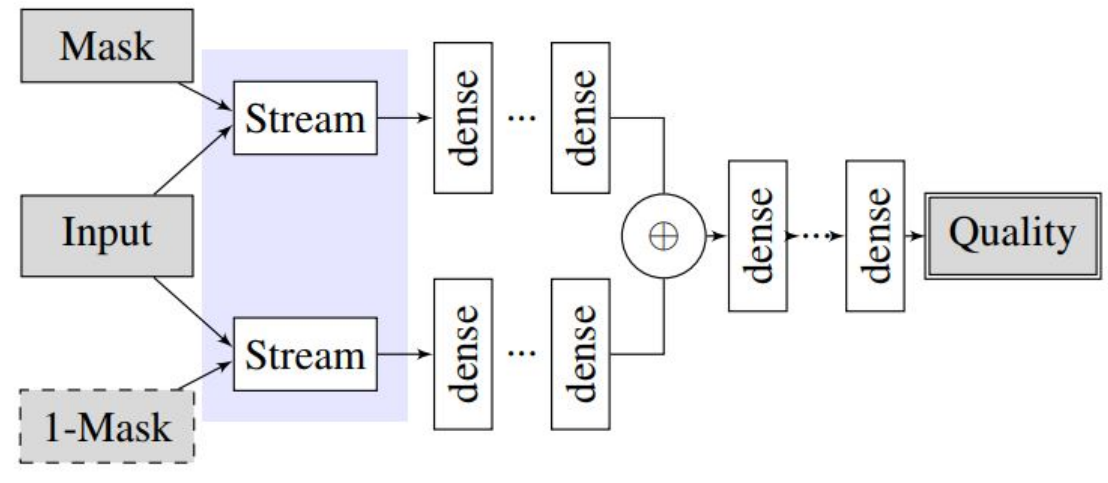

Figure 4.1: Gossip Network architecture, from [11]. Note that 1-Mask is the complement of the Mask.

After training, the Gossip Network is used to predict the quality of a given image and mask pair and, based on this prediction, the mask is iteratively refined by backpropagation until it reaches a stop criteria (e.g., quality value, number of iterations). To understand the application of this concept on the refinement of predicted masks, one must recall its usage in the proper training of DNNs. Assuming that $\mathscr{L}$ represents the loss function to be minimized, $t$ represents the target, $\hat{t}$ 


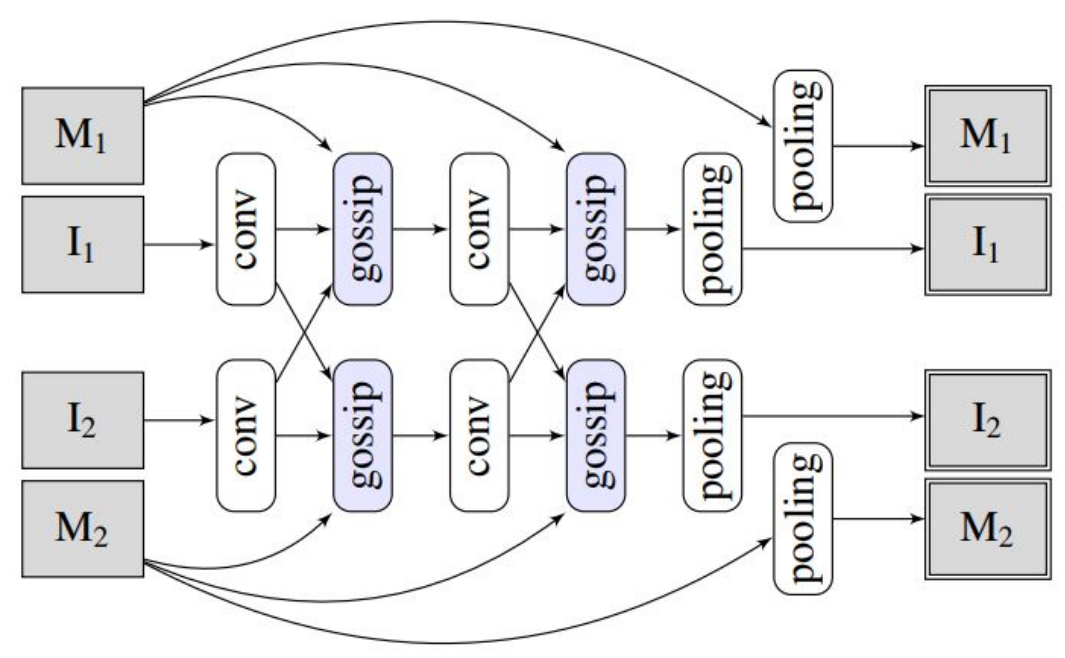

Figure 4.2: Stream module of the Gossip Network architecture, from [11].

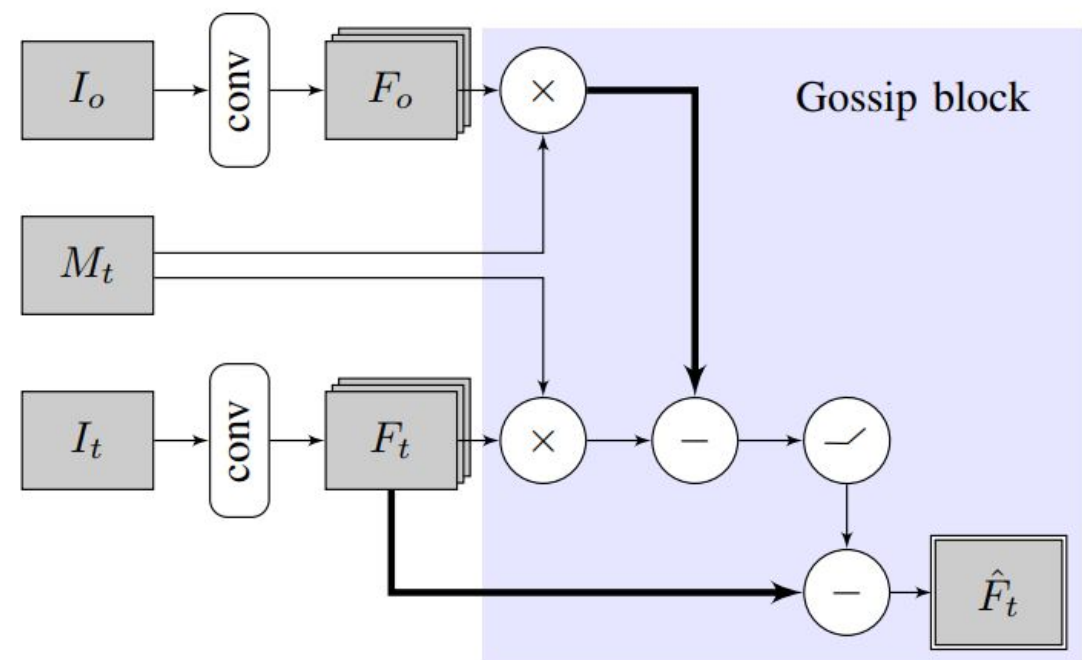

Figure 4.3: Gossip block of the Gossip Network architecture, from [11].

represents the prediction and $w$ represents an arbitrary weight to be learned/updated, the gradient of the loss function with respect to the weight can be computed as:

$$
\frac{\partial \mathscr{L}(\hat{t}, t)}{\partial w}
$$

The weight is then updated by gradient descent (i.e., the opposite direction of the gradient), with a learning rate of $\alpha$ as follows:

$$
w \leftarrow w-\alpha \frac{\partial \mathscr{L}(\hat{t}, t)}{\partial w}
$$


This is known as backpropagation. In order to apply such a concept in the refinement of segmentation masks, one just needs to change Equation 4.2, so that it becomes:

$$
\hat{m} \leftarrow \hat{m}+\alpha \frac{\partial \hat{q}}{\partial \hat{m}}
$$

where $\hat{m}$ represents the initially predicted mask (also referred to as initial mask candidate) and $\hat{q}$ is the predicted quality, obtained by inference with the Gossip Network. With this method, one is performing backpropagation on the predicted mask $\hat{m}$ by maximizing the predicted quality $\hat{q}$, with gradient ascent (important to notice that in this case, one is computing the gradient of predicted $\hat{q}$ with respect to the predicted mask $\hat{m}$ ). With this methodology, the authors were able to improve existing segmentation and create new segmentations from scratch (by starting with a black mask).

\subsubsection{Quality-based Regularization for Iterative Deep Image Segmentation}

Recently, Rebelo et al. have presented a new methodology, which uses the notion of quality as a regularization method during the training of a network for direct segmentation refinement [12]. They proposed a network with an encoder-decoder structure which takes an image and a mask as inputs, and returns quality and a refined segmentation as output, following a multi-task learning paradigm. Although this work follows a similar strategy to the one presented by Fernandes et

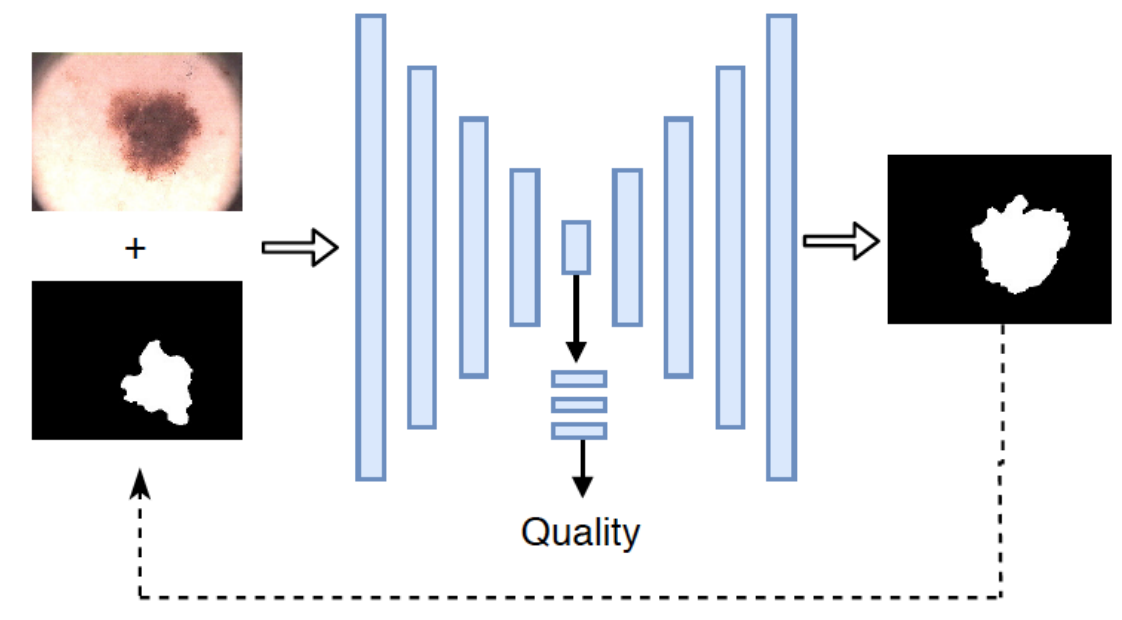

Figure 4.4: Iterative segmentation refinement network architecture (with skin lesion image as example), from [12].

al. [11] (e.g., the quality metric is the same), the main focus in this case was the study of a DNN which could receive the predicted mask from a state of the art network and iteratively improve the segmentation, while predicting the quality of the input segmentation, at the same time; this parallel quality prediction can be understood as a regularization method that will favour the learning of features connected to the degree of correction necessary to improve the input segmentation. Figure 4.4 shows the proposed network architecture. To start the training, it is required to feed the 


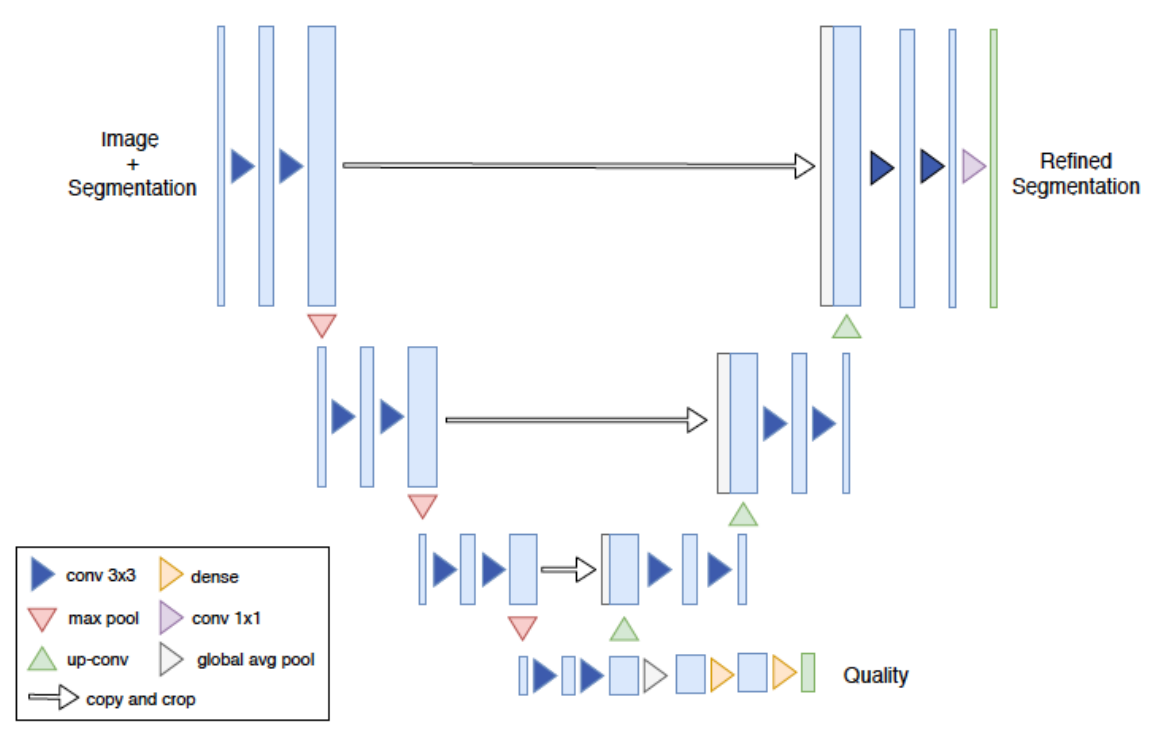

Figure 4.5: U-Net [8] with quality prediction module, from [12].

network with an image and an initial segmentation mask (obtained from any other model); this initial mask will be refined according to an iterative method, where the network will use its own output as a new input for further improvement, leading, thus, to higher predicted quality values. In their original work, Rebelo et al. use U-Net [8], trained on the same dataset, to obtain the initial segmentation masks, which are then concatenated to the input image as an additional channel. As can be seen in Figure 4.5, the quality prediction module was added as an extension at the embedding of U-Net, allowing it to use the information extracted by the encoder, before the decoding process [12].

\subsection{Quality-Driven Architectures for Keypoint Detection}

Based on the works of Fernandes et al. and Rebelo et al., two different methodologies for qualitybased keypoint detection are proposed and discussed.

\subsubsection{Deep Keypoint Detection by Quality Inference}

The main idea behind this first approach is the application of the main concepts from [11] to keypoint detection, instead of image segmentation. To achieve such a model, one must first define a suitable quality metric. Bearing in mind that the main task here is keypoint coordinate regression and following the work developed in [6], mean squared error (MSE) was chosen as the quality metric. It is written as:

$$
M S E=\frac{1}{n} \sum_{i=0}^{n-1}\left(k_{i}-\hat{k}_{i}\right)^{2}
$$


where $n$ is the number of samples (i.e., number of coordinates in each keypoints array), $i$ is the index of the value, $\hat{k}$ is the predicted keypoints array and $k$ is the ground-truth keypoints array. The main objective is to have a model that receives an image and an array with initial keypoints candidates as inputs and that outputs a quality value (i.e., MSE between the candidate keypoints array and the ground-truth keypoints array). Figure 4.6 shows the proposed architecture. The image is given as an input to a CNN, a latent representation is obtained and then concatenated with the candidate keypoints array. This concatenation is then fed to a Multilayer Perceptron (MLP) which will output the quality value predicted, related to the image and the candidate keypoints. As

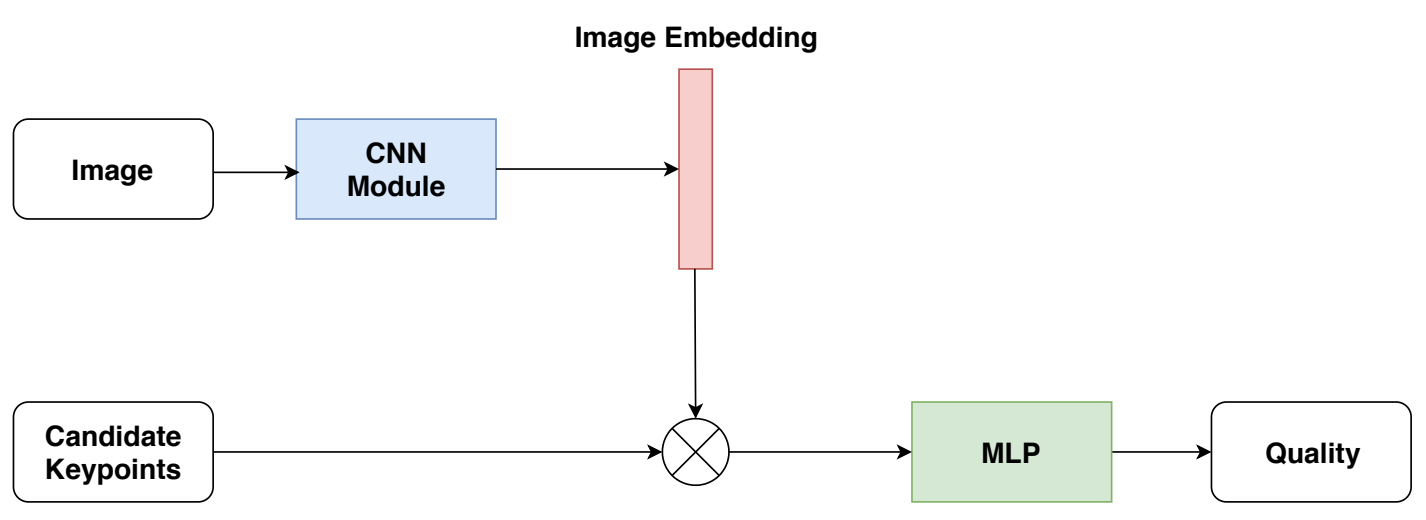

Figure 4.6: Keypoint's quality inference network architecture.

shown in Figure 4.7, the CNN Module is composed by VGG16 [88] as backbone without its dense layers, followed by four convolutional layers and three dense layers; the latent representation of the image is obtained from the last dense layer. This latent representation is then concatenated with the candidate keypoints array which is then fed to a MLP; this MLP has three dense layers.

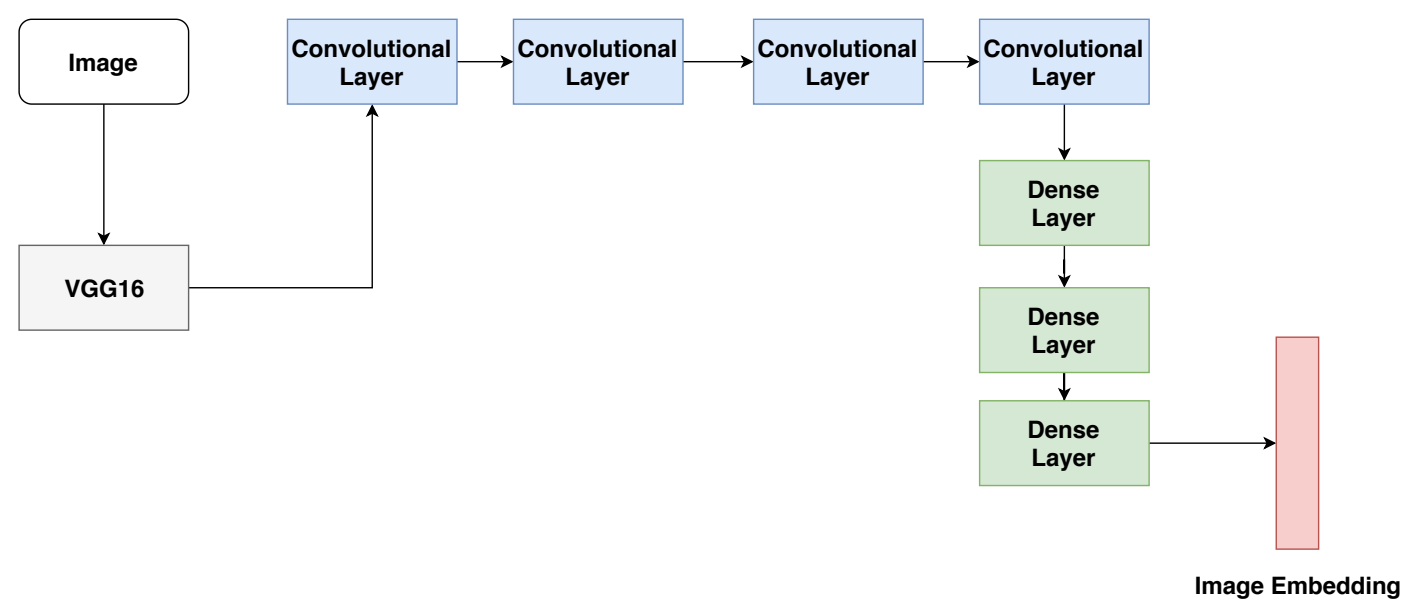

Figure 4.7: CNN Module's architecture.

The quality value is obtained from the last dense layer, as shown in Figure 4.8. After training the model on the quality inference task, the next step would be the use of the backpropagation 


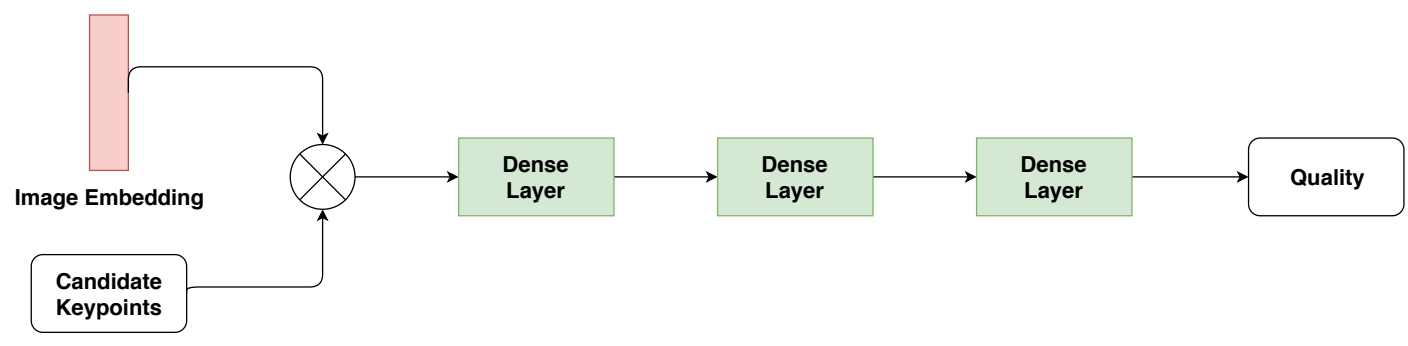

Figure 4.8: MLP's architecture.

algorithm to improve the predicted quality, leading, thus, to coordinate values near ground-truth. In order to do this, one must first to compute the gradients of the predicted quality, $\hat{q}$, with respect to the given candidate keypoints, $k^{\prime}$, and use gradient descent (important to remind that the quality metric is given by an error metric, so, what is aimed is the minimization of this value) with a learning rate, $\alpha$, as follows:

$$
k^{\prime} \leftarrow k^{\prime}-\alpha \frac{\partial \hat{q}}{\partial k^{\prime}}
$$

After several steps, it would be expected that the real MSE between the changed candidate keypoints and the ground-truth keypoints would be lower than the real MSE between the initial candidate keypoints and the ground-truth keypoints.

\subsubsection{Deep Iterative Refinement of Keypoint Detection}

This second approach aims to apply the methodology proposed in [12] to the task of keypoint detection, through the iterative refinement of the given candidate keypoints. The proposed architecture can be seen in Figure 4.9. Similarly to the work developed by Rebelo et al., the main objective here is to use an iterative approach to improve the quality (in this case, minimize its value, since it is a MSE value) of the given candidate keypoints; the quality value acts here as a form of regularization, during training. When compared to the architecture presented in Subsection 4.3.1, this one has some modifications. The CNN Module keeps the same structure, however, three new MLPs are added to the model in order to fulfil the main objective. The Intermediate MLP receives as input the result of the concatenation between the candidate keypoints and the Image Embedding and generates what is called an Intermediate Embedding, which has lower dimensions than the first one. This latent representation is then given to the Keypoints MLP, which has the task of predicting the keypoints coordinates, and to the Quality MLP, which has to learn how to predict the quality value, related to the image and candidate keypoints pair. In other words, the model is designed to be trained in a multi-task setting. 


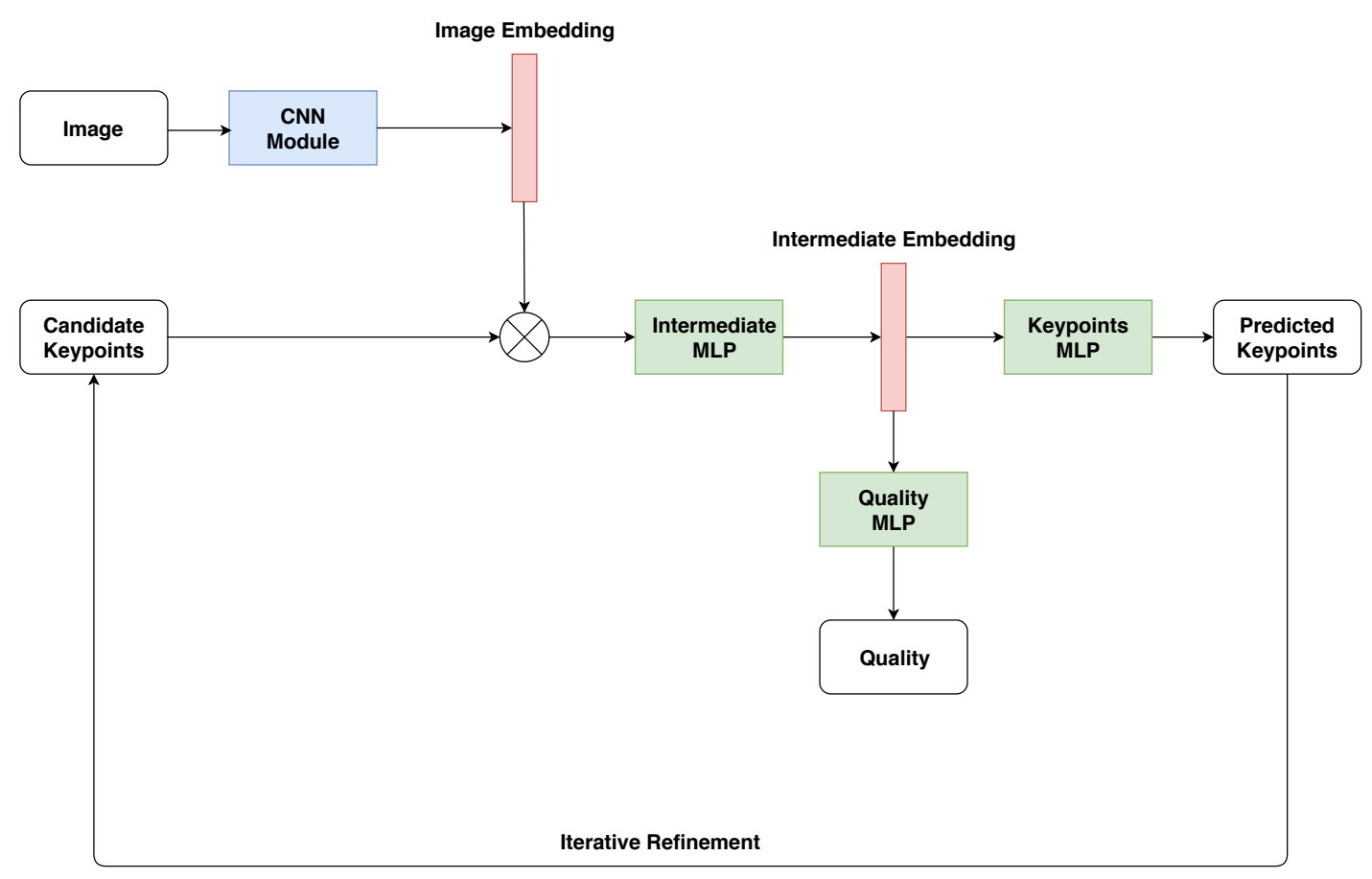

Figure 4.9: Proposed architecture for the iterative keypoint refinement network.

\subsection{Implementation and Results}

In the following Subsections, details related to the implementation of each of the different proposals are explained.

\subsubsection{Deep Keypoint Detection by Quality Inference}

This model was implemented in Keras [134]. Regarding data preparation, all images were first resized to the dimensions of $512 \times 384$; keypoints were also resized accordingly and were normalized by the width of the image, in order to be between 0 and 1 . The dataset was divided into train, validation and test sets, as in Chapter 3. To ensure that the model was robust, data augmentation techniques were employed during training, in an online fashion, to both images and ground-truth keypoints: translations, rotations and flips were used. Since the main objective is to teach the model on quality inference, it is important to ensure that a large range of quality values is shown to the network, during training; in order to achieve that, one must be certain that to the initial candidate keypoints were applied the correct diversity of transformations, so that, the correspondent qualities cover a wide range of values. To do this, the data augmentation strategy presented by Fernandes et al. in [11] was adapted and used; in this case, the transformations that were applied to the keypoints were translations, rotations and horizontal flips. Once again, all candidate keypoints and their correspondent qualities are generated in an online setting to keep dynamic training. Regarding the training phase, both image embedding (regularization term) and quality (main goal) 
were supervised, i.e., the loss function was defined as follows:

$$
\mathscr{L}_{\text {model }}=\lambda_{1} \mathscr{L}_{\text {image embedding }}+\lambda_{2} \mathscr{L}_{\text {quality }}
$$

where $\lambda_{1}$ and $\lambda_{2}$ represent non-negative weights attributed to each loss. The loss of the image embedding is computed as the MSE between predicted image embedding, $b$ and the ground-truth keypoints, $k$ :

$$
\mathscr{L}_{\text {image embedding }}=\operatorname{MSE}(b, k)
$$

The intuition behind this supervised approach is to guide the CNN Module to learn a latent representation that is near the ground-truth keypoints in order to facilitate the learning of the quality by the MLP. Regarding quality prediction, the loss function is also computed as the MSE between the predicted quality value, $\hat{q}$, and the ground-truth quality, $q$, for the candidate keypoints:

$$
\mathscr{L}_{\text {quality }}=\operatorname{MSE}(\hat{q}, q)
$$

The model was trained during 300 epochs, with Adadelta [136] as optimization function, and the model with lower loss on the validation set was saved. Table 4.1 and Table 4.2 present the layer's hyperparameters that were used in the CNN Module and MLP, respectively. After training, several initial candidate keypoints for test set were generated, following three possible situations: candidate keypoints were the result of the prediction obtained with the model described in [6] in images of the test set; candidate keypoints were the result of random generated values; candidate keypoints were coordinates with value 0 . The quality was then inferred from the initial candidate keypoints, using the trained model, and then the backpropagation algorithm proposed was applied. Regarding backpropagation, the presented results use a learning rate $\alpha=0.001$, selected taking

Table 4.1: CNN Module layer's hyperparameters (VGG16 not included).

\begin{tabular}{|c|c|c|c|c|c|c|}
\hline Layer Number & Type & Number of Neurons & Number of Filters & Filter Size & Activation Function & Dropout \\
\hline 1 & 2D Convolutional Layer & - & 512 & 3 & ReLU & No \\
\hline 2 & 2D Convolutional Layer & - & 512 & 3 & ReLU \\
\hline 3 & 2D Convolutional Layer & - & 512 & 3 & ReLU \\
\hline 4 & 2D Convolutional Layer & - & 512 & 1 & No \\
\hline 5 & Dense Layer & 256 & - & - & ReLU & ReLU \\
\hline 6 & Dense Layer & 128 & - & - & Yes \\
\hline 7 & Dense Layer & 68 & - & - & Sigmoid & Yes \\
\hline
\end{tabular}

Table 4.2: MLP layer's hyperparameters.

\begin{tabular}{|c|c|c|c|c|c|c|}
\hline Layer Number & Type & Number of Neurons & Number of Filters & Filter Size & Activation Function & Dropout \\
\hline 1 & Dense Layer & 136 & - & - & ReLU & No \\
\hline 2 & Dense Layer & 68 & - & - & ReLU & No \\
\hline 3 & Dense Layer & 1 & - & - & Linear & No \\
\hline
\end{tabular}

into account the order of magnitude of the errors. The number of backpropagation iterations was 
1000 and 5000. Regarding initial candidate keypoints and adapted candidate keypoints, the average MSE, its standard deviation and the maximum and minimum errors were obtained. Table 4.3, Table 4.4 and Table 4.5 show results for the three possible initial candidate keypoints status: candidate keypoints were the result of the prediction obtained with the model described in [6] in images of the test set; candidate keypoints were the result of random generated values; candidate keypoints were coordinates with value 0. Figure 4.10, Figure 4.11 and Figure 4.12 show the dif-

Table 4.3: Results for the backpropagation applied to the candidate keypoints which were the result of the prediction obtained with the model described in [6] in images of the test set. Best results are highlighted in bold.

\begin{tabular}{|l|c|c|c|c|c|}
\hline Real MSE & Mean & Standard Deviation & Max & Min & Number of Steps \\
\hline Ground-Truth \& Initial Candidates & $\mathbf{0 . 0 0 1 1 6}$ & $\mathbf{0 . 0 0 1 5 0}$ & 0.00659 & $\mathbf{0 . 0 0 0 1 3}$ & - \\
\hline Ground-Truth \& Adapted Candidates & 0.00118 & 0.00151 & $\mathbf{0 . 0 0 6 5 1}$ & 0.00014 & $\mathbf{1 0 0 0}$ \\
\hline Ground-Truth \& Adapted Candidates & 0.00139 & 0.00158 & 0.00656 & 0.00017 & $\mathbf{5 0 0 0}$ \\
\hline
\end{tabular}

Table 4.4: Results for the backpropagation applied to the candidate keypoints which were the result of random generated values. Best results are highlighted in bold.

\begin{tabular}{|l|c|c|c|c|c|}
\hline Real MSE & Mean & Standard Deviation & Max & Min & Number of Steps \\
\hline Ground-Truth \& Initial Candidates & 0.11286 & 0.01700 & 0.15130 & 0.07088 & - \\
\hline Ground-Truth \& Adapted Candidates & 0.11087 & 0.01639 & 0.14704 & 0.07051 & $\mathbf{1 0 0 0}$ \\
\hline Ground-Truth \& Adapted Candidates & $\mathbf{0 . 1 0 5 1 9}$ & $\mathbf{0 . 0 1 4 9 2}$ & $\mathbf{0 . 1 4 0 6 3}$ & $\mathbf{0 . 0 6 9 5 4}$ & $\mathbf{5 0 0 0}$ \\
\hline
\end{tabular}

Table 4.5: Results for the backpropagation applied to the candidate keypoints which were coordinates with value 0 . Best results are highlighted in bold.

\begin{tabular}{|l|c|c|c|c|c|}
\hline Real MSE & Mean & Standard Deviation & Max & Min & Number of Steps \\
\hline Ground-Truth \& Initial Candidates & 0.27012 & 0.03186 & 0.34395 & 0.21046 & - \\
\hline Ground-Truth \& Adapted Candidates & 0.25837 & 0.03128 & 0.33107 & 0.20054 & $\mathbf{1 0 0 0}$ \\
\hline Ground-Truth \& Adapted Candidates & $\mathbf{0 . 2 1 9 2 9}$ & $\mathbf{0 . 0 2 8 6 5}$ & $\mathbf{0 . 2 8 6 7 1}$ & $\mathbf{0 . 1 6 7 2 4}$ & $\mathbf{5 0 0 0}$ \\
\hline
\end{tabular}

ferences between the application of 1000 and 5000 backpropagation steps for the three possible initial candidate keypoints status: candidate keypoints were the result of the prediction obtained with the model described in [6] in images of the test set; candidate keypoints were the result of random generated values; candidate keypoints were coordinates with value 0 . Please note that, although some differences may seem unnoticed, they have high impact on the final MSE. Also, since the order of magnitude of the quality/error values predicted by the network is small, one had to deal with a high number of steps for a small learning rate.

\subsubsection{Deep Iterative Refinement of Keypoint Detection}

This model was implemented in PyTorch [135]. The data preparation process was the same as in previous Subsection. Regarding training phase, the model was trained during 300 epochs, with Adadelta [136] as optimization function, and the model with lower loss on the validation set was 

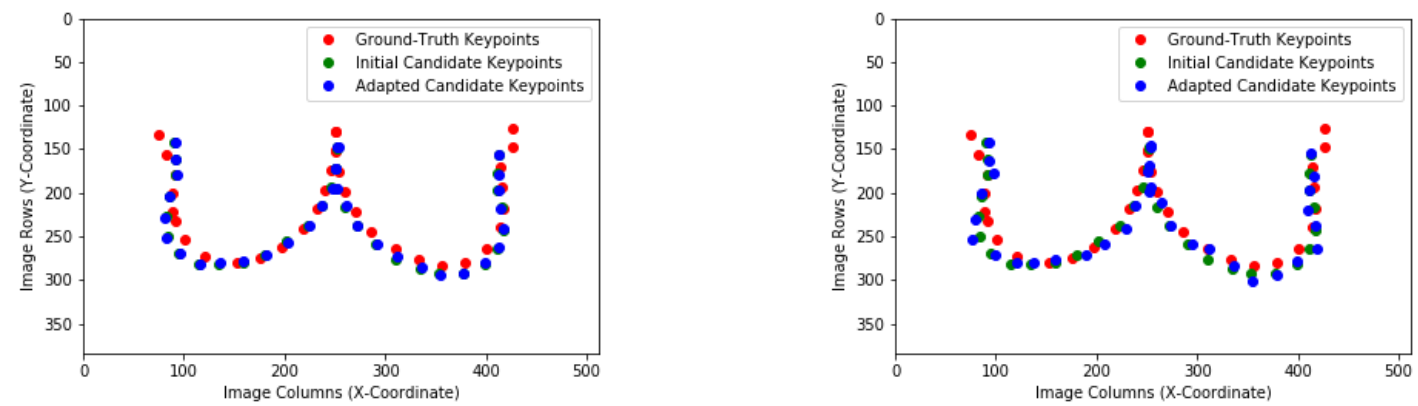

Figure 4.10: Differences between the application of 1000 and 5000 backpropagation steps to the candidate keypoints which were the result of prediction of the model described in [6] in images of the test set.
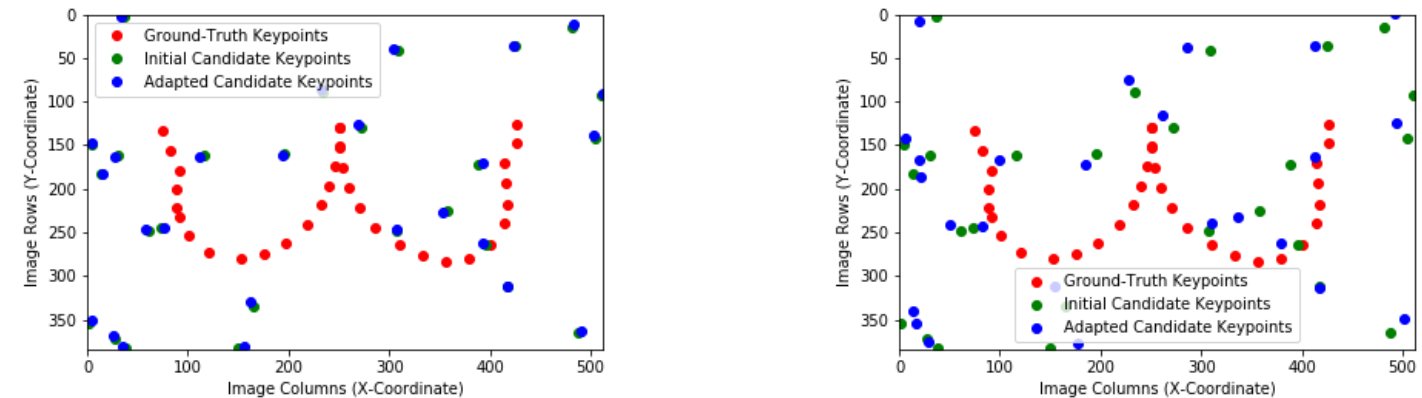

Figure 4.11: Differences between the application of 1000 and 5000 backpropagation steps to the candidate keypoints which were the result of random generated values.
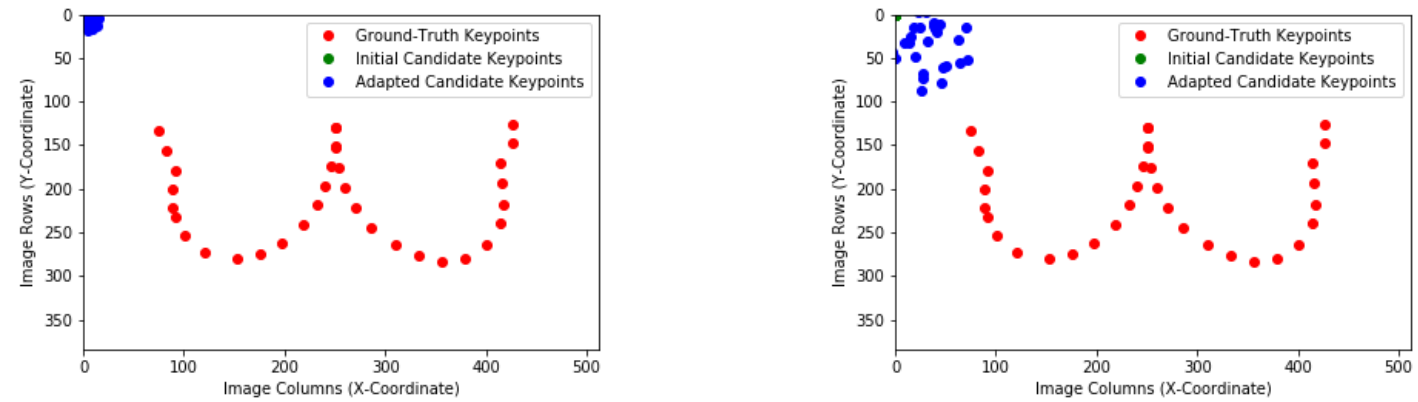

Figure 4.12: Differences between the application of 1000 and 5000 backpropagation steps to the candidate keypoints which were coordinates with value 0 .

saved. For this approach, a different strategy for the loss function was designed:

$$
\mathscr{L}_{\text {model }}=\lambda_{1} \mathscr{L}_{\text {quality }}+\lambda_{2} \mathscr{L}_{\text {refined keypoints }}
$$

where $\lambda_{1}$ and $\lambda_{2}$ represent non-negative weights attributed to each loss. The loss of the quality (regularization term) is computed the same way as presented in Equation 4.8. The loss of the refined keypoints (main goal) is calculated as the MSE between the predicted refined keypoints, $\hat{k}$ 
and the ground-truth keypoints, $k$ :

$$
\mathscr{L}_{\text {refined keypoints }}=\operatorname{MSE}(\hat{k}, k)
$$

Table 4.6, Table 4.7 and Table 4.8 present the hyperparameters for the Intermediate MLP, the Quality MLP and Keypoints MLP, respectively. Table 4.9, Table 4.10 and Table 4.11 show results

Table 4.6: Intermediate MLP layer's hyperparameters.

\begin{tabular}{|c|c|c|c|c|c|c|}
\hline Layer Number & Type & Number of Neurons & Number of Filters & Filter Size & Activation Function & Dropout \\
\hline 1 & Dense Layer & 68 & - & - & ReLU & No \\
\hline 2 & Dense Layer & 34 & - & - & ReLU & No \\
\hline 3 & Dense Layer & 22 & - & - & Linear & No \\
\hline
\end{tabular}

Table 4.7: Quality MLP layer's hyperparameters.

\begin{tabular}{|c|c|c|c|c|c|c|}
\hline Layer Number & Type & Number of Neurons & Number of Filters & Filter Size & Activation Function & Dropout \\
\hline 1 & Dense Layer & 11 & - & - & ReLU & No \\
\hline 2 & Dense Layer & 6 & - & - & ReLU & No \\
\hline 3 & Dense Layer & 1 & - & - & Linear & No \\
\hline
\end{tabular}

Table 4.8: Keypoints MLP layer’s hyperparameters.

\begin{tabular}{|c|c|c|c|c|c|c|}
\hline Layer Number & Type & Number of Neurons & Number of Filters & Filter Size & Activation Function & Dropout \\
\hline 1 & Dense Layer & 44 & - & - & ReLU & No \\
\hline 2 & Dense Layer & 58 & - & - & ReLU & No \\
\hline 3 & Dense Layer & 68 & - & - & Linear & No \\
\hline
\end{tabular}

for the three possible initial candidate keypoints status: candidate keypoints were the result of the prediction obtained with the model described in [6] in images of the test set; candidate keypoints were the result of random generated values; candidate keypoints were coordinates with value 0 . Figure 4.13, Figure 4.14 and Figure 4.15 show examples of the evolution of candidate keypoint coordinates from the three possible initial states.

Table 4.9: Results for the iterative refinement model applied to the candidate keypoints which were the result of the prediction obtained with the model described in [6] in images of the test set.

\begin{tabular}{|l|c|c|c|c|}
\hline Real MSE & Mean & Standard Deviation & Max & Min \\
\hline Ground-Truth \& Initial Candidates & $\mathbf{0 . 0 0 1 1 6}$ & $\mathbf{0 . 0 0 1 5 0}$ & $\mathbf{0 . 0 0 6 5 9}$ & $\mathbf{0 . 0 0 0 1 3}$ \\
\hline Ground-Truth \& Adapted Candidates & 0.00218 & 0.00164 & 0.00750 & 0.00028 \\
\hline
\end{tabular}

Table 4.10: Results for the iterative refinement model applied to the candidate keypoints which were the result of random generated values.

\begin{tabular}{|l|c|c|c|c|}
\hline Real MSE & Mean & Standard Deviation & Max & Min \\
\hline Ground-Truth \& Initial Candidates & 0.11286 & 0.01700 & 0.15130 & 0.07088 \\
\hline Ground-Truth \& Adapted Candidates & $\mathbf{0 . 0 0 2 3 3}$ & $\mathbf{0 . 0 0 1 5 0}$ & $\mathbf{0 . 0 0 6 3 3}$ & $\mathbf{0 . 0 0 0 3 3}$ \\
\hline
\end{tabular}


Table 4.11: Results for the iterative refinement model applied to the candidate keypoints which were coordinates with value 0 .

\begin{tabular}{|l|c|c|c|c|}
\hline Real MSE & Mean & Standard Deviation & Max & Min \\
\hline Ground-Truth \& Initial Candidates & 0.27012 & 0.03186 & 0.34395 & 0.21046 \\
\hline Ground-Truth \& Adapted Candidates & $\mathbf{0 . 0 0 3 4 5}$ & $\mathbf{0 . 0 0 2 9 4}$ & $\mathbf{0 . 0 1 2 9 3}$ & $\mathbf{0 . 0 0 0 4 5}$ \\
\hline
\end{tabular}

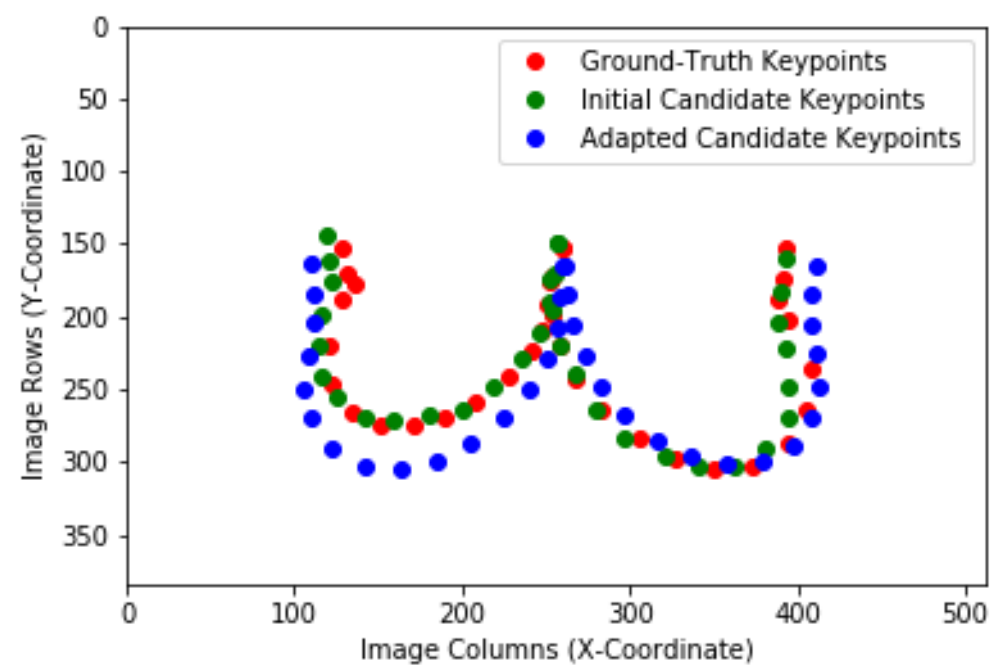

Figure 4.13: Evolution of candidate keypoint coordinates (predictions from [6]), from the initial to the adapted states, against the ground-truth.

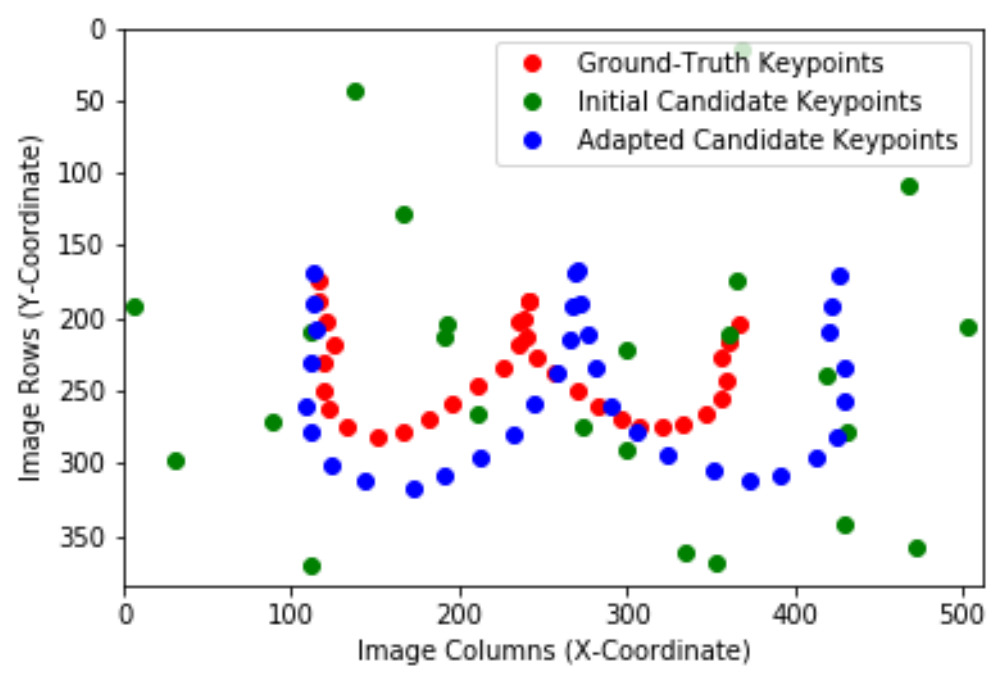

Figure 4.14: Evolution of candidate keypoint coordinates (random generated values), from the initial to the adapted states, against the ground-truth. 


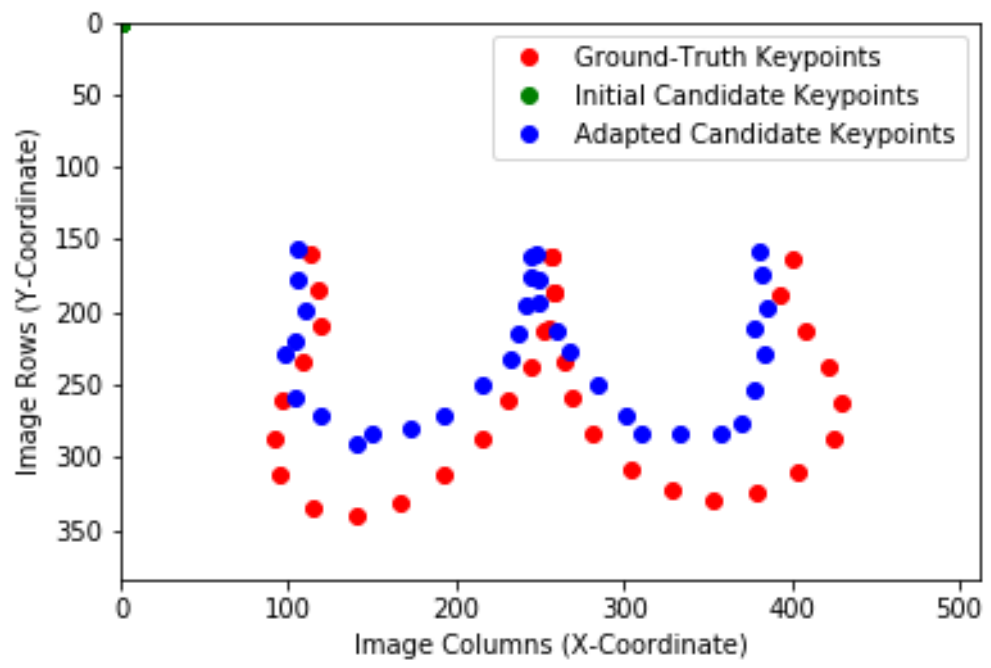

Figure 4.15: Evolution of candidate keypoint coordinates (coordinates with value 0), from the initial to the adapted states, against the ground-truth.

\subsection{Conclusions}

Both proposed models were functional, however, they were not able to improve the state of the art results, i.e., the ones published by Silva et al. in [6]. Regarding the first approach, explained in Subsection 4.2.1, there are some considerations that have to be taken into account: inference of quality has always an associated error amplitude, which means, that the inference will always have some deviations from the real quality value; this will have implications when performing backpropagation in the candidate keypoints, due to the fact, that, in each step, the inferred quality value will always diminish, but that does not necessarily mean that the real quality value (i.e., the MSE between candidate keypoints and ground-truth keypoints) has diminished. Also, although results are presented for different values of backpropagation steps, an optimal value still needs to be found; this is crucial, since, following the main conclusions from Fernandes et al. in [11], too much backpropagation steps may lead to a deterioration of the adapted candidate keypoints. On behalf of the second approach, the refined keypoints predictions have better performances, but still need further improvements, regarding the fact that they did not improve the results obtained with the model proposed by Silva et al. in [6]. It is important to notice that, even with data augmentation, the dataset used to train the models may not be large enough to address these specific tasks; the applied data augmentation parameters may also not be enough, and, it is possible that the models need an even higher variety of examples to properly learn. It is also important to mention that this was the first work which used a quality-driven approach for a regression task; this means that there is space for improvements. Also, it is still not clear that this approach will lead to success, considering this kind of tasks. For all these reasons, these models still need 
further research and development in order to be considered viable options to solve this thesis' main problem. 


\section{Chapter 5}

\section{A Web Application for Automatic Keypoint Detection}

\section{Contents}

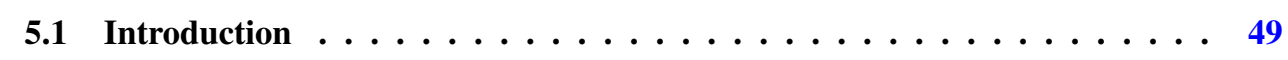

5.2 Use-Cases and Proposed Architecture $\ldots \ldots \ldots \ldots \ldots \ldots \ldots$

5.3 Implementation and Prototype . . . . . . . . . . . 51

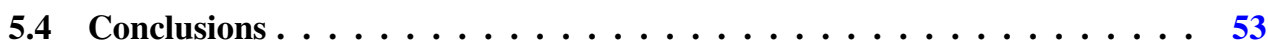

\subsection{Introduction}

As stated in Chapter 1, one of the expected outcomes of this project is the development of a web application to be used for keypoint detection and aesthetic assessment. The main objective is to substitute BCCT.core [5], which is only available offline, by an application that could be easily accessed through a web browser by a healthcare professional.

\subsubsection{Motivation}

Currently, in order to use BCCT.core [5], users must download, install and set-up the software on their computers; these three steps may be considered a burden for some of the users and they also imply that the software will use their computational resources to properly function. On the other hand, fundamental services such as software maintenance (updates and/or upgrades) or support could be difficult in an offline setting. To overcome these issues, and taking into account that most of the healthcare institutions have an Internet connection [140], it was decided that the best option would be to move BCCT.core into a web-based application. This way, users would only need to deal with a simple website registration in order to use all the functionalities of the software; the rest would be handled in the background, on the server where the application is hosted. Moreover, the field of web development is in a trending evolution, being able to keep up with most of the 
market's new requirements [141]. The following Sections describe the use-cases and the proposed architecture for such an application.

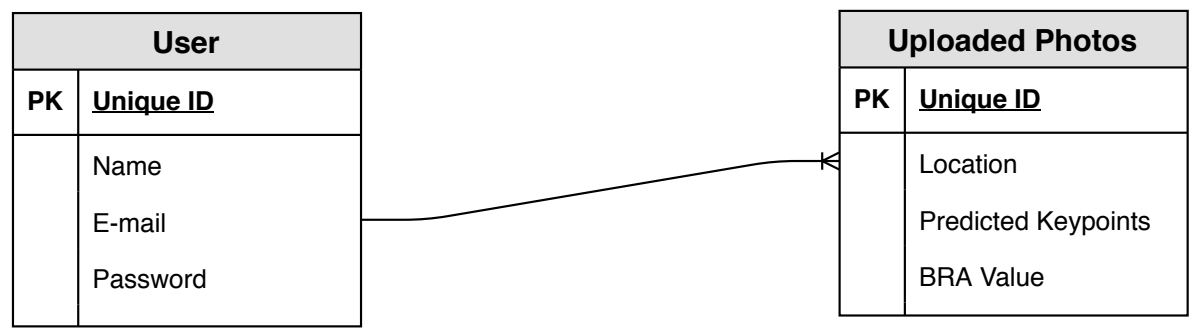

Figure 5.1: Proposed Entity-Relationship model of the application's database.

\subsection{Use-Cases and Proposed Architecture}

The starting point for the establishment of use-cases for this web-based application for automatic keypoint detection was BCCT.core [5]; the main functionalities will not require major improvements, however, the transition from offline to online setup has a big impact on the new practicalities of the application, and this should be taken into account on the redesign of the software. The identified use-cases, from the user side, were:

1. The user should log in to enter the application: with the application in an online setting, it is crucial that measures that take into account the privacy of the clinical data are implemented, and, as such, it is believed that a login system for user authentication inside the application is of utmost importance.

2. The user may upload medical photos: to perform the keypoint detection, the application should have an intuitive interface so users can upload the photos that are going to be subjected to evaluation.

3. The user may consult and change the detection records: it is important that the user has access to the evaluated photos and to the predicted keypoints and he/she should be able to manually correct the model's predictions.

4. The user may erase all the records from the system: once, again, since one is dealing with sensitive clinical data, it is important that the users have the possibility to reset their records.

From the server side, the system should have a database. This database will contain:

1. User's information: name, e-mail and password.

2. Uploaded Photo's information: location, predicted keypoints, the associated user and BCCT assessment information (in this case, it is used BRA value as an example). 
Regarding the type of relationship, it can be seen that it is a one-to-many relationship, i.e., an user can upload many photos. Figure 5.1 shows the proposed Entity-Relationship model of the application's database. Another fundamental requirement is a back-end capable of interacting with deep learning models created in Python; the server should have enough space to host such models. Figure 5.5 shows the proposed work-flow for the application, with all the interactions between front-end and back-end.

\subsection{Implementation and Prototype}

The web application prototype was developed with Django ${ }^{1}$, a free and open source high-level Python web framework that promotes fast development, security and scalability. The main reason behind this choice is indeed related with the fact that the deep learning models are written in Python, and since Django is also powered by the same programming language, this applicationmodels interaction would be simpler. Moreover, Django permits the creation and manipulation of databases through Python, simplifying, once again, all the development that requires interaction between the front-end and the back-end. With everything on the back-end being handled by Django, one has to deal with the front-end. The front-end of an application is directly related to its usability and user experience. In terms of development, it was used HTML5, CSS and JavaScript, which are the standard code languages for web development.

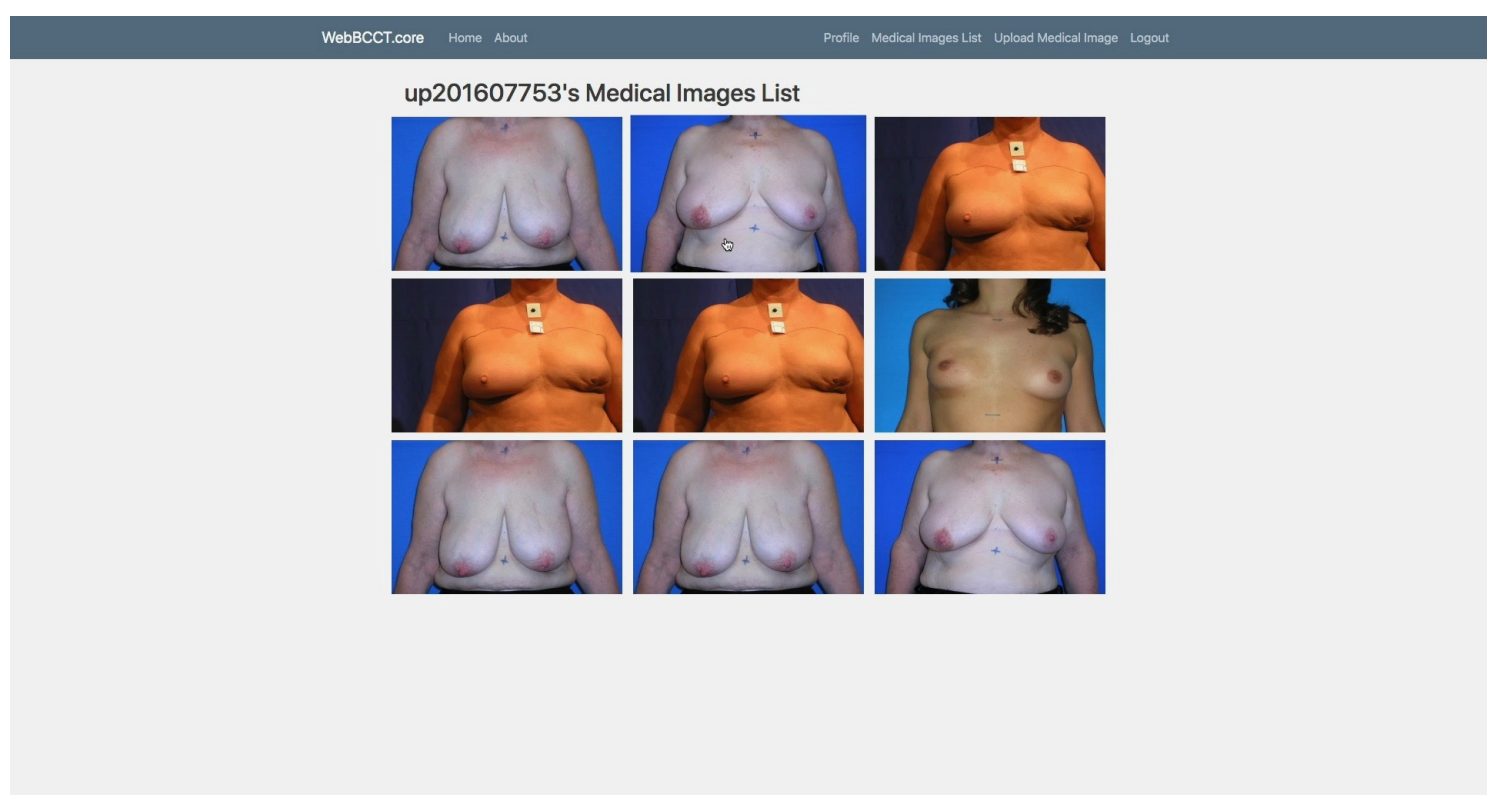

Figure 5.2: Web application's main interface.

One of the main objectives is to have an interface that allows users to perform their tasks (i.e., keypoint detection and/or aesthetic assessment) as simple as possible; to do that, it was

\footnotetext{
${ }^{1}$ More information at https://www.djangoproject.com/
} 


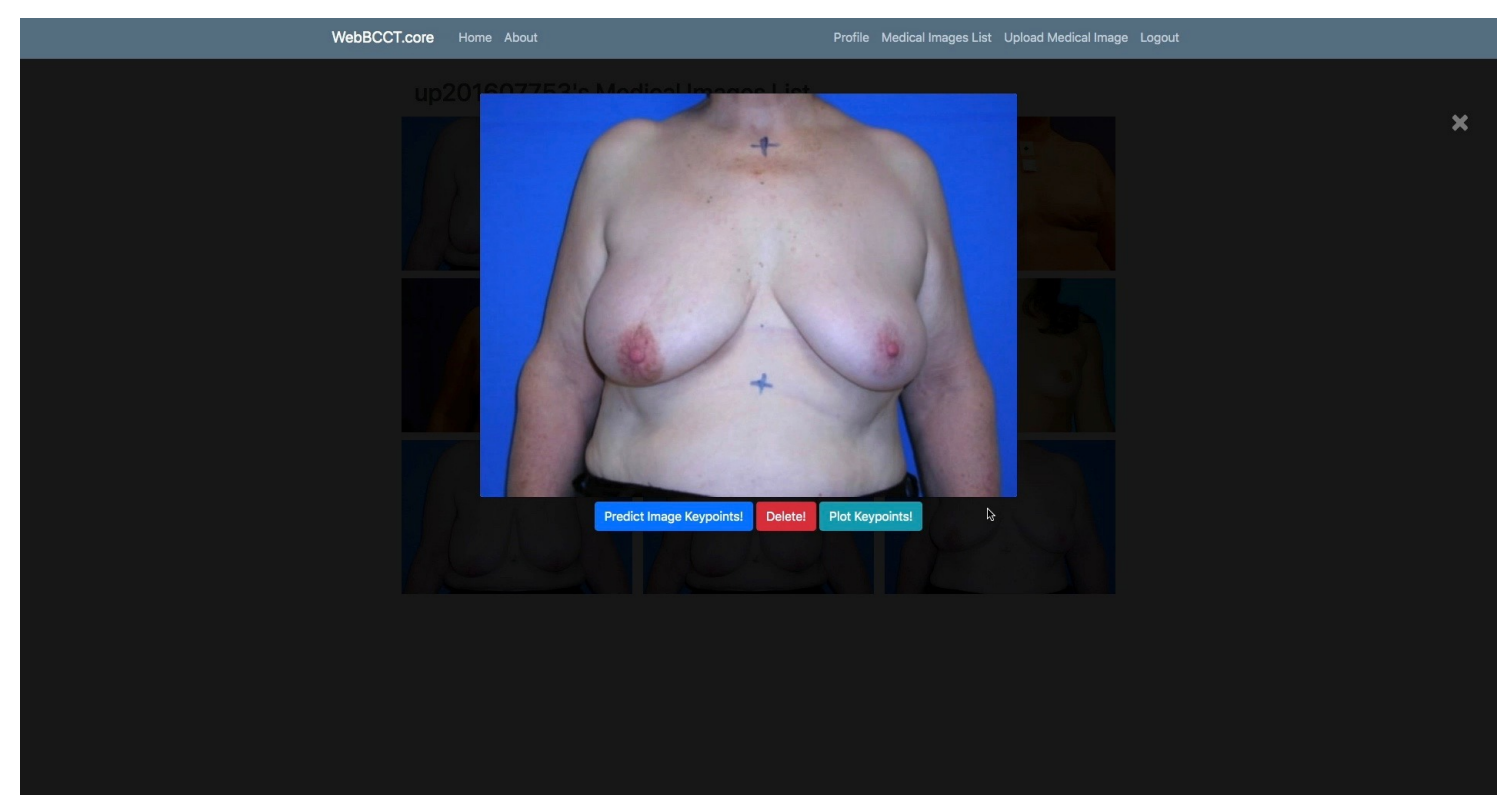

Figure 5.3: Web application's images' functions interface.

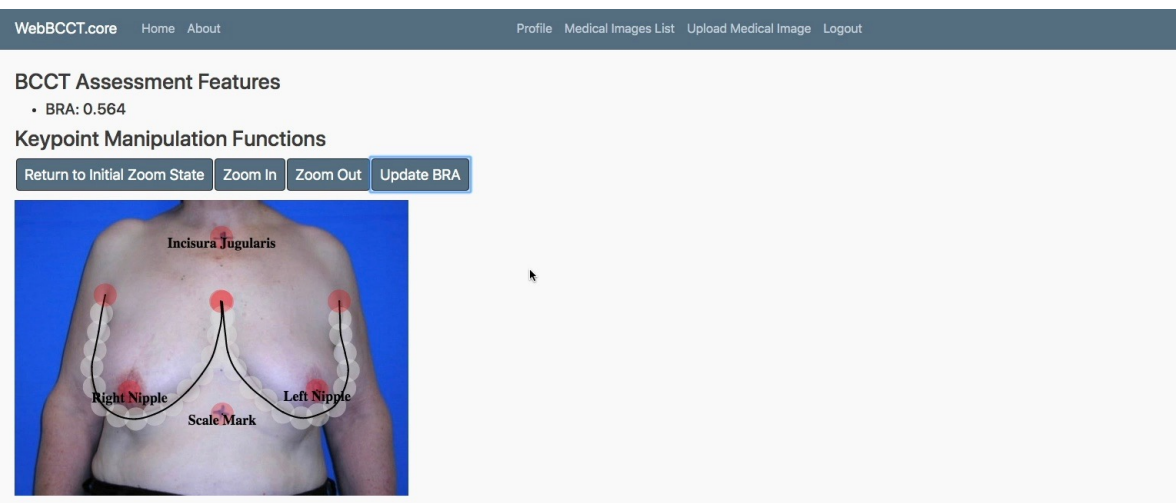

Figure 5.4: Web application's plot keypoints interface.

decided to incorporate all the functionalities as buttons, where the user clicks and sees the results, without the need to check what is happening in the background. An administration interface was also developed, since, in reality, such a system requires a professional who can properly manage, update and maintain it, while giving some kind of support to the users. Figure 5.2 shows the application interface; the user is logged and is already on the page where it is possible to view the uploaded medical images. Figure 5.3 shows the interface that allows users to call several functions 
with the help of buttons. Figure 5.4 shows the interface where the user can see and manipulate the predicted keypoints and save the BRA value into the database.

\subsection{Conclusions}

It was possible to achieve a fully working prototype, capable of fulfilling all the previously stated use-cases and representing, thus, a reliable proof of concept that can act as a starting point for the final deployment of a final version of the web application for automatic keypoint detection and BCCT assessment. Regarding the fact that the system is in development mode, the next steps would be, for example:

- Change the database framework (currently the system uses SQLite ${ }^{2}$ ) to a scalable one (for example, PostgreSQL ${ }^{3}$ ), which is supported by Django. Important to remind that the method of interacting with the database stays almost untouched; once again, Django handles all the background work.

- Add the computation of more BCCT assessment features, besides BRA, and the computation of the BCCT assessment itself (i.e., automatically classify images according Harris scale [64]).

\footnotetext{
${ }^{2}$ More information at https://sqlite.org/index.html

${ }^{3}$ More information at https://www.postgresql.org/
} 


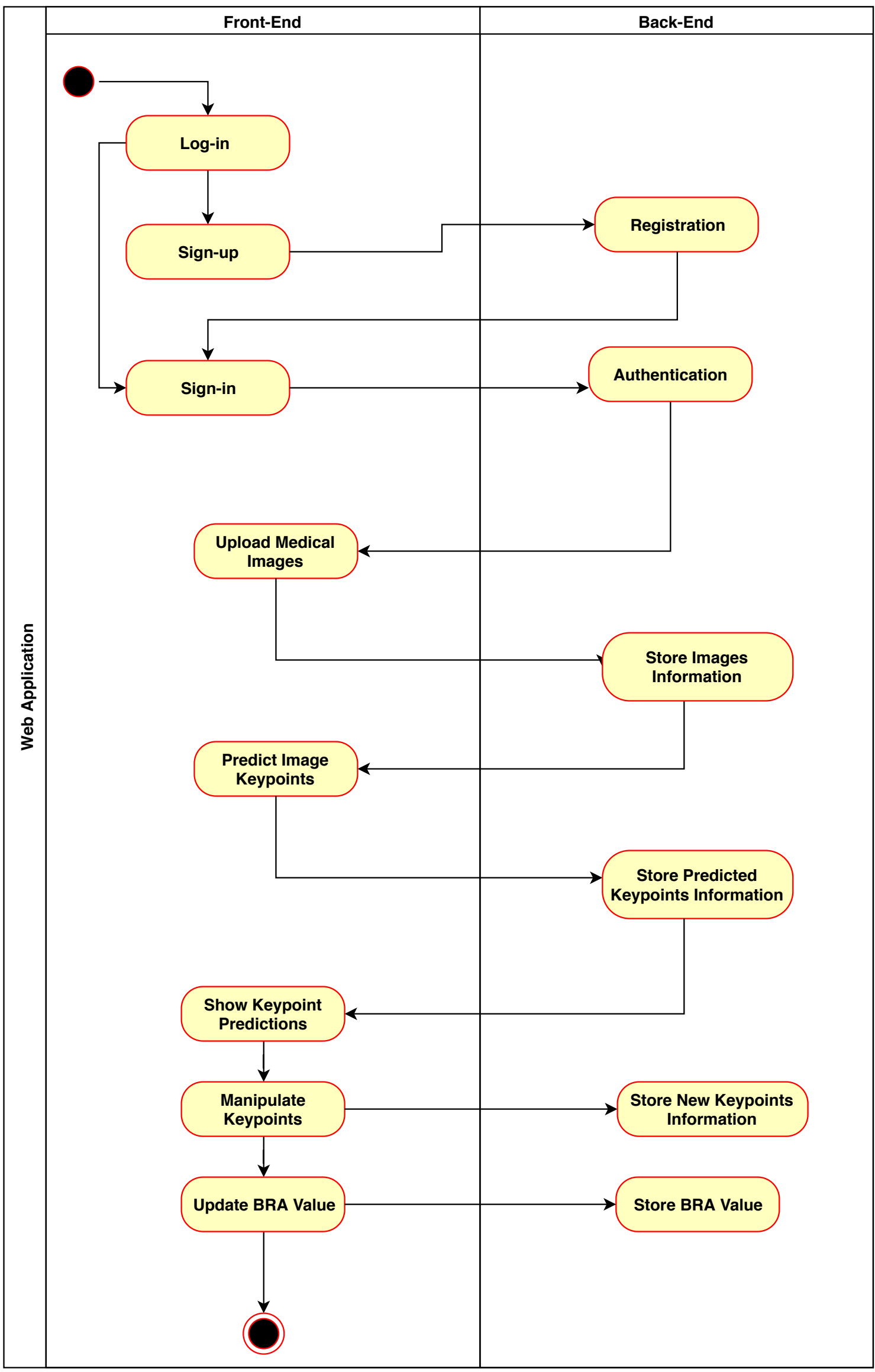

Figure 5.5: Proposed work-flow for the application. 


\section{Chapter 6}

\section{Conclusions and Future Work}

\section{Contents}

6.1 Overview ...................... 55

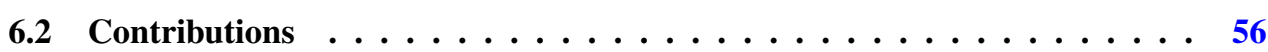

6.3 Future Work $\ldots \ldots \ldots \ldots \ldots \ldots \ldots \ldots \ldots \ldots$

\subsection{Overview}

In this thesis, a literature review towards the importance of BCCT assessment and the computeraided aesthetic classification of BCCT outcomes was done. The state of the art methodology for keypoint detection, proposed by Silva et al. [6] was analysed and considered as the reference work for comparison with other different approaches. Then, the initially defined goals in Chapter 1 were addressed:

1. The investigation of different deep learning approaches for fiducial point detection.

Two different approaches were explored: image segmentation for breast contour detection and quality-driven keypoint detection. In the first one, reference works $[7 ; 131 ; 9 ; 10]$ on image segmentation were described and used in the dataset described in Chapter 2. GCN and U-Net achieved near state of the art results, however, these do not provide enough stability to conclude that such a method can be used to properly detect breast contour. In the second approach, an overview of the works developed by Fernandes et al. [11] and Rebelo et al. [12] was made and, based on this research, two novel quality driven methodologies for keypoint detection were presented and discussed: one which is based on a network that is trained to learn how to predict the quality of a given image and candidate keypoints pair, and the application of backpropagation to improve the candidate keypoints; and, another which is based on the use of quality inference during training, as a regularization term, and the use of the keypoints prediction output as an input for the next training iteration, to iteratively refine the keypoints prediction. Both models are functional, however, results show that, for now, they are not able to surpass nor improve the state of the art [6]. 
2. The development of a web application with the best-researched technique as an online service, based on HTML5, CSS, JavaScript and PHP.

A working prototype was developed in Django; it has both server and client sides. From the server side, the application has a database, where user and images' information are stored, and contains all the needed mechanisms to execute and handle all the deep learning models, which are written in Python. On the other hand, the client side is responsible for all the user interfaces and all the buttons that permit an user-friendly and intuitive interaction with the application.

\subsection{Contributions}

The contributions of this thesis are the following:

- Evaluation of different image segmentation DNNs performances and their possible usage for breast contour detection.

- Implementation of a keypoint quality prediction network, based on the MSE between candidate keypoints and ground-truth keypoints.

- Application of the backpropagation algorithm to improve the quality of the candidate keypoints, by gradient descent.

- Implementation of an iterative keypoint refinement network, which uses the quality concept as a regularization method for keypoint prediction and which uses the output of the network as input in each training iteration.

- Development of a web application, written in Django, HTML, CSS and JavaScript which integrates the deep learning model, in Python, and allows the user to perform keypoint prediction directly from a button.

\subsection{Future Work}

\subsubsection{DeepLabV3+ for Breast Image Segmentation}

Until now, DeepLabV3+ [10] is the state of the art network in image segmentation. Although it was not used in this thesis due to the lack of computational resources, it would be important and interesting to check if it is capable of achieving better and stable results, which could permit the breast contour detection from predicted segmentation masks.

\subsubsection{Recurrent Neural Networks for Keypoint Prediction}

It is possible to address the task of keypoint prediction from a different perspective. One may consider that the array of keypoints coordinates are ordered sequences, i.e., each coordinate value 
depends on its position in the sequence. For this situation, the use of recurrent neural networks (RNN), which are widely used in time-series, machine translation, speech recognition or sentiment analysis, is advised. On the other hand, the main goal of the project, from which this thesis is part, is to start from an input image and then predict its keypoints; based on this condition, the training of such a neural network would require the integration of RNNs and CNNs. The work published by Wang et al. [142], gives some insights on this topic.

\subsubsection{Graph Neural Networks for Keypoint Prediction}

Already stated by Cardoso et al. [82], keypoint prediction can also be thought as a graph problem. To address this problem, one would have to model each image as a graph and then train a graph neural network (GNN) on finding the shortest path between two nodes, which, in this case, would be the endpoints. Once again, the main objective is to start from an input image, so one could first use the model from Silva et al. to predict the endpoints, then model the image as graph (as in [82]), and start from the predicted endpoints to train the GNN, on finding its shortest path, which, by principle, should correspond to the breast contour. There is already published work on how to train a GNN to learn the shortest path between two graph nodes, by DeepMind [143].

\subsubsection{Different Metrics for Quality Evaluation}

In the scope of this thesis, the metric that was used to evaluate quality was MSE. There is not, however, evidence that this is the most appropriate metric to do so. Some preliminary tests have been done with the mean absolute error (MAE) and the error with respect to the spline obtained with the contour keypoints, used in [6], but further work is required. The use of a different metric (e.g., the error with respect to the spline obtained with the contour keypoints) could make the training richer since it could lead the network to learn new features that could improve its performance.

\subsubsection{Data Acquisition to Improve Training Performance}

One of the problems involved in the difficulty of training the models proposed in this thesis is the size of the currently available dataset, which only contains 221 images with the correspondent keypoints. A higher number of training images and keypoints could benefit the training of DNNs applied to this task.

\subsubsection{Deployment of the Web Application for Healthcare Professionals}

The prototype presented in this thesis is a proof of concept with a specifically designed architecture, however, as already stated, it is fully scalable and deployable. At the moment, users can work on keypoints prediction, using an image as input. What is aimed, in fact, is an application that uses this information to predict the aesthetic classification; this functionality needs to be integrated. Moreover, during the prototype development, no interviews with healthcare professionals 
to acquire more use cases nor usability tests were done; this is required to have a final application that fits user needs and, at the same time, that is intuitive for them. 


\section{References}

[1] WebMD. A Visual Guide to Breast Cancer.

https://www.webmd.com/breast-cancer/ss/slideshow-breast-cancer-overview, Last accessed on 2019-06-23.

[2] Cancer Research UK. Breast cancer, October 2017.

https://www.cancerresearchuk.org/about-cancer/breast-cancer/treatment/surgery/remove-

just-area-cancer, Last accessed on 2019-06-23.

[3] Cancer Research UK. Breast cancer, October 2017.

https://www.cancerresearchuk.org/about-cancer/breast-cancer/treatment/surgery/removewhole-breast, Last accessed on 2019-06-23.

[4] Wilfried Krois, Alexander Ken Romar, Thomas Wild, Peter Dubsky, Ruth Exner, Peter Panhofer, Raimund Jakesz, Michael Gnant, and Florian Fitzal. Objective breast symmetry analysis with the breast analyzing tool (BAT): improved tool for clinical trials. Breast Cancer Research and Treatment, 164(2):421-427, July 2017.

[5] Jaime S. Cardoso and Maria J. Cardoso. Towards an intelligent medical system for the aesthetic evaluation of breast cancer conservative treatment. Artificial Intelligence in Medicine, 40(2):115-126, June 2007.

[6] Wilson Silva, Eduardo Castro, Maria J. Cardoso, Florian Fitzal, and Jaime S. Cardoso. Deep Keypoint Detection for the Aesthetic Evaluation of Breast Cancer Surgery Outcomes. In Proceedings of the IEEE International Symposium on Biomedical Imaging (ISBI'19), 2019.

[7] Evan Shelhamer, Jonathan Long, and Trevor Darrell. Fully Convolutional Networks for Semantic Segmentation. arXiv:1605.06211 [cs], May 2016. arXiv: 1605.06211.

[8] Olaf Ronneberger, Philipp Fischer, and Thomas Brox. U-Net: Convolutional Networks for Biomedical Image Segmentation. arXiv:1505.04597 [cs], May 2015. arXiv: 1505.04597.

[9] Chao Peng, Xiangyu Zhang, Gang Yu, Guiming Luo, and Jian Sun. Large Kernel Matters - Improve Semantic Segmentation by Global Convolutional Network. arXiv:1703.02719 [cs], March 2017. arXiv: 1703.02719.

[10] Liang-Chieh Chen, Yukun Zhu, George Papandreou, Florian Schroff, and Hartwig Adam. Encoder-Decoder with Atrous Separable Convolution for Semantic Image Segmentation. arXiv:1802.02611 [cs], February 2018. arXiv: 1802.02611.

[11] Kelwin Fernandes, Ricardo Cruz, and Jaime S. Cardoso. Deep Image Segmentation by Quality Inference. In 2018 International Joint Conference on Neural Networks (IJCNN), pages 1-8, Rio de Janeiro, July 2018. IEEE. 
[12] José Rebelo, Kelwin Fernandes, and Jaime S Cardoso. Quality-based Regularization for Iterative Deep Image Segmentation. page 4, 2019.

[13] Maria J. Cardoso, Jaime Cardoso, Ana C. Santos, Conny Vrieling, David Christie, Göran Liljegren, Isabel Azevedo, Jørgen Johansen, José Rosa, Natália Amaral, Rauni Saaristo, Virgilio Sacchini, Henrique Barros, and Manuel C. Oliveira. Factors Determining Esthetic Outcome after Breast Cancer Conservative Treatment. The Breast Journal, 13(2):140-146, March 2007.

[14] Williams Street. Cancer Facts \& Figures 2018. page 76, 2018.

[15] Rebecca L. Siegel, Kimberly D. Miller, and Ahmedin Jemal. Cancer statistics, 2018. CA: A Cancer Journal for Clinicians, 68(1):7-30, January 2018.

[16] Ahmedin Jemal, Freddie Bray, Melissa M. Center, Jacques Ferlay, Elizabeth Ward, and David Forman. Global cancer statistics. CA: A Cancer Journal for Clinicians, 61(2):6990, March 2011.

[17] Colin Mathers, Doris Ma Fat, J. T. Boerma, and World Health Organization, editors. The global burden of disease: 2004 update. World Health Organization, Geneva, Switzerland, 2008. OCLC: ocn264018380.

[18] Bernard W Stewart, Chris Wild, International Agency for Research on Cancer, and World Health Organization. World cancer report 2014. 2014. OCLC: 1013966433.

[19] Freddie Bray, Jacques Ferlay, Isabelle Soerjomataram, Rebecca L. Siegel, Lindsey A. Torre, and Ahmedin Jemal. Global cancer statistics 2018: GLOBOCAN estimates of incidence and mortality worldwide for 36 cancers in 185 countries. CA: A Cancer Journal for Clinicians, $0(0)$.

[20] Helder P. Oliveira, Jaime S. Cardoso, Andre Magalhaes, and Maria J. Cardoso. Methods for the Aesthetic Evaluation of Breast Cancer Conservation Treatment: A Technological Review. Current Medical Imaging Reviews, 9(1):32-46, April 2013.

[21] Connie Henke Yarbro, Debra Wujcik, and Barbara Holmes Gobel, editors. Cancer nursing: principles and practice. Jones and Bartlett Publishers, Sudbury, Mass, 7th ed edition, 2011. OCLC: ocn421361446.

[22] Sharon H Giordano, Aman U Buzdar, and Gabriel N Hortobagyi. Breast Cancer in Men. page 11 .

[23] Susan Ely and Anna N. Vioral. Breast Cancer Overview:. Plastic Surgical Nursing, 27(3):128-133, July 2007.

[24] Vincent T. DeVita, Theodore S. Lawrence, and Steven A. Rosenberg. DeVita, Hellman, and Rosenberg's cancer: Principles \& practice of oncology: Tenth edition. Wolters Kluwer Health Adis (ESP), 2015.

[25] Williams Street. Breast Cancer Facts \& Figures 2017-2018. page 44, 2017.

[26] Kevin C. Oeffinger, Elizabeth T. H. Fontham, Ruth Etzioni, Abbe Herzig, James S. Michaelson, Ya-Chen Tina Shih, Louise C. Walter, Timothy R. Church, Christopher R. Flowers, Samuel J. LaMonte, Andrew M. D. Wolf, Carol DeSantis, Joannie Lortet-Tieulent, Kimberly Andrews, Deana Manassaram-Baptiste, Debbie Saslow, Robert A. Smith, Otis W. 
Brawley, and Richard Wender. Breast Cancer Screening for Women at Average Risk: 2015 Guideline Update From the American Cancer Society. JAMA, 314(15):1599, October 2015.

[27] Robert A. Smith, Debbie Saslow, Kimberly Andrews Sawyer, Wylie Burke, Mary E. Costanza, W. Phil Evans, Roger S. Foster, Edward Hendrick, Harmon J. Eyre, and Steven Sener. American Cancer Society Guidelines for Breast Cancer Screening: Update 2003. CA: A Cancer Journal for Clinicians, 53(3):141-169, May 2003.

[28] David B. Thomas, Dao Li Gao, Roberta M. Ray, Wen Wan Wang, Charlene J. Allison, Fan Liang Chen, Peggy Porter, Yong Wei Hu, Guan Lin Zhao, Lei Da Pan, Wenjin Li, Chunyuan Wu, Zakia Coriaty, Ilonka Evans, Ming Gang Lin, Helge Stalsberg, and Steven G. Self. Randomized trial of breast self-examination in Shanghai: final results. Journal of the National Cancer Institute, 94(19):1445-1457, October 2002.

[29] V. F. Semiglazov, V. M. Moiseenko, A. G. Manikhas, S. A. Protsenko, R. S. Kharikova, R. T. Popova, N. Sh Migmanova, A. A. Orlov, N. Iu Barash, O. A. Ivanova, and V. G. Ivanov. [Interim results of a prospective randomized study of self-examination for early detection of breast cancer (Russia/St.Petersburg/WHO)]. Voprosy Onkologii, 45(3):265-271, 1999.

[30] W H Goodson. Clinical breast examination. Western Journal of Medicine, 164(4):355-358, April 1996.

[31] Benjamin O. Anderson, Therese B. Bevers, and Robert W. Carlson. Clinical Breast Examination and Breast Cancer Screening Guideline. JAMA, 315(13):1403, April 2016.

[32] S. McDonald, D. Saslow, and M. H. Alciati. Performance and Reporting of Clinical Breast Examination: A Review of the Literature. CA: A Cancer Journal for Clinicians, 54(6):345361, November 2004.

[33] J. K. Bobo. Findings From 752081 Clinical Breast Examinations Reported to a National Screening Program From 1995 Through 1998. Journal of the National Cancer Institute, 92(12):971-976, June 2000.

[34] Etta D. Pisano, R. Edward Hendrick, Martin J. Yaffe, Janet K. Baum, Suddhasatta Acharyya, Jean B. Cormack, Lucy A. Hanna, Emily F. Conant, Laurie L. Fajardo, Lawrence W. Bassett, Carl J. D’Orsi, Roberta A. Jong, Murray Rebner, Anna N. A. Tosteson, and Constantine A. Gatsonis. Diagnostic Accuracy of Digital versus Film Mammography: Exploratory Analysis of Selected Population Subgroups in DMIST. Radiology, 246(2):376-383, February 2008.

[35] Fabiano H. Souza, Eliana M. Wendland, Maria I. Rosa, and Carisi A. Polanczyk. Is full-field digital mammography more accurate than screen-film mammography in overall population screening? A systematic review and meta-analysis. The Breast, 22(3):217-224, June 2013.

[36] Karla Kerlikowske. Comparative Effectiveness of Digital Versus Film-Screen Mammography in Community Practice in the United States: A Cohort Study. Annals of Internal Medicine, 155(8):493, October 2011.

[37] Joy Melnikow, Joshua J. Fenton, Evelyn P. Whitlock, Diana L. Miglioretti, Meghan S. Weyrich, Jamie H. Thompson, and Kunal Shah. Supplemental Screening for Breast Cancer in Women With Dense Breasts: A Systematic Review for the U.S. Preventive Services Task Force. Annals of Internal Medicine, 164(4):268-278, February 2016. 
[38] Alberto S. Tagliafico, Massimo Calabrese, Giovanna Mariscotti, Manuela Durando, Simona Tosto, Francesco Monetti, Sonia Airaldi, Bianca Bignotti, Jacopo Nori, Antonella Bagni, Alessio Signori, Maria Pia Sormani, and Nehmat Houssami. Adjunct Screening With Tomosynthesis or Ultrasound in Women With Mammography-Negative Dense Breasts: Interim Report of a Prospective Comparative Trial. Journal of Clinical Oncology: Official Journal of the American Society of Clinical Oncology, March 2016.

[39] Debbie Saslow, Carla Boetes, Wylie Burke, Steven Harms, Martin O. Leach, Constance D. Lehman, Elizabeth Morris, Etta Pisano, Mitchell Schnall, Stephen Sener, Robert A. Smith, Ellen Warner, Martin Yaffe, Kimberly S. Andrews, Christy A. Russell, and American Cancer Society Breast Cancer Advisory Group. American Cancer Society guidelines for breast screening with MRI as an adjunct to mammography. CA: a cancer journal for clinicians, 57(2):75-89, April 2007.

[40] A Grisotti. Immediate reconstruction after partial mastectomy. page 12, 1994.

[41] Bernard Fisher, Stewart Anderson, John Bryant, Richard G. Margolese, Melvin Deutsch, Edwin R. Fisher, Jong-Hyeon Jeong, and Norman Wolmark. Twenty-Year Follow-up of a Randomized Trial Comparing Total Mastectomy, Lumpectomy, and Lumpectomy plus Irradiation for the Treatment of Invasive Breast Cancer. New England Journal of Medicine, 347(16):1233-1241, October 2002.

[42] Bernard Fisher, Eleanor Montague, Carol Redmond, Bruce Barton, Donna Borland, Edwin R. Fisher, Melvin Deutsch, George Schwarz, Richard Margolese, William Donegan, Herbert Volk, Carl Konvolinka, Bernard Gardner, Isidore Cohn, Gerson Lesnick, Anatolio B. Cruz, Walter Lawrence, Thomas Nealon, Harvey Butcher, Richard Lawton, and Other Nsabp Investigators. Comparison of radical mastectomy with alternative treatments for primary breast cancer:A first report of results from a prospective randomized clinical trial. Cancer, 39(6):2827-2839, June 1977.

[43] Early Breast Cancer Trialists' Collaborative Group (EBCTCG). Effect of radiotherapy after breast-conserving surgery on 10-year recurrence and 15-year breast cancer death: metaanalysis of individual patient data for 10801 women in 17 randomised trials. The Lancet, 378(9804):1707-1716, November 2011.

[44] James D Yager. Estrogen Carcinogenesis in Breast Cancer. The New England Journal of Medicine, page 13, 2006.

[45] Gail M Wilkes and Margaret Barton-Burke. 2018 Oncology Nursing Drug Handbook. 2018. OCLC: 1030285714.

[46] Kathleen E. Grady, Jeanne P. Lemkau, Janine M. McVay, and Susan T. Reisine. The importance of physician encouragement in breast cancer screening of older women. Preventive Medicine, 21(6):766-780, November 1992.

[47] Robert A. Smith PhD and Suzanne Haynes PhD. Barriers to screening for breast cancer. page 11 .

[48] Umberto Veronesi, Natale Cascinelli, Luigi Mariani, Marco Greco, Roberto Saccozzi, Alberto Luini, Marisel Aguilar, and Ettore Marubini. Twenty-Year Follow-up of a Randomized Study Comparing Breast-Conserving Surgery with Radical Mastectomy for Early Breast Cancer. New England Journal of Medicine, 347(16):1227-1232, October 2002. 
[49] M.R. Christiaens, E. van der Schueren, and K. Vantongelen. More detailed documentation of operative procedures in breast conserving treatment: what good will it do us? European Journal of Surgical Oncology (EJSO), 22(4):326-330, August 1996.

[50] D.R.H. Christie, M.Y. O’Brien, J.A. Christie, T. Kron, S.A. Ferguson, C.S. Hamilton, and J.W. Denham. A comparison of methods of cosmetic assessment in breast conservation treatment. The Breast, 5(5):358-367, October 1996.

[51] Maria João Cardoso, Jaime Santos Cardoso, Conny Vrieling, Douglas Macmillan, Dick Rainsbury, Joerg Heil, Eric Hau, and Mohammed Keshtgar. Recommendations for the aesthetic evaluation of breast cancer conservative treatment. Breast Cancer Research and Treatment, 135(3):629-637, October 2012.

[52] F. Fitzal, W. Krois, H. Trischler, L. Wutzel, O. Riedl, U. Kühbelböck, B. Wintersteiner, M.J. Cardoso, P. Dubsky, M. Gnant, R. Jakesz, and T. Wild. The use of a breast symmetry index for objective evaluation of breast cosmesis. The Breast, 16(4):429-435, August 2007.

[53] Geert Litjens, Thijs Kooi, Babak Ehteshami Bejnordi, Arnaud Arindra Adiyoso Setio, Francesco Ciompi, Mohsen Ghafoorian, Jeroen A.W.M. van der Laak, Bram van Ginneken, and Clara I. Sánchez. A survey on deep learning in medical image analysis. Medical Image Analysis, 42:60-88, December 2017.

[54] K.S. Asgeirsson, T. Rasheed, S.J. McCulley, and R.D. Macmillan. Oncological and cosmetic outcomes of oncoplastic breast conserving surgery. European Journal of Surgical Oncology (EJSO), 31(8):817-823, October 2005.

[55] Navneet Kaur, Jean-Yves Petit, Mario Rietjens, Fausto Maffini, Alberto Luini, Giovanna Gatti, Pier Carlo Rey, Cicero Urban, and Francesca De Lorenzi. Comparative Study of Surgical Margins in Oncoplastic Surgery and Quadrantectomy in Breast Cancer. Annals of Surgical Oncology, 12(7):539-545, July 2005.

[56] E. Van Limbergen, E. van der Schueren, and K. Van Tongelen. Cosmetic evaluation of breast conserving treatment for mammary cancer. 1. Proposal of a quantitative scoring system. Radiotherapy and Oncology, 16(3):159-167, November 1989.

[57] Conny Vrieling, Laurence Collette, Alain Fourquet, Willem J Hoogenraad, Jean-Claude Horiot, Jos J Jager, Marianne Pierart, Philip M Poortmans, Henk Struikmans, Ben Maat, Erik Van Limbergen, and Harry Bartelink. The inuence of patient, tumor and treatment factors on the cosmetic results after breast-conserving therapy in the EORTC 'boost vs. no boost' trial. Radiotherapy and Oncology, page 14, 2000.

[58] Richard D. Pezner, Mary P. Patterson, L.Robert Hill, Nayana Vora, Kanta R. Desai, John O. Archambeau, and James A. Lipsett. Breast retraction assessment: an objective evaluation of cosmetic results of patients treated conservatively for breast cancer. International Journal of Radiation Oncology*Biology*Physics, 11(3):575-578, March 1985.

[59] Sk Al-Ghazal, Rw Blamey, J Stewart, and Aal Morgan. The cosmetic outcome in early breast cancer treated with breast conservation. European Journal of Surgical Oncology (EJSO), 25(6):566-570, December 1999.

[60] Scott A. Triedman, Robert Osteen, and Jay R. Harris. Factors Influencing Cosmetic Outcome of Conservative Surgery and Radiotherapy for Breast Cancer. Surgical Clinics of North America, 70(4):901-916, August 1990. 
[61] Richard D. Pezner, James A. Lipsett, Nayana L. Vora, and Kanta R. Desai. Limited usefulness of observer-based cosmesis scales employed to evaluate patients treated conservatively for breast cancer. International Journal of Radiation Oncology*Biology*Physics, 11(6):1117-1119, June 1985.

[62] Conny Vrieling, Laurence Collette, Ellen Bartelink, Jacques H. Borger, Stefan J. Brenninkmeyer, Jean-Claude Horiot, Marianne Pierart, Philip M. Poortmans, Henk Struikmans, Emmanuel Van der Schueren, Joop A. Van Dongen, Erik Van Limbergen, and Harry Bartelink. Validation of the methods of cosmetic assessment after breast-conserving therapy in the EORTC "boost versus no boost" trial. International Journal of Radiation Oncology*Biology*Physics, 45(3):667-676, October 1999.

[63] Sheila Stallard Caroline Eadie, Alex Herd. An investigation into digital imaging in assessing cosmetic outcome after breast surgery. Journal of Audiovisual Media in Medicine, 23(1):12-16, January 2000.

[64] Jay R. Harris, Martin B. Levene, Goran Svensson, and Samuel Hellman. Analysis of cosmetic results following primary radiation therapy for stages I and II carcinoma of the breast. International Journal of Radiation Oncology*Biology*Physics, 5(2):257-261, February 1979.

[65] D.V. Ash, E.A. Benson, J.R. Sainsbury, C. Round, and C. Head. Seven-year follow-up on 334 patients treated by breast conserving surgery and short course radical postoperative radiotherapy: A report of the Yorkshire breast cancer group. Clinical Oncology, 7(2):93-96, January 1995.

[66] Leela Krishnan, Annette L. Stanton, Charlotte A. Collins, Vici E. Liston, and William R. Jewell. Form or function? Part 2. Objective cosmetic and functional correlates of quality of life in women treated with breast-conserving surgical procedures and radiotherapy. Cancer, 91(12):2282-2287, June 2001.

[67] James R Gray. WOMEN: ANALYSISOFCOSMETICOUTCOME. page 1.

[68] Bajaj Ak, Kon Ps, and Oberg Kc. Aesthetic Outcomes in Patients Undergoing Breast Conservation Therapy for the Treatment of Localized Breast Cancer. page 1.

[69] Holli Kaija, Saaristo Rauni, Isola Jorma, and Hakama Matti. Consistency of patient- and doctor-assessed cosmetic outcome after conservative treatment of breast cancer. Breast Cancer Research and Treatment, 45(3):225-228, September 1997.

[70] Geoffrey F. Beadle, Steven Come, I.Craig Henderson, Barbara Silver, Samuel Hellman, and Jay R. Harris. The effect of adjuvant chemotherapy on the cosmetic results after primary radiation treatment for early stage breast cancer. International Journal of Radiation Oncology*Biology*Physics, 10(11):2131-2137, November 1984.

[71] Bernard Pierquin, Judith Huart, Michel Raynal, Yves Otmezguine, Elie Calitchi, JeanJacques Mazeron, Gérard Ganem, Jean-Paul Le Bourgeois, Ginette Marinello, Michel Julien, Bernard Brun, and Franck Feuilhade. Conservative treatment for breast cancer: long-term results (15 years). Radiotherapy and Oncology, 20(1):16-23, January 1991.

[72] Maria João Cardoso, Jaime Cardoso, Ana Cristina Santos, Henrique Barros, and Manuel Cardoso de Oliveira. Interobserver agreement and consensus over the esthetic evaluation of conservative treatment for breast cancer. The Breast, 15(1):52-57, February 2006. 
[73] L. I. Tsouskas and I. S. Fentiman. Breast compliance: A new method for evaluation of cosmetic outcome after conservative treatment of early breast cancer. Breast Cancer Research and Treatment, 15(3):185-190, May 1990.

[74] M. Noguchi, Y. Saito, Y. Mizukami, A. Nonomura, N. Ohta, N. Koyasaki, T. Taniya, and I. Miyazaki. Breast deformity, its correction, and assessment of breast conserving surgery. Breast Cancer Research and Treatment, 18(2):111-118, May 1991.

[75] Jaime S. Cardoso and Maria J. Cardoso. Breast Contour Detection for the Aesthetic Evaluation of Breast Cancer Conservative Treatment. In Janusz Kacprzyk, Marek Kurzynski, Edward Puchala, Michal Wozniak, and Andrzej Zolnierek, editors, Computer Recognition Systems 2, volume 45, pages 518-525. Springer Berlin Heidelberg, Berlin, Heidelberg, 2007.

[76] H. Mirzaalian, M.R. Ahmadzadeh, and F. Kolahdoozan. Breast Contour Detection on Digital Mammogram. In 2006 2nd International Conference on Information \& Communication Technologies, volume 1, pages 1804-1808, Damascus, Syria, 2006. IEEE.

[77] Ricardo Sousa, Jaime S. Cardoso, J. F. Pinto da Costa, and M. J. Cardoso. Breast contour detection with shape priors. In 2008 15th IEEE International Conference on Image Processing, pages 1440-1443, San Diego, CA, USA, 2008. IEEE.

[78] Juhun Lee, G. S. Muralidhar, G. P. Reece, and M. K. Markey. A shape constrained parametric active contour model for breast contour detection. In 2012 Annual International Conference of the IEEE Engineering in Medicine and Biology Society, pages 4450-4453, San Diego, CA, August 2012. IEEE.

[79] Juhun Lee, Si Chen, G. P. Reece, M. A. Crosby, E. K. Beahm, and M. K. Markey. A Novel Quantitative Measure of Breast Curvature Based on Catenary. IEEE Transactions on Biomedical Engineering, 59(4):1115-1124, April 2012.

[80] Robert C Yates. Curves and Their Properties. 1974.

[81] John Oprea. Differential geometry and its applications. MAA, 2007.

[82] Cardoso et al. AUTOMATIC BREAST CONTOUR DETECTION IN DIGITAL PHOTOGRAPHS:. In Proceedings of the First International Conference on Health Informatics, pages 91-98, Funchal, Madeira, Portugal, 2008. SciTePress - Science and and Technology Publications.

[83] Nitin Udpa, Mehul P. Sampat, Min Soon Kim, Gregory P. Reece, and Mia K. Markey. Objective assessment of the aesthetic outcomes of breast cancer treatment: toward automatic localization of fiducial points on digital photographs. page 651420, San Diego, CA, March 2007.

[84] Dewi Nasien, Siti S. Yuhaniz, and Habibollah Haron. Statistical Learning Theory and Support Vector Machines. In 2010 Second International Conference on Computer Research and Development, pages 760-764, Kuala Lumpur, Malaysia, 2010. IEEE.

[85] Jaime S. Cardoso, Inês Domingues, and Hélder P. Oliveira. Closed Shortest Path in the Original Coordinates with an Application to Breast Cancer. International Journal of Pattern Recognition and Artificial Intelligence, 29(01):1555002, February 2015. 
[86] Zhe Cao, Tomas Simon, Shih-En Wei, and Yaser Sheikh. Realtime Multi-Person 2d Pose Estimation using Part Affinity Fields. arXiv:1611.08050 [cs], November 2016. arXiv: 1611.08050.

[87] Vasileios Belagiannis and Andrew Zisserman. Recurrent Human Pose Estimation. arXiv:1605.02914 [cs], May 2016. arXiv: 1605.02914.

[88] Karen Simonyan and Andrew Zisserman. Very Deep Convolutional Networks for LargeScale Image Recognition. arXiv:1409.1556 [cs], September 2014. arXiv: 1409.1556.

[89] E. W. Dijkstra. A note on two problems in connexion with graphs. Numerische Mathematik, 1(1):269-271, December 1959.

[90] Rafael C. Gonzalez and Richard E. Woods. Digital image processing. Prentice Hall, Upper Saddle River, N.J, 3rd ed edition, 2008.

[91] Neeraj Sharma, AmitK Ray, Kk Shukla, Shiru Sharma, Satyajit Pradhan, Arvind Srivastva, and LalitM Aggarwal. Automated medical image segmentation techniques. Journal of Medical Physics, 35(1):3, 2010.

[92] Maedeh Sadat Fasihi and Wasfy B. Mikhael. Overview of Current Biomedical Image Segmentation Methods. In 2016 International Conference on Computational Science and Computational Intelligence (CSCI), pages 803-808, Las Vegas, NV, USA, December 2016. IEEE.

[93] Nikhilr Pal, Sankark Pal, and Machine IntelligenceUnit. A REVIEW ON IMAGE SEGMENTATION TECHNIQUES. page 18.

[94] P.K. Saha and J.K. Udupa. Optimum image thresholding via class uncertainty and region homogeneity. IEEE Transactions on Pattern Analysis and Machine Intelligence, 23(7):689706, July 2001.

[95] Nobuyuki Otsu. A Threshold Selection Method from Gray-Level Histograms. IEEE Transactions on Systems, Man, and Cybernetics, 9(1):62-66, January 1979.

[96] Dr H B Kekre and Saylee M Gharge. Image Segmentation using Extended Edge Operator for Mammographic Images. 02(04):6, 2010.

[97] E. A. Zanaty. Improved Region Growing Method for Magnetic Resonance Images (MRIs) Segmentation. American Journal of Remote Sensing, 1(2):53, 2013.

[98] Juan Shan, H.D. Cheng, and Yuxuan Wang. A completely automatic segmentation method for breast ultrasound images using region growing. In Proceedings of the 11th Joint Conference on Information Sciences (JCIS), The Harbin Institue of Technology, Shenzhen, China, 2008. Atlantis Press.

[99] P R Tamilselvi and Dr P Thangaraj. Segmentation of Calculi from Ultrasound Kidney Images by Region Indicator with Contour Segmentation Method. page 10, 2011.

[100] Ellen Day, James Betler, David Parda, Bodo Reitz, Alexander Kirichenko, Seyed Mohammadi, and Moyed Miften. A region growing method for tumor volume segmentation on PET images for rectal and anal cancer patients: A region growing method for tumor segmentation. Medical Physics, 36(10):4349-4358, September 2009. 
[101] J. Bernard Davis, Beatrice Reiner, Marius Huser, Cyrill Burger, Gábor Székely, and I. Frank Ciernik. Assessment of $18 \mathrm{f}$ PET signals for automatic target volume definition in radiotherapy treatment planning. Radiotherapy and Oncology, 80(1):43-50, July 2006.

[102] Michael Kass, Andrew Witkin, and Demetri Terzopoulos. Snakes: Active contour models. International Journal of Computer Vision, 1(4):321-331, January 1988.

[103] Chenyang Xu and J.L. Prince. Snakes, shapes, and gradient vector flow. IEEE Transactions on Image Processing, 7(3):359-369, March 1998.

[104] Viranee Thongnuch and Bunyarit Uyyanonvara. AUTOMATIC OPTIC DISK DETECTION FROM LOW CONTRAST RETINAL IMAGES OF ROP INFANT USING GVF SNAKE. page 13.

[105] Yuri Boykov and Gareth Funka-Lea. Graph Cuts and Efficient N-D Image Segmentation. International Journal of Computer Vision, 70(2):109-131, November 2006.

[106] Alex Krizhevsky, Ilya Sutskever, and Geoffrey E. Hinton. ImageNet classification with deep convolutional neural networks. Communications of the ACM, 60(6):84-90, May 2017.

[107] Christian Szegedy, Wei Liu, Yangqing Jia, Pierre Sermanet, Scott Reed, Dragomir Anguelov, Dumitru Erhan, Vincent Vanhoucke, and Andrew Rabinovich. Going Deeper with Convolutions. arXiv:1409.4842 [cs], September 2014. arXiv: 1409.4842.

[108] Pierre Sermanet, David Eigen, Xiang Zhang, Michael Mathieu, Rob Fergus, and Yann LeCun. OverFeat: Integrated Recognition, Localization and Detection using Convolutional Networks. arXiv:1312.6229 [cs], December 2013. arXiv: 1312.6229.

[109] Ross Girshick, Jeff Donahue, Trevor Darrell, and Jitendra Malik. Rich feature hierarchies for accurate object detection and semantic segmentation. arXiv:1311.2524 [cs], November 2013. arXiv: 1311.2524.

[110] Kaiming He, Xiangyu Zhang, Shaoqing Ren, and Jian Sun. Spatial Pyramid Pooling in Deep Convolutional Networks for Visual Recognition. arXiv:1406.4729 [cs], 8691:346361, 2014. arXiv: 1406.4729.

[111] Jonathan Long, Ning Zhang, and Trevor Darrell. Do Convnets Learn Correspondence? arXiv:1411.1091 [cs], November 2014. arXiv: 1411.1091.

[112] Ning Zhang, Jeff Donahue, Ross Girshick, and Trevor Darrell. Part-based R-CNNs for Fine-grained Category Detection. arXiv:1407.3867 [cs], July 2014. arXiv: 1407.3867.

[113] Philipp Fischer, Alexey Dosovitskiy, and Thomas Brox. Descriptor Matching with Convolutional Neural Networks: a Comparison to SIFT. arXiv:1405.5769 [cs], May 2014. arXiv: 1405.5769 .

[114] Y. LeCun, B. Boser, J. S. Denker, D. Henderson, R. E. Howard, W. Hubbard, and L. D. Jackel. Backpropagation Applied to Handwritten Zip Code Recognition. Neural Computation, 1(4):541-551, December 1989.

[115] Dan C Ciresan, Luca M Gambardella, and Alessandro Giusti. Deep Neural Networks Segment Neuronal Membranes in Electron Microscopy Images. page 9. 
[116] Alex Krizhevsky, Ilya Sutskever, and Geoffrey E. Hinton. ImageNet classification with deep convolutional neural networks. Communications of the ACM, 60(6):84-90, May 2017.

[117] Karen Simonyan and Andrew Zisserman. Very Deep Convolutional Networks for LargeScale Image Recognition. arXiv:1409.1556 [cs], September 2014. arXiv: 1409.1556.

[118] Christian Szegedy, Vincent Vanhoucke, Sergey Ioffe, Jonathon Shlens, and Zbigniew Wojna. Rethinking the Inception Architecture for Computer Vision. arXiv:1512.00567 [cs], December 2015. arXiv: 1512.00567.

[119] Kaiming He, Xiangyu Zhang, Shaoqing Ren, and Jian Sun. Deep Residual Learning for Image Recognition. arXiv:1512.03385 [cs], December 2015. arXiv: 1512.03385.

[120] Vijay Badrinarayanan, Alex Kendall, and Roberto Cipolla. SegNet: A Deep Convolutional Encoder-Decoder Architecture for Image Segmentation. arXiv:1511.00561 [cs], November 2015. arXiv: 1511.00561.

[121] Hyeonwoo Noh, Seunghoon Hong, and Bohyung Han. Learning Deconvolution Network for Semantic Segmentation. In 2015 IEEE International Conference on Computer Vision (ICCV), pages 1520-1528, Santiago, Chile, December 2015. IEEE.

[122] Liang-Chieh Chen, George Papandreou, Iasonas Kokkinos, Kevin Murphy, and Alan L. Yuille. Semantic Image Segmentation with Deep Convolutional Nets and Fully Connected CRFs. arXiv:1412.7062 [cs], December 2014. arXiv: 1412.7062.

[123] Fisher Yu and Vladlen Koltun. Multi-Scale Context Aggregation by Dilated Convolutions. arXiv:1511.07122 [cs], November 2015. arXiv: 1511.07122.

[124] Saining Xie and Zhuowen Tu. Holistically-Nested Edge Detection. arXiv:1504.06375 [cs], April 2015. arXiv: 1504.06375.

[125] Liang-Chieh Chen, George Papandreou, Florian Schroff, and Hartwig Adam. Rethinking Atrous Convolution for Semantic Image Segmentation. arXiv:1706.05587 [cs], June 2017. arXiv: 1706.05587.

[126] Liang-Chieh Chen, George Papandreou, Iasonas Kokkinos, Kevin Murphy, and Alan L. Yuille. Semantic Image Segmentation with Deep Convolutional Nets and Fully Connected CRFs. arXiv:1412.7062 [cs], December 2014. arXiv: 1412.7062.

[127] Liang-Chieh Chen, George Papandreou, Iasonas Kokkinos, Kevin Murphy, and Alan L. Yuille. DeepLab: Semantic Image Segmentation with Deep Convolutional Nets, Atrous Convolution, and Fully Connected CRFs. arXiv:1606.00915 [cs], June 2016. arXiv: 1606.00915 .

[128] Wei Liu, Andrew Rabinovich, and Alexander C. Berg. ParseNet: Looking Wider to See Better. arXiv:1506.04579 [cs], June 2015. arXiv: 1506.04579.

[129] Hengshuang Zhao, Jianping Shi, Xiaojuan Qi, Xiaogang Wang, and Jiaya Jia. Pyramid Scene Parsing Network. arXiv:1612.01105 [cs], December 2016. arXiv: 1612.01105.

[130] Vijay Badrinarayanan, Alex Kendall, and Roberto Cipolla. SegNet: A Deep Convolutional Encoder-Decoder Architecture for Image Segmentation. arXiv:1511.00561 [cs], November 2015. arXiv: 1511.00561. 
[131] Olaf Ronneberger, Philipp Fischer, and Thomas Brox. U-Net: Convolutional Networks for Biomedical Image Segmentation. arXiv:1505.04597 [cs], May 2015. arXiv: 1505.04597.

[132] François Chollet. Xception: Deep Learning with Depthwise Separable Convolutions. arXiv:1610.02357 [cs], October 2016. arXiv: 1610.02357.

[133] Fausto Milletari, Nassir Navab, and Seyed-Ahmad Ahmadi. V-Net: Fully Convolutional Neural Networks for Volumetric Medical Image Segmentation. arXiv:1606.04797 [cs], June 2016. arXiv: 1606.04797.

[134] François Chollet and others. Keras, 2015.

[135] Adam Paszke, Sam Gross, Soumith Chintala, Gregory Chanan, Edward Yang, Zachary DeVito, Zeming Lin, Alban Desmaison, Luca Antiga, and Adam Lerer. Automatic differentiation in PyTorch. 2017.

[136] Matthew D. Zeiler. ADADELTA: An Adaptive Learning Rate Method. arXiv:1212.5701 [cs], December 2012. arXiv: 1212.5701.

[137] Peter C Fishbumr. UTILITY THEORY FOR DECISION MAKING. page 246.

[138] Kelwin Fernandes, Jaime S. Cardoso, and Hector Palacios. Learning and ensembling lexicographic preference trees with multiple kernels. In 2016 International Joint Conference on Neural Networks (IJCNN), pages 2140-2147, Vancouver, BC, Canada, July 2016. IEEE.

[139] D. Ellsberg. Classic and Current Notions of "Measurable Utility". The Economic Journal, 64(255):528, September 1954.

[140] Ann C Séror. Internet Infrastructures and Health Care Systems: a Qualitative Comparative Analysis on Networks and Markets in the British National Health Service and Kaiser Permanente. Journal of Medical Internet Research, 4(3):e21, December 2002.

[141] F. Ricca and P. Tonella. Analysis and testing of Web applications. In Proceedings of the 23rd International Conference on Software Engineering. ICSE 2001, pages 25-34, Toronto, Ont., Canada, 2001. IEEE Comput. Soc.

[142] Jiang Wang, Yi Yang, Junhua Mao, Zhiheng Huang, Chang Huang, and Wei Xu. CNNRNN: A Unified Framework for Multi-label Image Classification. In 2016 IEEE Conference on Computer Vision and Pattern Recognition (CVPR), pages 2285-2294, Las Vegas, NV, USA, June 2016. IEEE.

[143] Peter W. Battaglia, Jessica B. Hamrick, Victor Bapst, Alvaro Sanchez-Gonzalez, Vinicius Zambaldi, Mateusz Malinowski, Andrea Tacchetti, David Raposo, Adam Santoro, Ryan Faulkner, Caglar Gulcehre, Francis Song, Andrew Ballard, Justin Gilmer, George Dahl, Ashish Vaswani, Kelsey Allen, Charles Nash, Victoria Langston, Chris Dyer, Nicolas Heess, Daan Wierstra, Pushmeet Kohli, Matt Botvinick, Oriol Vinyals, Yujia Li, and Razvan Pascanu. Relational inductive biases, deep learning, and graph networks. arXiv:1806.01261 [cs, stat], June 2018. arXiv: 1806.01261. 